\title{
PHOTOPRODUCTION OF CHARMED BARYONS
}

\section{BY}

JAMES JOHN RUSSELL

B.S., Ohio State University, 1972

M.S., University of Illinois, 1973

\section{THESIS}

Submitted in partial fulfillment of the requirements for the degree of Doctor of Philosophy in Physics in the Graduate College of the University of Illinois at Urbana-Champaign, 1980 


\title{
PHOTOPRODUCTION OF CHARMED BAKYONS
}

\author{
James John Russe11, Ph.D. \\ Department of Physics \\ University of Illinois at Urbana-Champaign, 1980
}

The results of a search for the photoproduction of charmed baryons in the broad-band neutral beam at Fermi National Accelerator Laboratory are reported. The lowest lying charmed baryon $\left(\Lambda_{c}^{+}\right)$is observed through its decay to $p-\bar{K}^{0}$. The cross section times branching ratio of

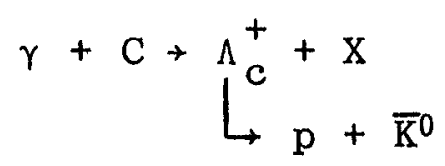

is measured to be $\sigma B=3$ nanobarns/nucleon. The total error on this measurement is estimated to be $-20 \%$ to $+40 \%$. The mass of the $\Lambda_{\mathrm{c}}^{+}$is found to be $2.284 \pm 0.001 \mathrm{GeV} / \mathrm{c}^{2}$, in good agreement with the Mark II result from SPEAR. Upper limits $(90 \%$ confidence level) are set on $\sigma B$ for the modes $\Lambda^{0} \pi, \Lambda^{0} \pi \pi \pi, \mathrm{pK} \pi$. 


\section{ACKNOWLEDGEMENTS}

Throughout this work I have tried not only to give the facts but also the flavor of the experiment. No such description would be complete without mentioning the people involved. Good physics can only be done by people who have an enthusiasm and love for it. Being surrounded by people who share these feelings only serves to reinforce them. Each member of our group has become a lasting friend, and I would like to take this opportunity to thank each individually:

To our technical help, Leonard D. Seward from the University of Illinois and Ken Gray of Columbia University who did so much more than they were called upon to do;

To Maged Atiya, Steve Holmes, and Bill Wisniewski for their hard work, particularly in the early phases of the experiment;

To Wonyong Lee our irrepressible leader;

To fellow Illinois members Paul Avery, Gary Gladding, Maury Goodman, Tom O'Halloran, Al Wattenberg, and James Wiss for their help in the analysis and throughout the experiment;

To Morris Binkley for bearing the onus of keeping the cryostat and chambers in operating condition by one method or another ;

And to John Peoples, whose unbounded enthusiasm tempered by practical reality and supported with hard work, served as a continual source of new ideas.

I would like to give a special thanks to my advisor, Michael Gormley, who has provided the steadying influence needed to smooth out the emotional peaks and valleys which are bound to 
occur during any enterprise lasting seven years. A special thanks is also reserved for Joel Butler, John Cumalat, and Irwin Gaines. The sadness I feel when I leave Fermilab will be for their lost company. Three greater friends one could not hope to find. Without the support provided by these three, I would have given up long ago.

I wish to dedicate this work to my parents and my wife, Judy. Parents have a way of saying, "Someday you'll thank me for this." This is that day, and this is my thanks to them. I hope I will always be a positive reflection on them. Finally to my wife, who has endured these seven years of graduate school, my special thanks. It would not have been possible without her love.

This research was supported in part by the U. S. Department of Energy under Contract EY-76-C-02-1195. 
TABLE OF CONTENTS

Page

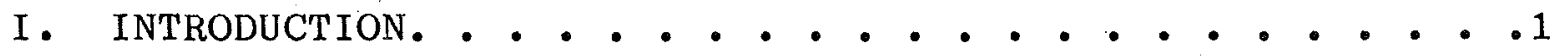

A. Review of Charm: Theoretical . . . . . . . . 1

B. Review of Charm: Experimental. . . . . . . . 3

I . EXPERIMENTAL DESIGN • • • • • • • • • • • • • . 7

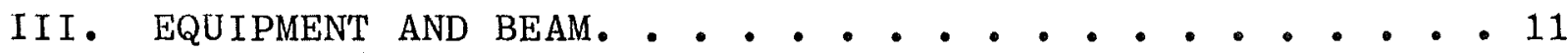

A. The Beam. • . . . . . . . . . . . . . 11

B. Charged Track Detection . . . . . . . . . 15

C. Cherenkov Counters. . . . . . . . . . . . 18

D. Other Features. . . . . . . . . . . . . . 22

E. Data Acquisition and Triggering . . . . . . . 26

IV. DATA ANALYSIS • • • • • • • • • • • • • • • 37

A. Reconstruction. . . . . . . . . . . . . 38

B. Higher Level Analysis . . . . . . . . . . . 41

1. Momenta and Vertex Determination. . . . . 41

2. Vo Identification . . . . . . . . . 46

3. Cherenkov Identification. . . . . . . . 52

C. Event Selection . . . . . . . . . . . . 58

V CHARM BARYON SEARCH . . . . . . . . . . . . . 60

A. $\Lambda^{0} \pi$ and $\Lambda^{0} \pi \pi \pi$. . . . . . . . . . . . 60

B. $\mathrm{pK \pi} \cdot$. . . . . . . . . . . . . . . 70

C. $\mathrm{pK}_{\mathrm{S}}^{0}$. . . . . . . . . . . . . . . . 70

D. Monte Carlo . . . . . . . . . . . . 83

E. Cross Sections and Conclusions. . . . . . . . 88

VI. CONCLUSIONS • • • • • • • • • • • • • • • • • 89

A. Comments on the Mass of the $\Lambda_{c}{ }^{+} \cdot$. . . . . . 89 


\section{TABLE OF CONTENTS}

Page

B. Comments on the Charm Cross Section . . . . . . 90

C. Comments on the Future of Charm Experiments . . . 91 LIST OF REFERENCES. • • • • • • • • . • • • • • • 93 APPENDIX A. . . . . . . . . . . . . . . . . . . . 95 APPENDIX B. . . . . . . . . . . . . . . . . . . . 100 APPENDIX C. • . . . . . . . . • • . . . . . . .104

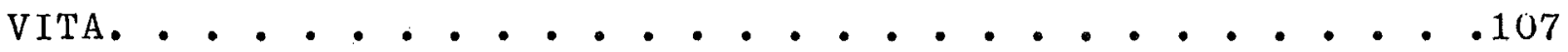




\section{INTRODUCTION}

\section{A. Keview of Charm: Theoretical}

The need to break the complex facets of nature into small comprehensible blocks is as old as science, dating back to the first classification scheme of earth, wind, fire, and water. The underlying principle of these efforts has been a commitment to the idea the blocks be small in number and display some internal symmetry. These notations, while more philosophical than anything else, nevertheless have provided a useful and productive working framework.

Particle physics may be the best example of these ideas in action, where new schemes periodically succeed older ones which have outgrown their original elegance. In 1948, Fermi and Yangl attempted to reduce the growing number of "elementary particles" by postulating that mesons were actually nucleon-antinucleon pairs. The simple isospin assignments were

$$
\mathrm{P}=|1 / 2,1 / 2\rangle \quad \mathrm{N}=|-1 / 2,1 / 2\rangle \text {. }
$$

Adding the isospins gives an isotriplet identified as $\pi^{-}, \pi^{0}, \pi^{+}$ accounting for the then known mesons, and an isosinglet, the $n^{0}$.

This model was adequate until the discovery of strangeness. Various models were given by Sakata ${ }^{2}$ and Gell-Mann and $\mathrm{Ne}^{1} \mathrm{eman}^{3}, 4$ which finally evolved to the three-quark model in which known particles are thought of as combinations of more fundamental blocks called quarks; the mesons being a quarkantiquark pair and the baryons being three quarks. The quantum numbers assigned to the three quarks are 


$\begin{array}{ccccccc} & \underline{\mathrm{I}} & \underline{\mathrm{I}} & \underline{\mathrm{Q}} & \underline{\mathrm{S}} & \underline{\mathrm{B}} & \underline{\mathrm{Y}} \\ \mathrm{u} & 1 / 2 & +1 / 2 & 2 / 3 & 0 & 1 / 3 & 1 / 3 \\ \underline{\mathrm{d}} & 1 / 2 & -1 / 2 & -1 / 3 & 0 & 1 / 3 & 1 / 3 \\ \underline{\mathrm{S}} & 0 & 0 & -1 / 3 & -1 & 1 / 3 & -2 / 3\end{array}$

The quark model illustrates a universal, although not necessary, feature of a successful model, the ability to make predictions which are experimentally verifiable. Thus it is nore than a collection of ad hoc rules whose only contribution is succintness. In addition to accommodating all known particles, the initial quark model also predicted the existence of a hitherto unknown particle, the $\Omega^{-}$. The subsequent discovery of a particle $e^{5}$ with the predicted properties lent credibility to the quark model.

The three-quark model was not without its faults. Predicted rates for $\mathrm{K} \rightarrow \mu^{+}{ }_{\mu}$ and $\mathrm{K}^{+} \rightarrow \pi^{+} \nu \bar{\nu}$ were not observed. ${ }^{6}$ The model could not explain the $K_{L}^{0}-K_{S}^{0}$ mass difference. All these processes involve a coupling between the $s$ and $d$ quarks, a neutral strangeness changing hadronic current, which was allowed by the model but experimentally did not exist.

The basic idea of the model was preserved and these questions addressed, when Bjorken, Glashow, Iliopoulos, and Miani ${ }^{7,8}$ added a fourth quark with a new quantum number called charm. Later Weinberg ${ }^{9}$ and Salam $^{10}$ used this quark to simultaneously suppress the neutral strangeness changing currents and achieve a lepton-hadron symmetry (see Appendix B). The quantum numbers of the charm quark are
I $\quad \underline{\mathrm{I}}$
$\underline{Q}$
$\underline{\mathrm{S}}$
$\underline{\mathrm{B}}$
$\underline{\mathrm{X}}$
$\underline{c}$
0
$2 / 3$
0
$1 / 3$
$1 / 3$ 
While the model did accomplish its initial goals, it made the spectacular prediction of a complete new SU(3) multiplet of baryons and mesons (see Appendix A). Using the known $K_{L}^{0}-K_{S}^{0}$ mass difference and $\mathrm{K}^{0}+\mu^{+}{ }^{-}$and $\mathrm{K}^{+} \rightarrow \pi^{+} \nu \bar{\nu}$ one could get a rough estimate of the new quark's mass of less than several $\mathrm{GeV} / \mathrm{c}^{2}$, thus setting the mass scale of the charmed particles. Quite remarkably, there were no known particles which were candidates for charm.

The theorists had clearly done all that could be expected, produce a theory which accommodated all known facts, displayed a simple internal symmetry, and made experimentally verifiable predictions. The job of the experimenters was clear and seemingly simple: find these new states.

\section{B. Review of Charm: Experimental}

The early attempts to observe charm particles served as a forboding of the future. Even though the properties of charm were well defined, success was difficult. The discovery of the $\psi$ in November $1974^{11,12}$ and other family members ${ }^{3}$ shortly thereafter, established the viability of the charm model. With a mass of $3.1 \mathrm{GeV} / \mathrm{c}^{2}$ and a width of $6.7 \mathrm{KeV} / \mathrm{c}^{2}$ the $\psi$ was a prime candidate for the $c \bar{c}$ meson. The $\psi$ has only latent charm so that, while unlikely, alternative explanations were still being entertained. Clearly the prize trophy was a particle exhibiting explicit charm.

The properties of the $\psi$ and its family tightened the predictions on charm-particle properties. The narrow width of the $\psi$ is interpreted in terms of an empirical mechanism called 
Zweig's Rule.14-16 Zweig's Rule is often invoked to explain the dominance of the KK decay of the $\Phi$ over the $\pi^{+} \pi^{-} \pi^{0}$ decay. The preferred decay mode of the $\psi$ should be to two charmed mesons, analogous to the $\Phi^{\prime}$ 's decay to two strange mesons. If the $\psi^{\prime} s$ mass were below the kinematic limit of this reaction, then, according to Zweig's Rule, it cannot decay strongly. This information plus the observation that the next member of the $\psi$ family above the $\psi^{\prime}\left(3.675 \mathrm{GeV} / \mathrm{c}^{2}\right)$ at $4.1 \mathrm{GeV} / \mathrm{c}^{2}$ is wide and, therefore, is presumably above the charm threshold, puts a stringent limit on the mass of the charmed meson. It can be no less than half $3.675 \mathrm{GeV} / \mathrm{c}^{2}$ and no more than half $4.1 \mathrm{GeV} / \mathrm{c}^{2}$. This agrees well with the naive guess which gives the mass as the sum of the masses of its constituent quarks. The lowest lying charmed meson should be the isodoublet $\left(D^{0}, D^{+}\right)$with quark content $(c \bar{u}, c \bar{d})$. Taking the mass of the charmed quark to be $1.55 \mathrm{GeV} / \mathrm{c}^{2}$ (half the mass of the $\psi$ ) and the $u, d$ quarks to be $0.300 \mathrm{GeV} / \mathrm{c}^{2}$ one gets the same prediction.

In 1976 SPEAR reported the observation of two narrow states, one decaying into $\mathrm{K}^{\mp} \pm, \mathrm{K}_{\mathrm{S}^{0}}^{0}{ }^{+} \pi^{-}, \mathrm{K}_{\pi}^{\mp} \pm_{\pi}^{+} \pi^{-}$, and the other decaying $\mathrm{K}_{\pi}^{\mp} \pm_{\pi} \pm .17$ Equaliy important was the non-observation of the decay modes which do not have the charge-strangeness correlation predicted by the model. Other properties were in good agreement:

1. The masses were $1.863 \mathrm{GeV} / \mathrm{c}^{2}$ for the $\mathrm{D}^{0}$ and $1.868 \mathrm{GeV} / \mathrm{c}^{2}$ for the $\mathrm{D}^{+}$. Both had widths experimentally consistent with. zero.

2. There was some evidence for the analogous vector states of these pseudoscalars at about $2.01 \mathrm{GeV} / \mathrm{c}^{2}$.

3. The rise in $\mathrm{R}=\sigma\left(\mathrm{e}^{+} \mathrm{e}^{-} \rightarrow\right.$ hadrons $) / \sigma\left(\mathrm{e}^{+} \mathrm{e}^{-} \rightarrow \mu^{+} \mu^{-}\right)$ coincided correctly in both magnitude and energy with the charm threshold. 
In 1977 the only direct evidence of charm was from the $\mathrm{e}^{+} \mathrm{e}^{-}$ storage rings at SPEAR. Only indirect evidence through the number of direct leptons or neutrinos produced in association with strange particles was seen in hadron production.18-20 In addition to completing the charm spectroscopy, verification of the $\mathrm{e}^{+} \mathrm{e}^{-}$results was needed. Branching ratios to various final states and photoproduction cross sections, which can be related to hadron cross sections, were also important measurements. The direct observation of charmed baryons was of particular importance. Oniy two observations, neither of which was entirely convincing, had been reported.21,22 The lowest lying charm baryon, predicted to be the $c(u d)_{a}$ [where (ud) a means the antisymmetric arrangement of the $u$ and $d$ quarks] is stable against strong decays if its mass is below the sum of the proton and charm meson mass. Given that the mass predicted by adding the quark masses $\left(2.1-2.3 \mathrm{GeV} / \mathrm{c}^{2}\right)$ is far below the $\mathrm{pD}^{0}$ threshold (2.8 $\left.\mathrm{GeV} / \mathrm{c}^{2}\right)$, the charmed baryons can only decay weakly, and, therefore, will appear as a narrow resonance in an invariant mass distribution of its decay products.

To answer these questions a more sensitive, secondgeneration experiment was necessary. The design of such an experiment was begun in spring of 1977. The goals of the experiment (in order of priority) are listed below.

1. To establish conclusively the existence of the lowest lying. charmed baryon and anti-baryon by direct observation.

2. To determine the cross section for the hadronic production of charmed baryons. 
3. To establish as many decay modes of the charmed baryon as possible and measure their branching ratios.

4. To measure the cross section for the hadronic production of charmed mesons.

5. To search for excited states of the charmed baryon and for the lowest lying charmed strange meson $\left(F^{+}\right)$.

The choice of detector and its design requirenents for an experiment with these objectives are described in detail in Chapters II and III. The data analysis and interpretation of the results, discussed in Chapters IV and $V$, demonstrate that the first three goals were completed successfully. The fourth major goal was also successful but is the subject of a separate publication. ${ }^{3}$ The result of the fifth goal was, at best, inconclusive. 
II. EXPERIMENTAL DESIGN

The most convincing evidence for charm comes from the $\mathrm{e}^{+} \mathrm{e}^{-}$ machines.24 This seems paradoxical given that these cross sections are lower than the predictions for either hadron or photon beams. The hadronic searches for charm are plagued by high multiplicities in the final state with the charmed particles emerging at low Feynman $X$ ( $E$ of particle/E of beam) making detection in a conventional forward spectrometer difficult. In $\mathrm{e}^{+} \mathrm{e}^{-}$ beams the events are exclusive charm production eliminating the need to search through the many tracks found in a typical hadronic event to find the charm-decay products.

A charm search in a photon beam should be an effective compromise between the cleanliness of $\mathrm{e}^{+} \mathrm{e}^{-}$production and the potential rates in a hadron beam. The observation of the $\psi$ in photoproduction 25,26 establishes that charm does indeed couple to the photon. Using the $\psi$-photoproduction cross section, the $\psi$ nucleon cross section, ${ }^{26}$ and a vector dominance model, one gets a prediction of 1-2 $\mu b^{27}$ for the total charm cross section. The photon's total hadronic cross section is $110 \mu \mathrm{b}^{28}$ so on the surface it appears $1 \%$ of the hadronic rate is charm-associated.

This line of logic points directly to the heart of the problems with experimental observation of charmed states. Endless factors of 2,5 , and 10 compound to decrease this 1-2 $\mu \mathrm{b}$ to observable processes that have cross sections in nanobarns not microbarns. The biggest price one pays is that one does not observe "charm" but a specific final state. The 1-2 $\mu$ b estimate represents the production of all charmed particles and is shared 
in an unknown way between the charmed baryons, D's, D*'s, F's, $F^{2}$ 's, etc. What initially looked like $1-2 \mu \mathrm{b}$ is at most only 0.2-0.5 $\mathrm{\mu b}$ for any specific charmed particle. Given the large mass, charm also has many decay modes accessible to it even with the restriction that favored modes, include a strange particle. Many of these modes are either experimentally difficult, such as those involving one or more $\pi^{0}$ 's, or virtually impossible, such as those including $\mathrm{K}_{\mathrm{L}}^{0}$ 's, neutrons, or neutrinos, so that finding one with a branching ratio of more than $5 \%$ is rare.

This then is the number the experimentalist starts with. The potential observable rate is $5 \%$ of $0.5 \mu \mathrm{b}$, just $25 \mathrm{nb}$, representing $0.025 \%$ of the hadronic photon rate. Up to this point one has been at the mercy of physics. The experimenter still has the task of detecting, collecting, measuring, and identifying the final-state properties. The number of measurements needed to see a charm particle decay is large. Even if the inefficiencies involved in making these measurements were small, their sheer numbers add up and eventually take their toll so that a good detector would still be pressed to achieve $50 \%$ efficiency in a particular final state. Often overlooked, but nevertheless equally important, is the requirement that the experiment be accomplished with finite time and money. This condition makes final-state detection efficiencies of the order of $5-10 \%$ more typical.

The above is only one side of a double-edged knife. The other side is background. The amount of signal can usually be estimated, if not with great reliability, then at least with 
great conviction (read hope). Backgrounds are, for the most part, unknown until encountered.

To a high degree only limited control can be exercised over backgrounds. As previously alluded to, the selection of the beam particle helps. Certainly more information about the final state increases the chances of understanding the precise nature of the event. The narrow widths of charmed particles offer another way to cope with the backgrounds. The background is smoothly distributed over the entire mass region, whereas the signal will occur at only one mass. Increasing the mass resolution allows one to spread the background thinner while leaving the signal untouched. This is a particularly attractive solution. As opposed to most other methods of dealing with the background, increasing the mass resolution does not involve the usual penalty in signal detected.

The realities of the anticipated small cross sections and formidable backgrounds impose the following requirements on the design of an experiment whose goal is to directly observe charm by a peak in the invariant mass distribution of its decay products.

1. A large - aperture forward spectrometer is needed. The spectrometer should be finely granulated in order to cope with the anticipated complex topologies of charm events and to provide the precision measurements needed for achieving the necessary mass resolution.

2. As seen in Appendix B, the Cabbibo favored decays of charmed particles usually involve strange particles. The decay of charmed baryons have the additional requirement of containing a baryon in the final state. While the magnetic spectrometer can provide some particle identification ability by recognizing the charge particle decay modes of $\Lambda^{0}$ 's and $\mathrm{K}_{\mathrm{S}}^{0} \mathrm{~s}$, more is 
required. This naturally leads to using one or more gas Cherenkov counters.

3. Finally, while one does all that is possible to increase the acceptance of the device, it is clear from the cross-section estimates that very high luminosity is needed. This can be done by demanding the device be capable of operating at very high rates (greater than $10^{3}$ photons per second) with minimal deadtime, or by the brute-force technique of a long exposure (1000 hours). In practice both are necessary. 


\section{EQUIPMENT AND BEAM}

The experiment was conducted from winter 1977 to spring 1978 in the broad-band neutral beam located in the east area of the Proton Laboratory at Fermi National Accelerator Laboratory. Instead of presenting a comprehensive piece-by-piece description of the apparatus and beam line, this section will give a broad overview of both, mainly to establish the vocabulary. However, details of the experiment having an impact on the particular final states of interest here will be closely examined.

\section{A. The Beam}

The beam is formed by striking a $30-\mathrm{cm}$ beryllium target with the primary proton beam. Charged secondaries are removed with a series of sweeping magnets leaving a neutral beam composed of $\mathrm{K}_{\mathrm{L}}^{0}$ 's, neutrons, and photons. The photons are the result of $\pi^{0}$ decays. The beam is then passed through 105 feet of deuteriurn which, because of a high ratio of radiation length to interaction length, preferentially attenuates the hadronic portion of the beam. The neutrons are reduced by a factor of 200 with respect to their original fraction. The $\mathrm{K}_{\mathrm{L}}^{0}$ 's are reduced somewhat less because their hadronic cross section is $25 \%$ smaller than that of neutrons. The resulting photon beam has a $1 \%$ hadron contamination.

The photon spectrum is shown in Fig. 1 and above $50 \mathrm{GeV}$ can be characterized by $\exp \left(-\mathrm{E}_{\gamma} / 47\right)$. Running with $6 \times 10^{11}$ protons on target gives approximately $10^{7}$ photons above $50 \mathrm{GeV}$. The useful flux for a charmed particle search is in the energy range 50-180 


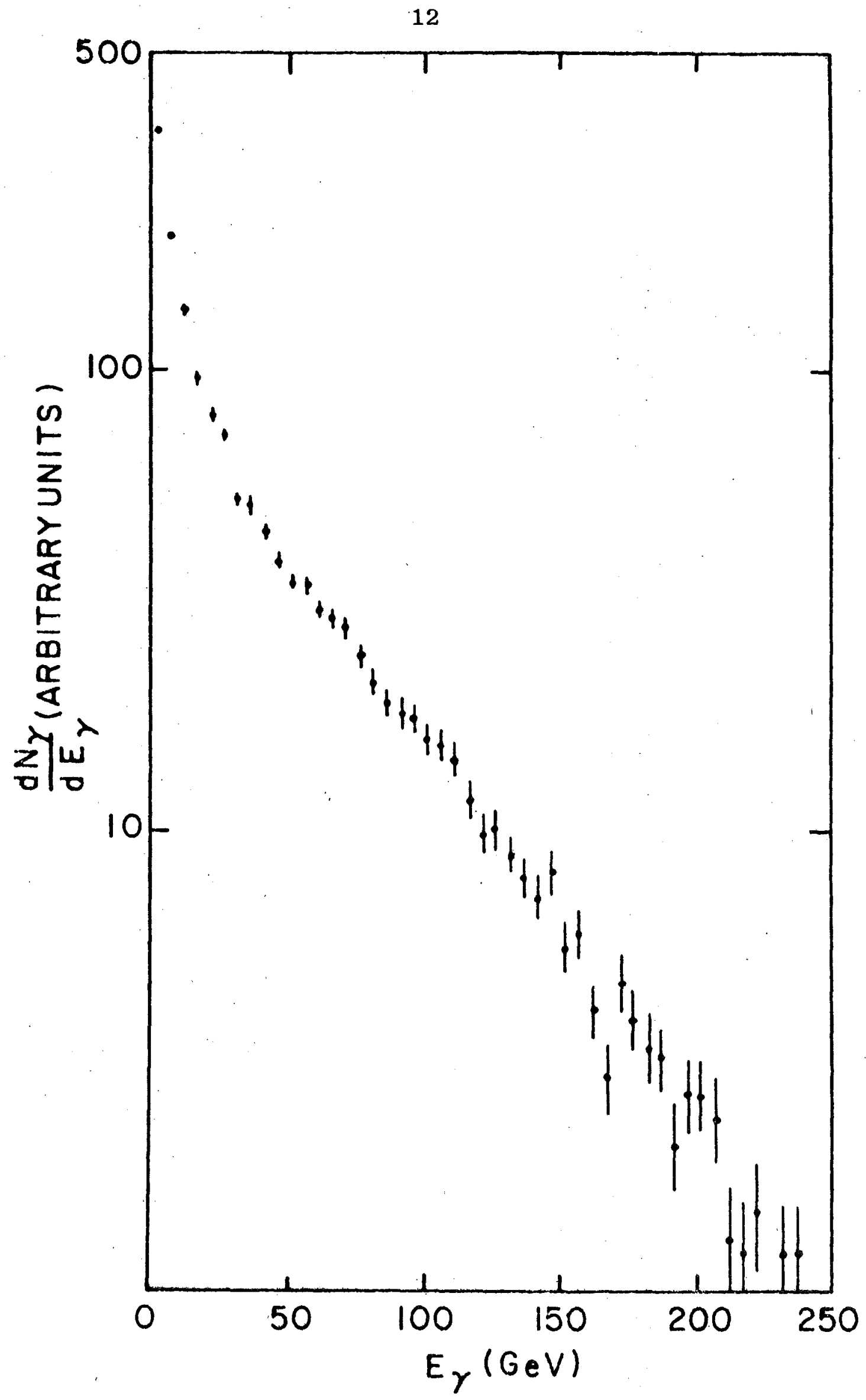

Fig. 1. Energy Spectrum of Photon Beam. 
GeV. The $K_{L}^{0}$ spectrum is less well understood but is probably similar in shape. The neutron spectrum is much stiffer with an average energy of about 0.8 of the primary beam energy (Fig. 2).

Although the number of neutral hadrons is two orders of magnitude less than the number of photons, their intrinsically larger cross section made the hadron and photon event rates roughly equal. To monitor the effect of the hadronic component approximately one-quarter of the data was taken with six radiation lengths of lead in the beam, effectively killing the photons. These runs are referred to as the $\mathrm{K}_{\mathrm{L}}^{0}$ runs although neutrons are also present. During these runs the raw trigger rates dropped a factor of two suggesting only half of the triggers were photon induced.

Beam quality and flux were monitored by two devices. The number of protons on target was measured by a Secondary Emission Monitor (SEM). The total photon power was measured with a Wilson-type quantameter $(Q)$. The ratio of $Q /$ SEM served as a monitor of beam performance since the number of protons on target is linearly related to the total photon power. If this ratio deviated from its normal value, a bevy of loss monitors and gauges could be consulted to isolate the problem. These problems fell into two classes: Q/SEM too small usually indicated poor targeting of the primary proton beam, and Q/SEM too large usually was caused by partial venting of the liquid deuterium filters . 


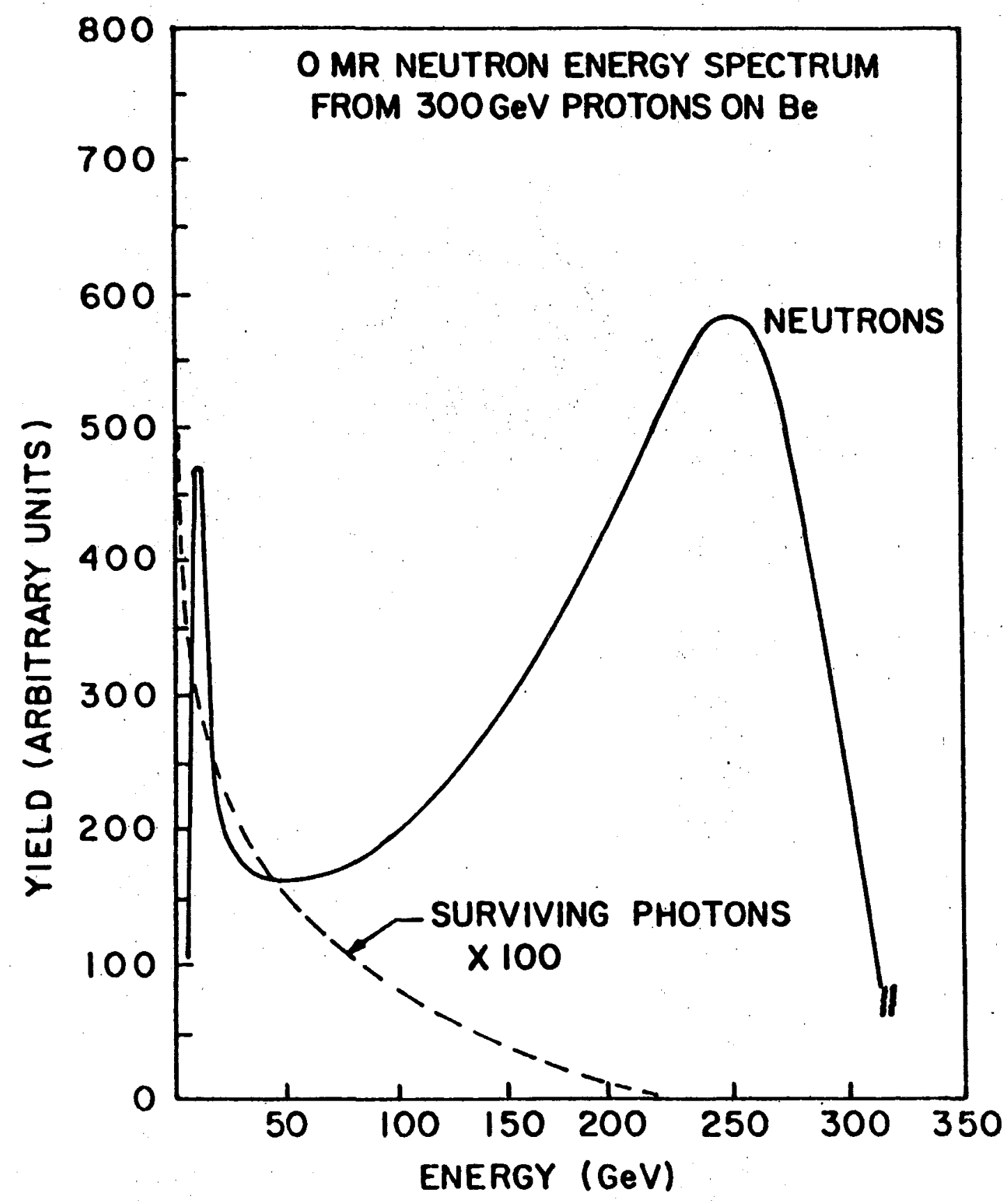

Fig. 2. Energy Spectrum of Neutrons. 
B. Charged Track Detection

Two types of measurements are critical to the analysis:

1. Charged-track detection and momentum analysis.

2. Charged particle identification.

The spectrometer (Fig. 3) consists of five proportional chambers. (P0-P4) and two analyzing magnets (M1 and M2). The chambers have three planes of wires; $x$, measuring in the non-bend plane, and $v$ and $u$ in narrow-angle stereo $(\tan \theta=1 / 5)$, measuring the bend direction. The narrow wire spacing, high segmentation (7000 wires), and arrangement of three chambers before the second analyzing magnet and two after, allows reliable reconstruction of events with ten or more tracks with relative ease. The desire to detect such high multiplicities with minimal confusion demands the chambers operate at a very high efficiency. In practice, efficiencies of greater than $98 \%$ were maintained on all planes. The chamber geometry is summarized in Table $I$.

The chambers were also instrumented with Time Recorder Modules (TRM). The chambers are divided into 32 regions, with the region widths varying depending on particle population. Timing is done on the or'd output of the amplifiers in each region by a cable clock running at 2.5 nanoseconds/count. Although up to thirty-two counts are available, the clock frequency and maxirnum drift time limit the needed range to about 10 divisions. Time slewing and other non-linear effects limit the obtainable resolution to about 3-4 divisions. The TRM output was also available for use in the trigger as will be described later. 

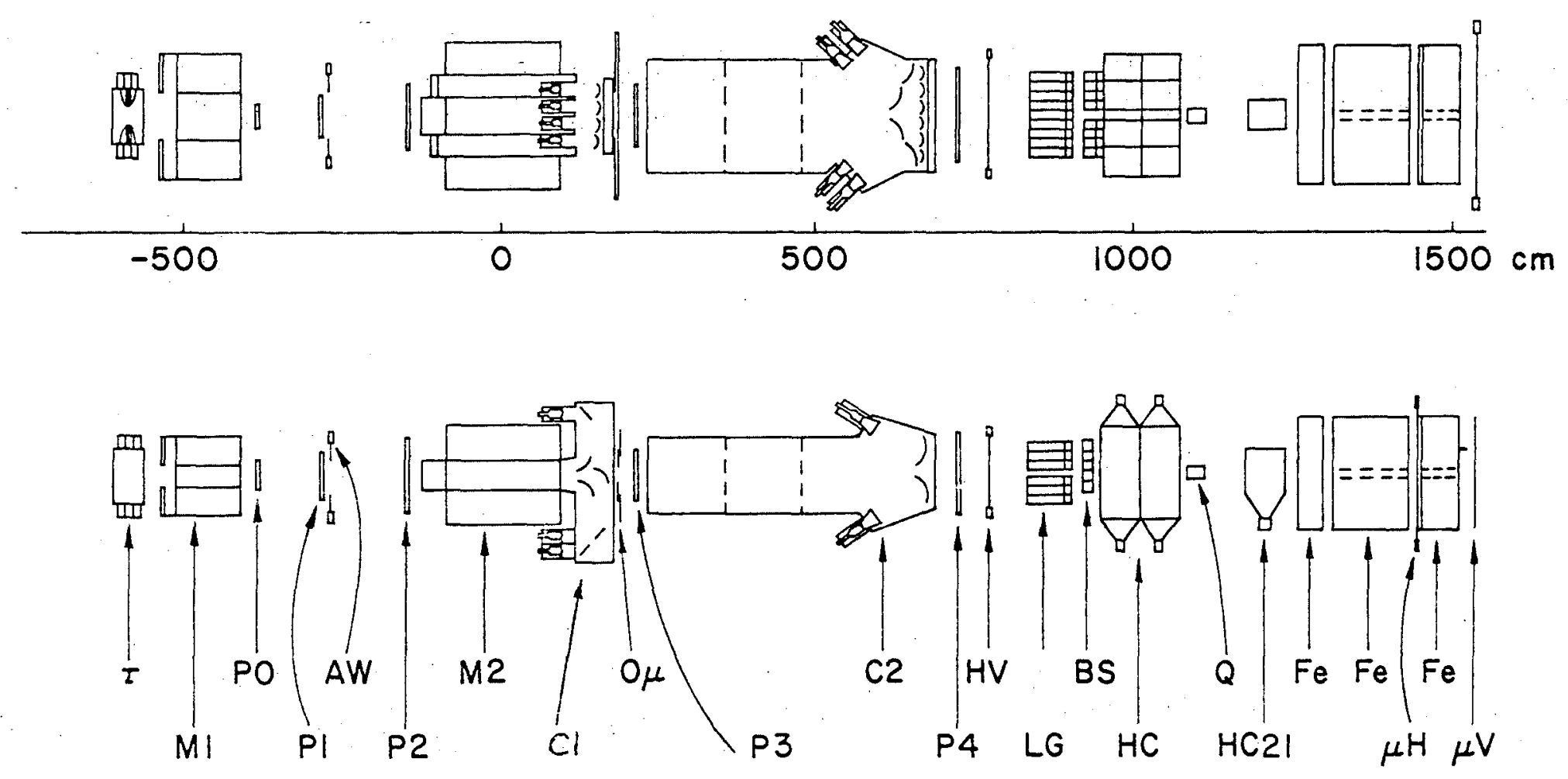

Fig. 3. Layout of Experimental Apparatus. 
TABLE I

CHAMBER PLANE NUMBER OF SIZE IN WIRE SPACING H.V.

\begin{tabular}{|c|c|c|c|c|}
\hline$=$ & WIRES & INCHES & IN $\mathrm{mm}$ & KV \\
\hline $\begin{array}{l}x \\
u \\
v\end{array}$ & $\begin{array}{l}288 \\
384 \\
384\end{array}$ & $11 \times 15$ & $\begin{array}{l}1 \\
1 \\
1\end{array}$ & 6.0 \\
\hline $\begin{array}{l}x \\
u \\
v\end{array}$ & $\begin{array}{l}256 \\
384 \\
384\end{array}$ & $20 \times 28$ & $\begin{array}{l}2 \\
2 \\
2\end{array}$ & 4.0 \\
\hline $\begin{array}{l}X \\
U \\
V\end{array}$ & $\begin{array}{l}384 \\
576 \\
576\end{array}$ & $32 \times 40$ & $\begin{array}{l}2 \\
2 \\
2\end{array}$ & 4.0 \\
\hline $\begin{array}{l}x \\
u \\
v\end{array}$ & $\begin{array}{l}416 \\
576 \\
576\end{array}$ & $32 \times 40$ & $\begin{array}{l}2 \\
2 \\
2\end{array}$ & 4.0 \\
\hline $\begin{array}{l}x \\
u \\
v\end{array}$ & $\begin{array}{l}336 \\
768 \\
768\end{array}$ & $40 \times 60$ & $\begin{array}{l}3 \\
2 \\
2\end{array}$ & 3.8 \\
\hline
\end{tabular}

PROPORTIONAL CHAMBERS INFORMATION 
The two magnets allow for the momentum measurement of two differing track types. Tracks which pass through to at least P3 are called inner tracks and those that do not are called outer. Much more will be said about the details in Chapter IV, but basically M1 measures the outer tracks and M2 measures the inner. The magnets were run with opposite relative polarities and effective momentum kicks of $0.40 \mathrm{GeV} / \mathrm{c}$ and $0.65 \mathrm{GeV} / \mathrm{c}$ for M1 and $M 2$, respectively. In addition to allowing the momentum measurement of outer tracks, M1 also sweeps and spreads the copiously produced point source of $\mathrm{e}^{+} \mathrm{e}^{-}$pairs into a two-inch wide vertical swath making life somewhat easier for the chambers. Even with M1, the chambers had to withstand a megacycle of $\mathrm{e}^{+} \mathrm{e}^{-}$pairs on the central wires. The currents are chosen so the pairs refocus in front of $\mathrm{P} 4$.

\section{Cherenkov Counters}

With charm decay modes emphasizing strange and heavy particles, a good particle identification system is necessary. Two segmented Cherenkov counters (C1, C2) attempt to fill this prescription. $\quad \mathrm{C} 1$ is a 120 -inch long nitrogen-filled radiator with 12-cell segmentation residing in M2's aperture. C2 is 180 inches of $80 \%$ helium and $20 \%$ nitrogen located between $\mathrm{P} 3$ and P4. The 16. phototubes of $\mathrm{C} 2$ had a jet of nitrogen flushed across their surface to prevent helium from diffusing through the windows and contaminating the tubes. The $\pi / \mathrm{K} / \mathrm{p}$ thresholds are $6 / 20 / 40 \mathrm{GeV} / \mathrm{c}$ in $\mathrm{C} 1$ and $12 / 40 / 75 \mathrm{GeV} / \mathrm{c}$ in $\mathrm{C} 2$. The Cherenkov counter's vital statistics are given in Table II and geometry in Figs. 4 and 5 . 


\section{CHERENKOV COUNTER SUMMARY}

\begin{tabular}{|l|c|c|c|c|}
\multicolumn{2}{c}{ LENGTH } & GAS & \multicolumn{2}{c}{ CELLS $\pi / \mathrm{K} / \mathrm{p}$} \\
\hline C 1-RADIATOR & $120^{\prime \prime}$ & $100 \% \mathrm{~N}_{2}$ & 12 & $6 / 20 / 40 \mathrm{GeV} / \mathrm{c}$ \\
\hline C2 RADIATOR & $180^{\prime \prime}$ & $20 \% \mathrm{i}_{2}+80 \% \mathrm{He}$ & 16 & $12 / 40 / 75 \mathrm{GeV} / \mathrm{c}$ \\
\hline
\end{tabular}

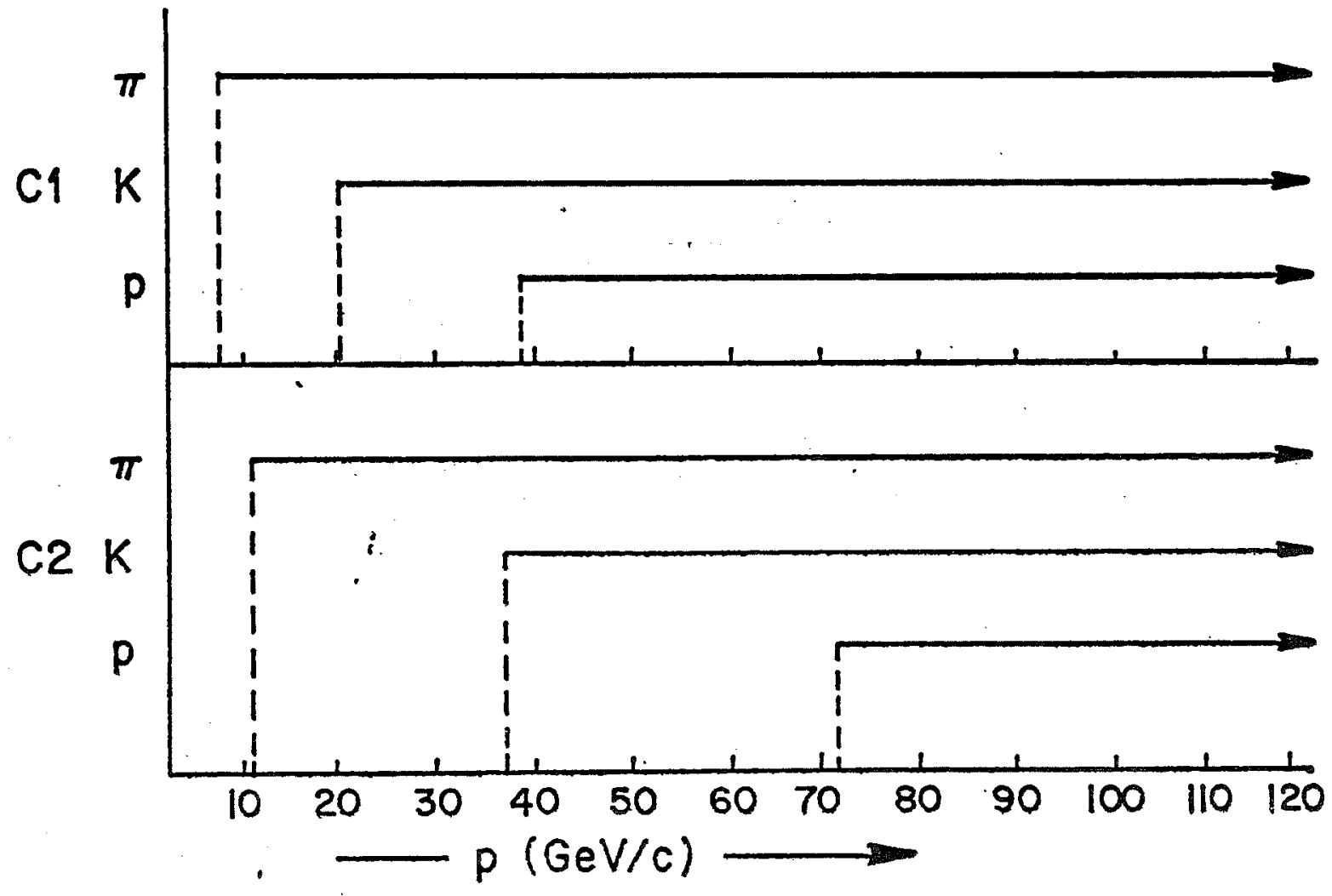



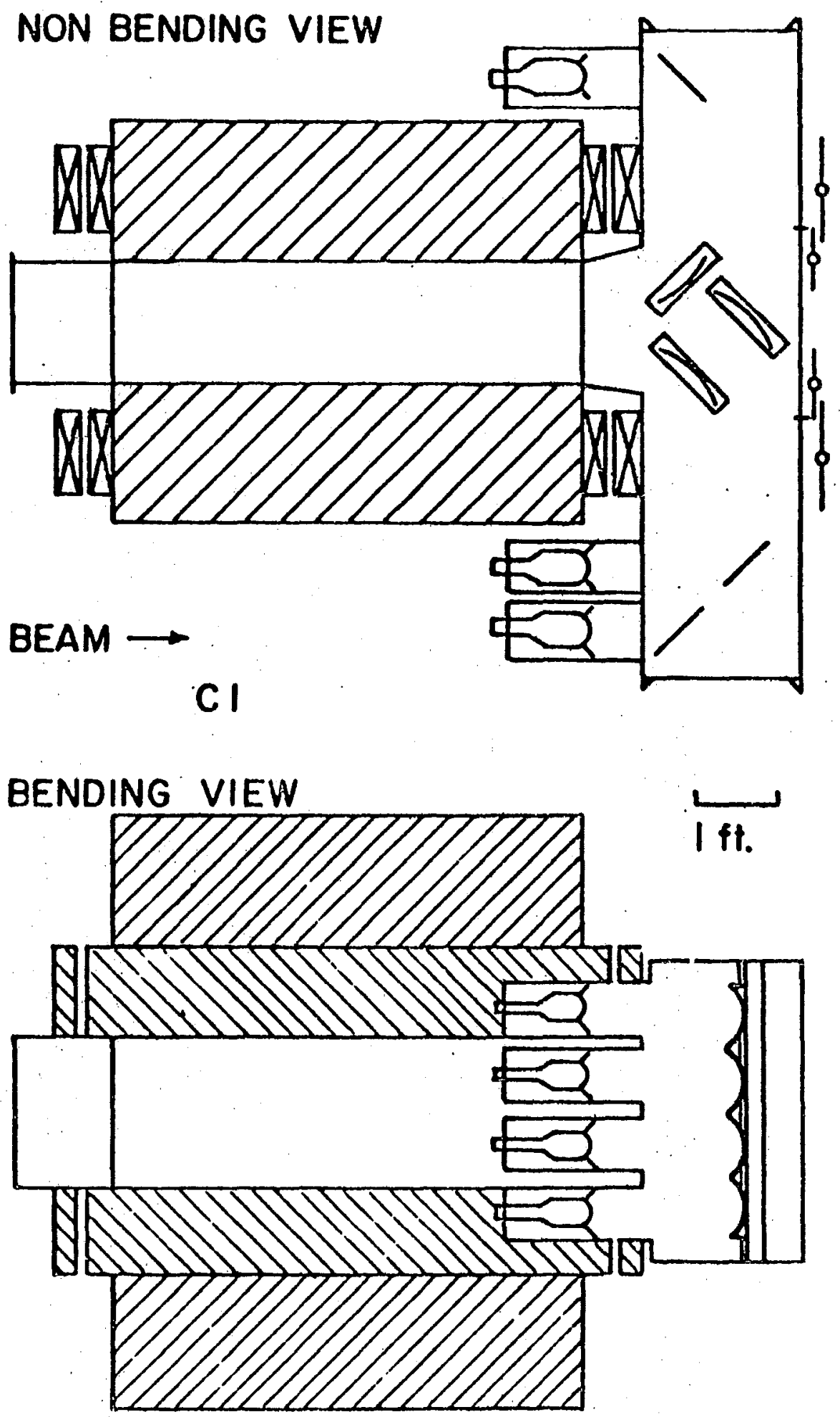

Fig. 4. Geometry of C1. 


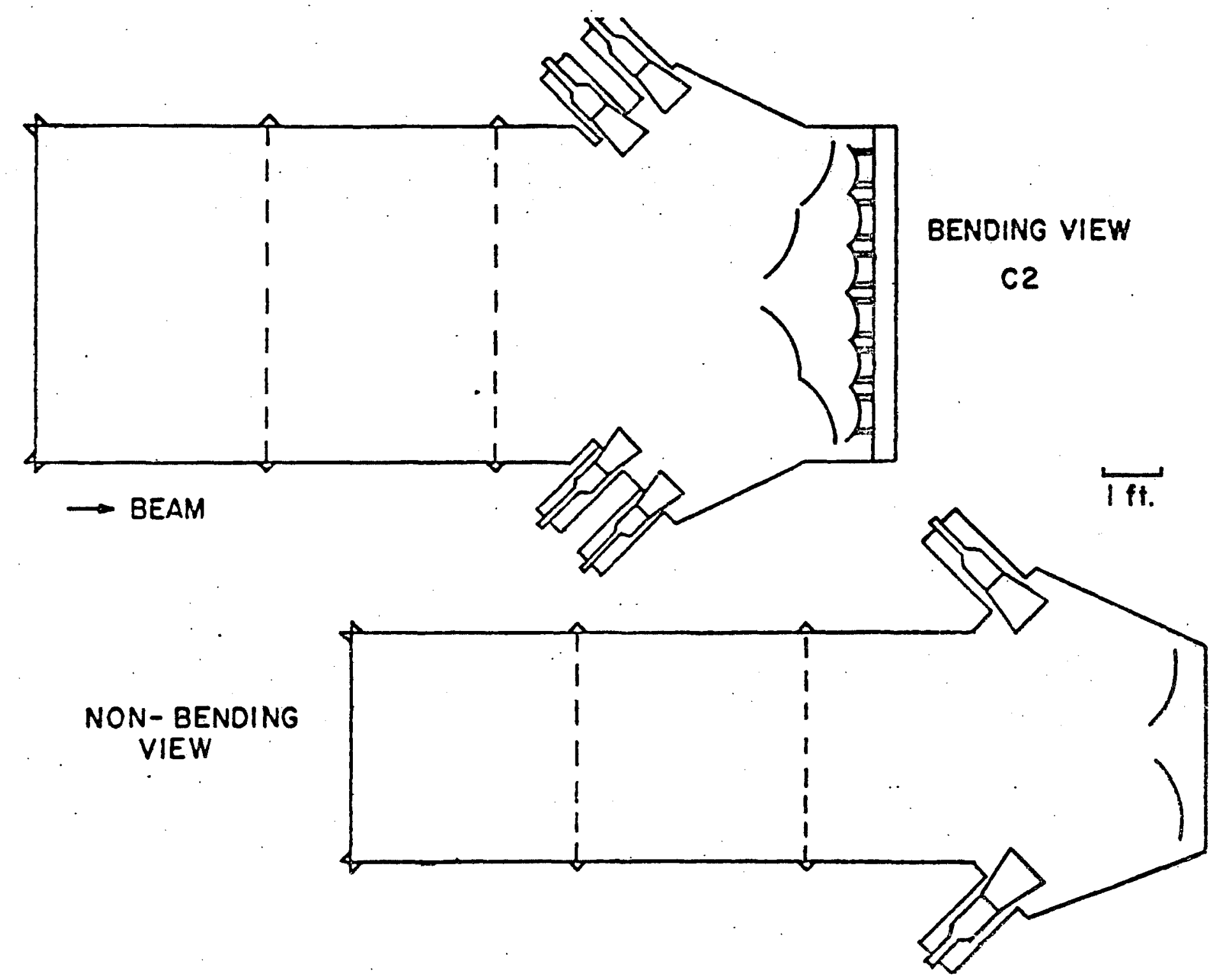

Fig. 5. Geometry of C2. 
D. Other Features

Given these two necessities of charged track detection and identification one can now add other features. The target was a collection of 20 individually wrapped $1 \mathrm{~mm}$ by 2 -inch square scintillators each viewed by an RCA 4622 phototube (Fig. 6). The target represents $4 \%$ of a radiation length, small enough so that the multiple scattering and reinteraction probability is low even for high multiplicity events. The target phototubes needed to be heavily shielded from M1's fringe field.

Two banks of scintillation counters, one horizontal (H) and one vertical (V), hang behind $\mathrm{P} 4$ (Fig. 7). These counters along with a coincidence in the last two target counters provide the lowest level trigger for the experiment, the master gate. There are $20 \mathrm{H}, \mathrm{V}$ counters in $\mathrm{all}, 8$ in the vertical plane and 12 in the horizontal plane. Each plane is split by a 4-inch vertical gap in the center allowing the $\mathrm{e}^{+} \mathrm{e}^{-}$pairs to pass through undetected.

A large array of 90 lead glass blocks is placed behind the H and $V$ counters providing $\pi^{0}$ and photon detection (Fig. 8). The blocks were calibrated on a run by run basis with a light flasher system, and on a long term basis by sweeping the pairs into the blocks during special runs. Forty-two small 2.5-inch square blocks are surrounded by 48 larger 6.0-inch square blocks. The stack was also split to allow the pairs to pass unscathed. Allowing the pairs to strike the lead glass would have resulted in yellowing of the blocks. The phototubes on the small blocks were RCA 6342A's. While these tubes had typical rise times of 15 nsec, the RCA 8055's on the large blocks were slow enough such that event overlap was possible. 

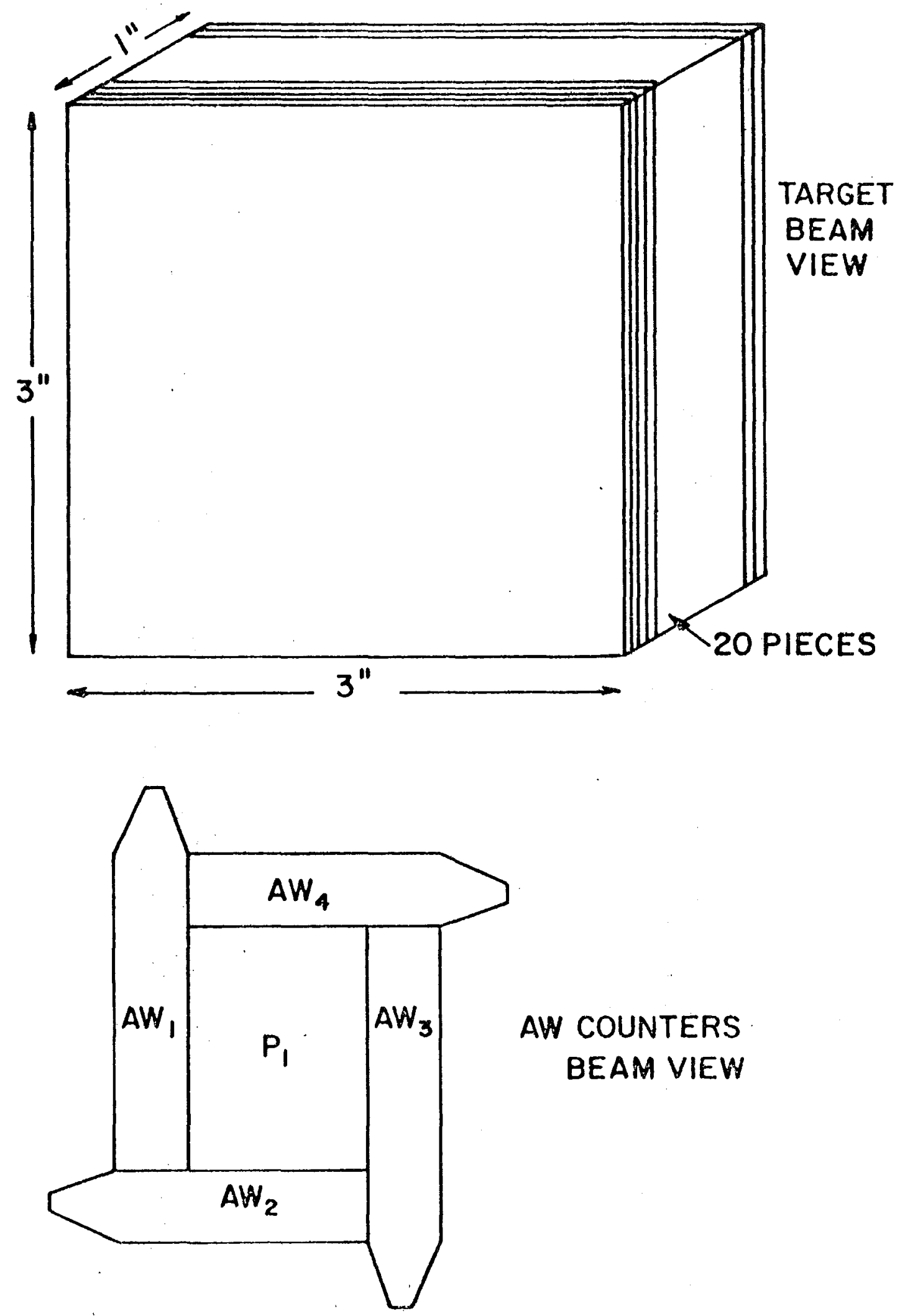

Fig. 6. Tau Target Counters (a) and AW Counters (b). 


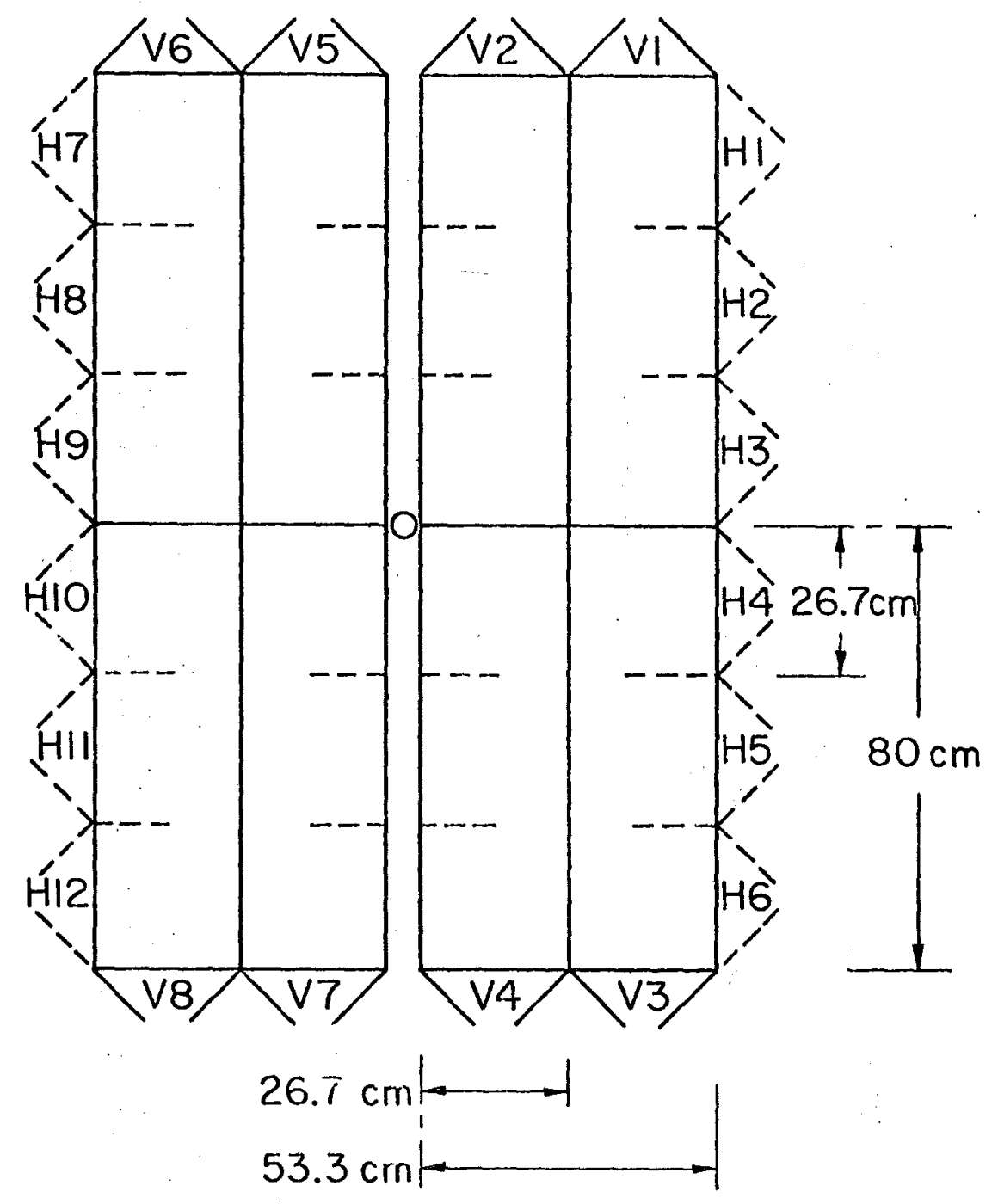

Fig. 7. Hx V Trigger Hodoscope Array. 


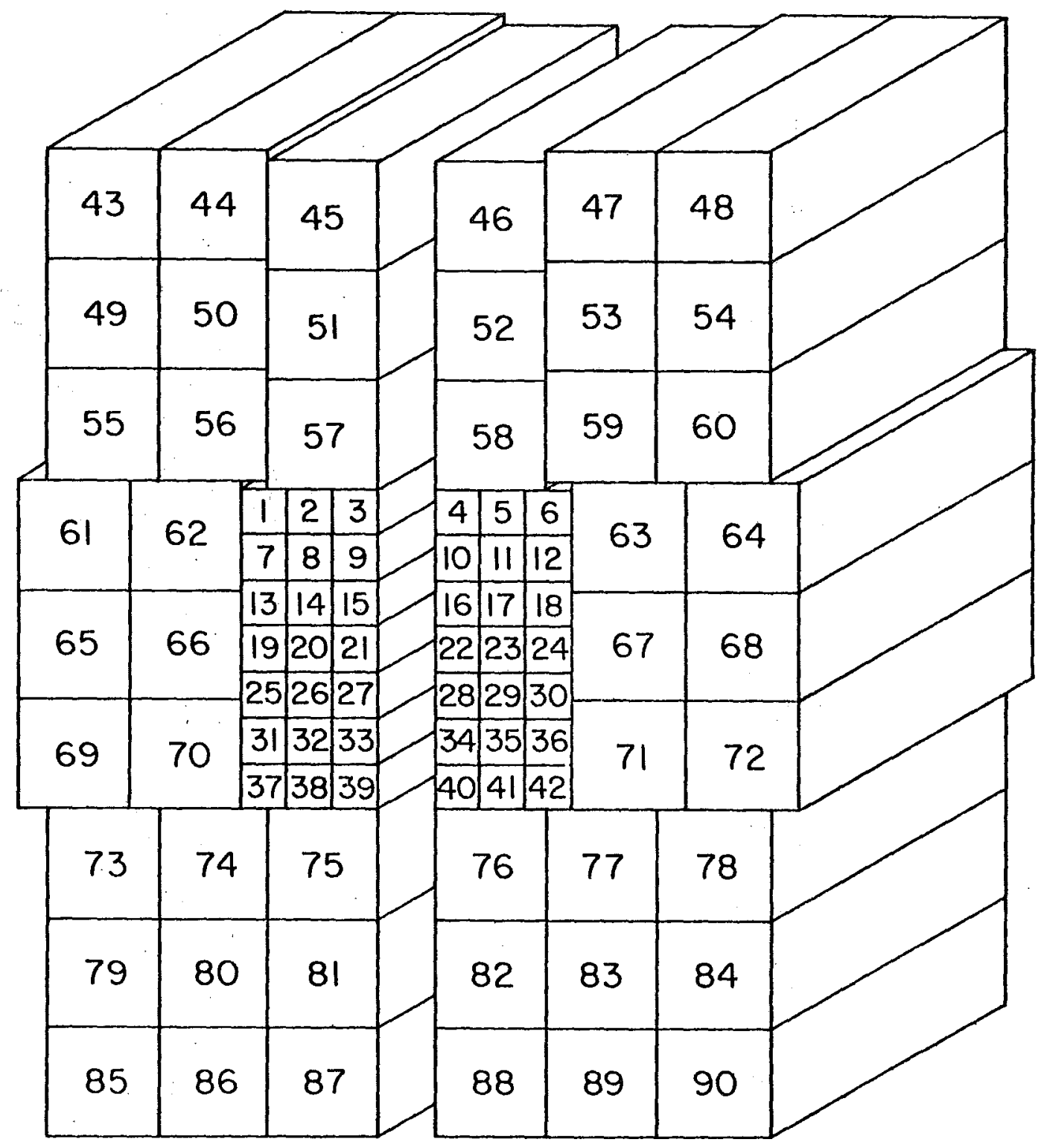

Fig. 8. Lead Glass Array. 
A low resolution, minimally segmented hadron calorimeter (Fig. 9) is next in line behind the lead glass stack and, as will be described later, is used in the trigger formation. The poor resolution of the calorimeter limits its use in the offline analysis to the same purpose as online, an estimate of the total hadronic energy.

A miscellaneous collection of scintillation counters complete the apparatus. Muon identification is provided in two places. The $\mathrm{O} \mu$ 's are eight counters arranged on the downstream side M2 making use of M2's iron as a shield. Two arrays $(\mu \mathrm{H}, \mu \mathrm{V}$, Fig. 10) are placed at the end of the apparatus between and behind large masses of stee1. There are 22 counters in the upstream vertical array and 18 counters in the downstream horizontal array. Finally four counters (AW1-4) surround P1 just outside its active area to warn of wide-angle tracks which have escaped detection.

\section{E. Data Acquisition and Triggering}

As noted in Chapter I, very high interaction rates are needed to obtain the necessary sensitivity. The trigger, therefore, needs to be fast in order to cope with these rates, yet, at the same time, maintain the ability to efficiently select the topologies of interest. The most natural way of satisfying these somewhat conflicting requirements is through a two-tiered logic system. The first level, called the master gate, is formed by a module fondly dubbed the "confusion logic." By rejecting the $e^{+} e^{-}$pairs the confusion logic reduces the raw event rate to a level that the more sophisticated, and, therefore, necessarily 


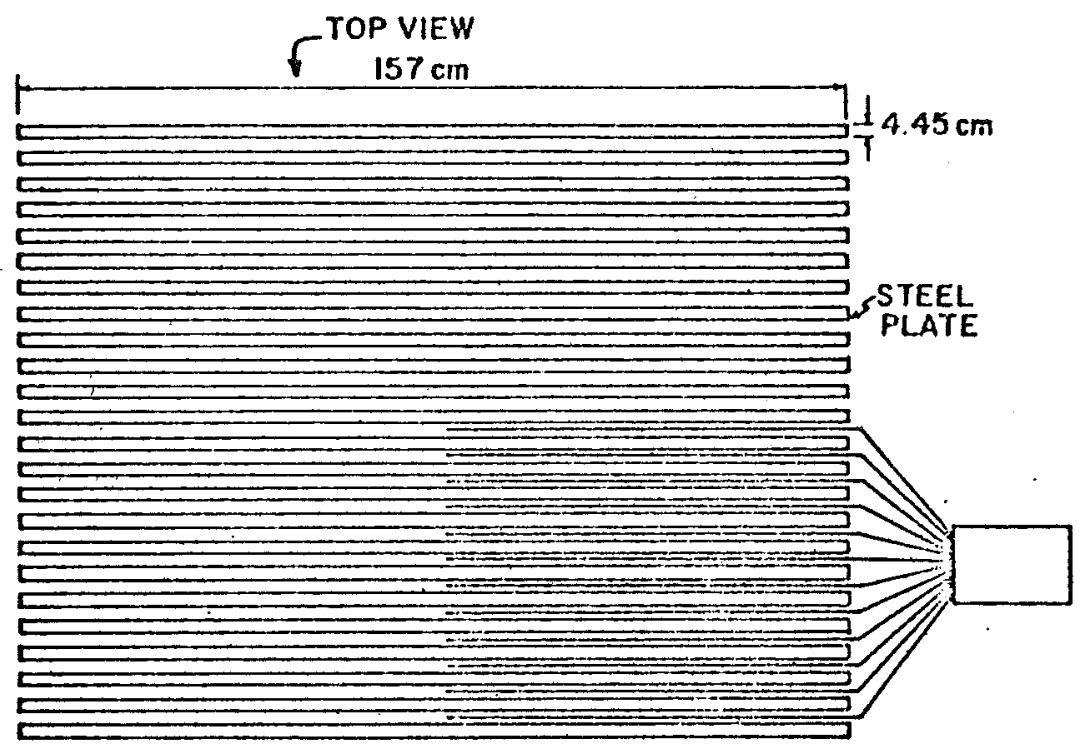

BEAM VIEW OF HADRON CALORIMETER

WITH DETECTOR ARRANGEMENT SHOWN

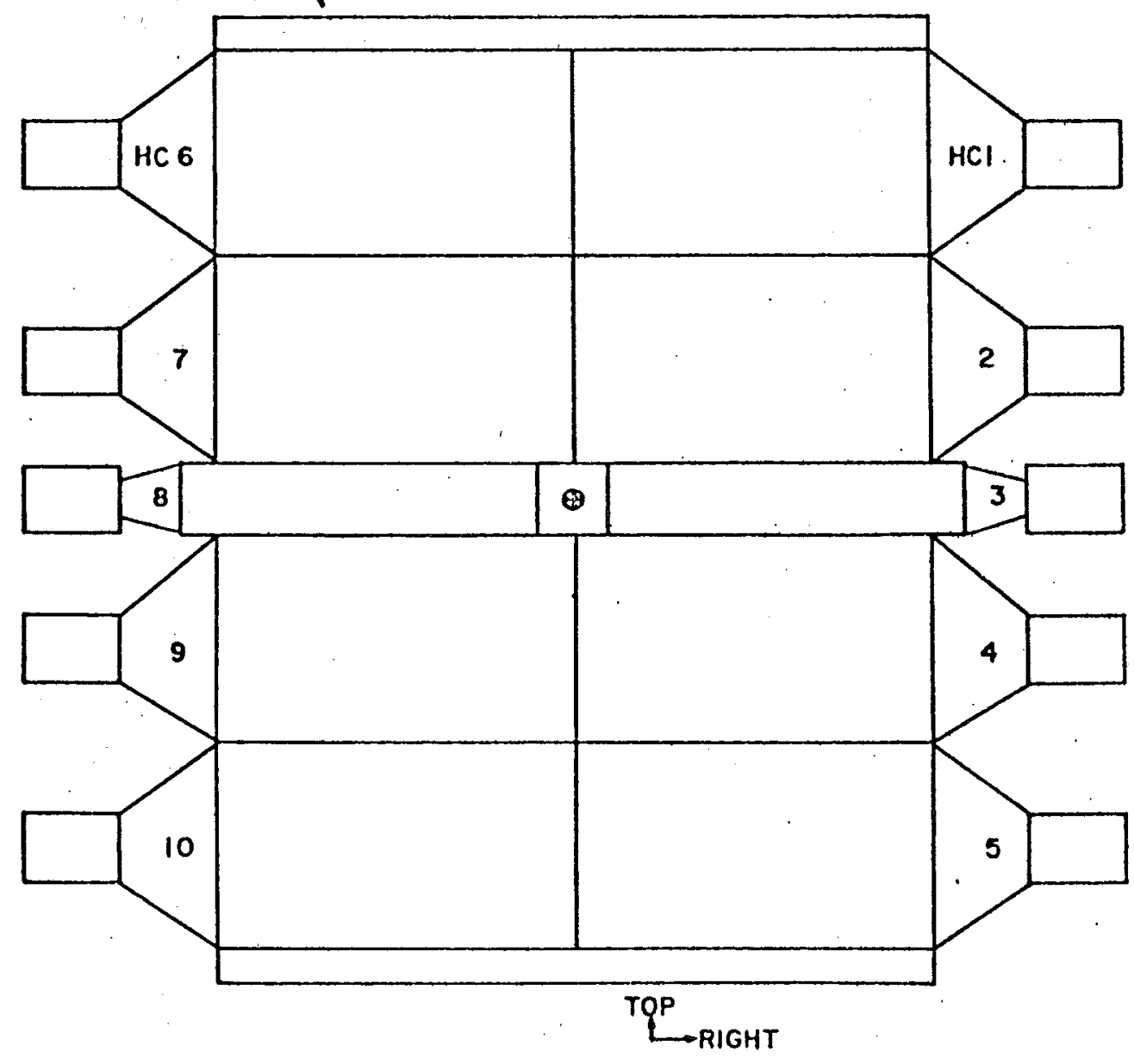

Fig. 9. Hadron Calorimeter. 


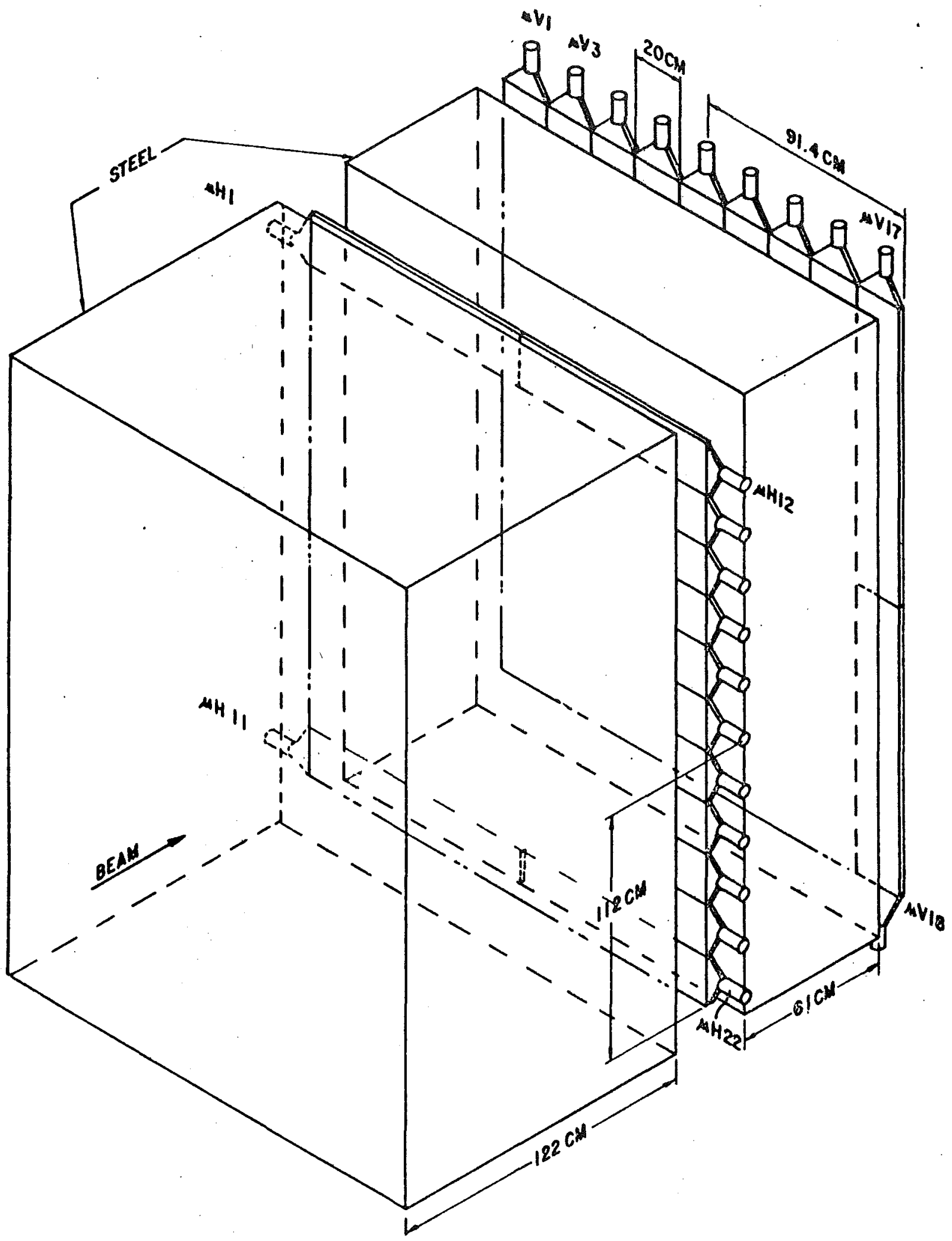

Fig. 10. Inner Muon Hodoscope Array. 
slower, second level of logic can cope with. This rejection is easily accomplished by putting a four-inch vertically centered gap in the $\mathrm{H}$ and $\mathrm{V}$ counter array. Thus, the master gate, which is formed by the coincidence of the or'd output of the $\mathrm{H}$ and $\mathrm{V}$ planes and the last two target counters, is not triggered by the pairs which are confined to the slot region. The confusion logic's second function is to generate signals, commonly called deadtime, which indicate when the system is unable to accept data. There are two primary sources of deadtime. The arrival of any signal to the confusion logic's inputs causes the generation of $100 \mathrm{nsec}$ of deadtime. This is meant to cover the memory or recovery time of the various detectors. Once a master gate is generated, the confusion logic waits $200 \mathrm{nsec}$ before clearing all latched information and allowing the generation of another master gate. This covers the slow logic decision time and is extended if the event is accepted to allow for event read-out time.

The time sequence is the following:

1. Form the coincidence of the tau target and HV counters.

2. If either is on (but not both), continue counting inputs but do not allow the formation of a master gate for 100 nsec.

3. If both on

(i) Activate the slow logic rack.

(ii) Latch all wire and counter information

(iii) Hold system for $200 \mathrm{nsec}$

(iv) Count the number of master gates that are being ignored so a deadtime correction can be made.

(v) If no blocking pulse originates from the slow logic system within 200 nsec, clear all latched information. 
The slow logic's function is to select the best 200 to 300 charm-event candidates from the 5000 hadronic events per pulse. Two sources of information are available to the slow logic rack, counter information and chamber information, provided by the TRM's. Both are "dc" in nature; that is, they are latched with the master gate and held until cleared. Timing of all counters need not be precise, the only requirement being that all information be available at the time the coincidence registers are strobed.

The counter information in the coincidence registers and the chamber information in the TRM's can then be combined into higher level logic decisions called buslines. Up to 16 buslines can be formed and pin logic modules can be easily set to require, veto, or ignore combinations of these buslines. The pin logic result is then passed through a prescaler which allows the collection of either all or some specified fraction of these triggers. The results of all pin logic modules are fed to the output trigger generator which signals the data acquisition system to record the event. The output trigger generator also sends a blocking pulse back to the confusion logic to prevent the clearing of the wire information and a pulse to generate the gates for all Analog-toDigital Converters (ADC's).

Once an output trigger is generated, the front end of the system is freed. The coincident registers are actually double buffered with one buffer holding the information that is to be recorded. The other buffer is free and the system continues to count all slow logic decision results while the data-acquisition 
system performs its function. This scheme allows for easy monitoring of deadtime effects. Unfortunately, the system was slightly corrupted with the introduction of the TRM's. Since they do not have the double buffering scheme of the coincident registers, the deadtime cannot be monitored for TRM-derived buslines (and necessarily for the triggers involving these buslines). Consequently, it is necessary to make the assumption that the deadtime of these triggers is the same as those without TRM logic.

The collection of buslines used in this experiment is long. What will be done here is to describe in words, rather than logic diagrams, the buslines and triggers relevant to this work. A complete description is given by Figs. 11(a-c). The organization of these buslines into triggers is given in Table III.

The relevant trigger involves four buslines:

1. $x_{\text {in }}>1$. This demands that at least two TRM regions in the $x$ planes of P2-P4 outside of the $e^{+} e^{-}$band be on.

2. High multiplicity veto. The median number of hits from each chamber is required to be less than 11 .

3. Greater than two-body. This demanded evidence in the chambers for more than two tracks passing all the way through to $\mathrm{P} 4$.

4. Energy busline. This is actually two lines. One requires a deposition of more than $50 \mathrm{GeV}$ in hadron eneriby as measured by the hadron calorimeter. The other demands the total energy seen by the calorimeter and lead glass exceed $60 \mathrm{GeV}$.

The trigger is aimed at selecting events with a significant hadronic energy content and multiplicities typical of those expected from the production and decay of charmed particles. 

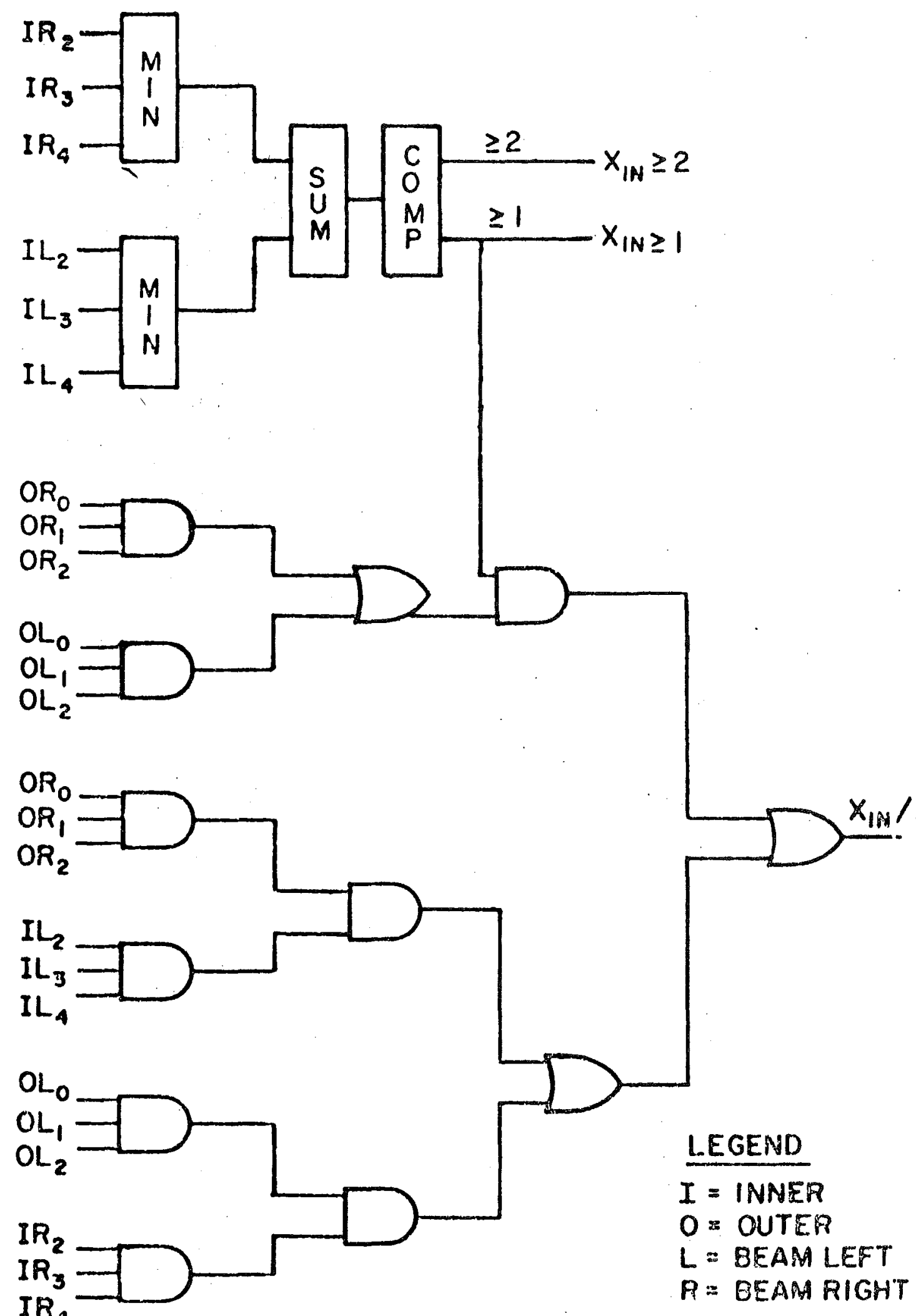

Fig. 11a. X-Logic Buslines. 

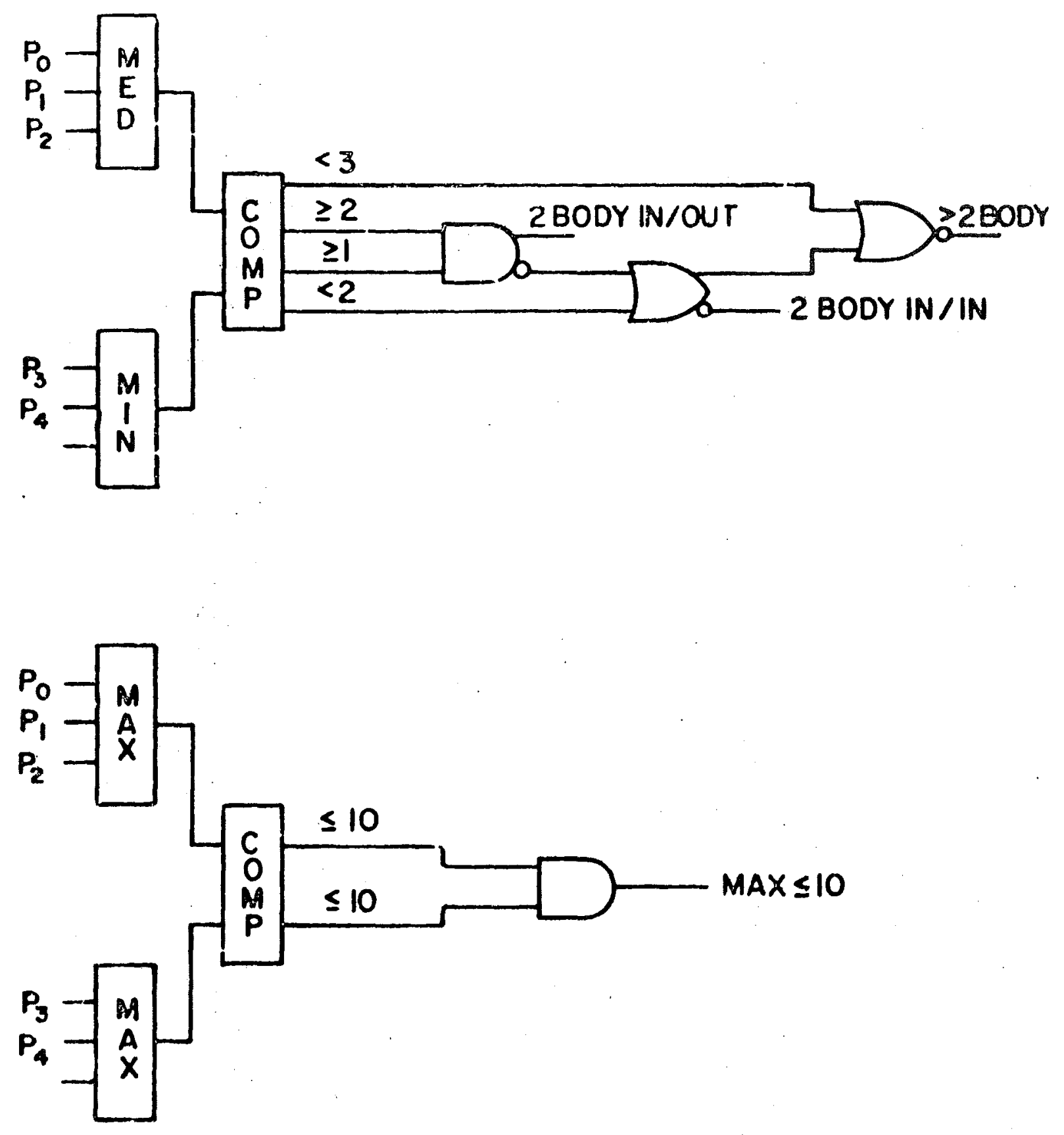

Fig. 11b. Multiplicity Buslines. 


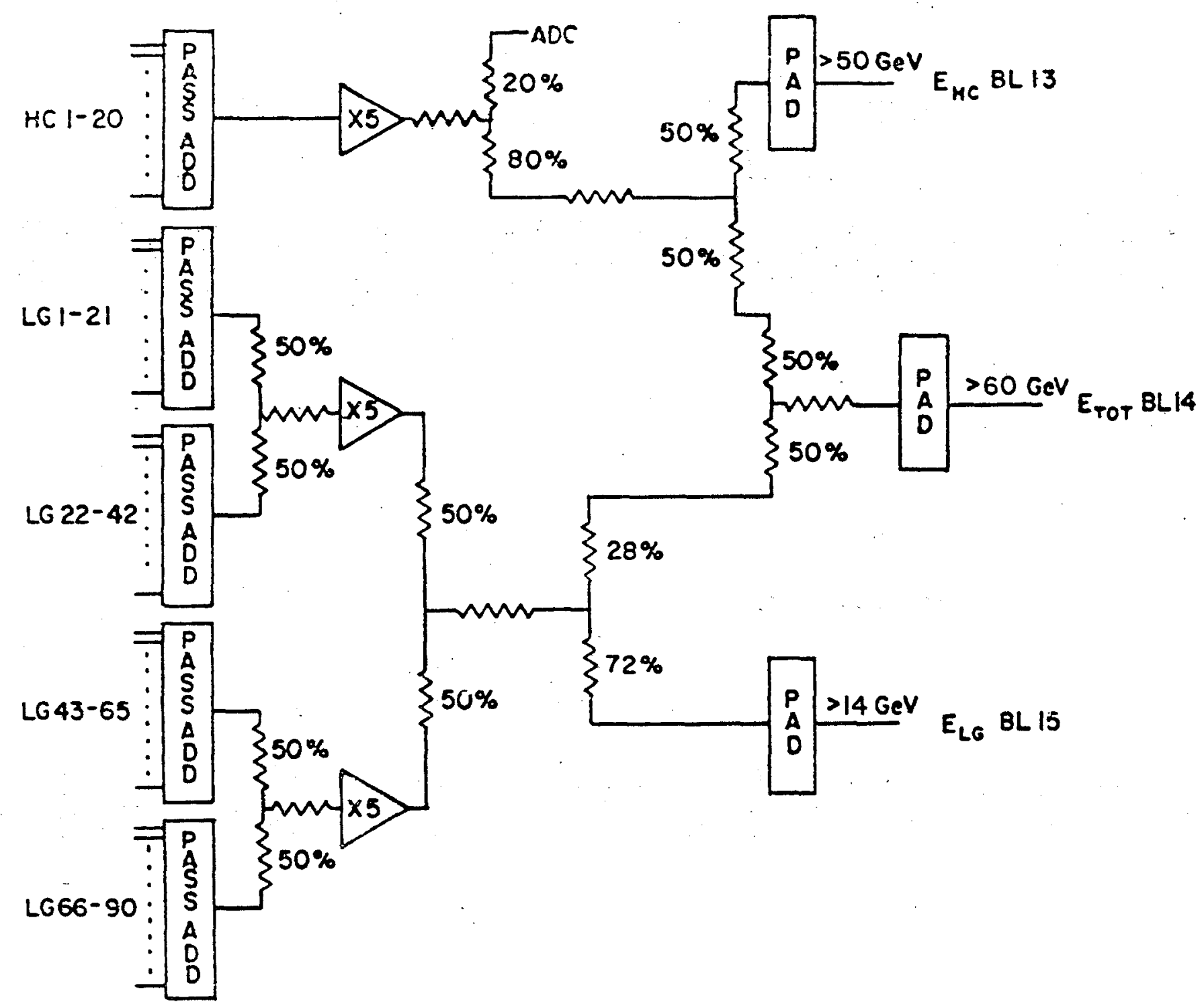

Fig. 11c. Energy Buslines. 
TABLE III

\begin{tabular}{|c|c|c|c|c|c|c|c|c|c|}
\hline $\begin{array}{l}\text { Bus Lin Logic } \\
\text {-ine }\end{array}$ & $\begin{array}{l}2 \mu \\
\mathrm{in} / \mathrm{in}\end{array}$ & $\begin{array}{l}\text { PIN } \\
2 \mu \\
\text { in/ol } \\
\end{array}$ & $\begin{array}{l}\text { OGIC } \\
2 \mathrm{e} \\
\mathrm{in} / \mathrm{in}\end{array}$ & & $\begin{array}{l}\text { USLINE } \\
\text { Hadrons }\end{array}$ & Mwpc & $E_{\text {TOT }}$ & $\begin{array}{l}\text { ise } \\
\text { in/in } 0\end{array}$ & $\begin{array}{c}\mu e \\
\text { out/in }\end{array}$ \\
\hline$x_{\text {in }} \geq 2$ & & & $\checkmark$ & & $\checkmark$ & $\sqrt{ }$ & & & \\
\hline$x_{\text {in }} / x_{\text {out }}$ & & $\checkmark$ & & & I. & & & & $\sqrt{ }$ \\
\hline$x_{\text {in }} \geq 1$ & $\sqrt{ }$ & & & & & & & $\sqrt{ }$ & \\
\hline $2 \mu_{\text {in }}$ & $\sqrt{ }$ & & & & & & & & \\
\hline$\mu_{\text {in }}$ & & $\sqrt{ }$ & & & & & & $\checkmark$ & \\
\hline$\mu_{\text {out }}$ & & $\sqrt{ }$ & & & & & & & 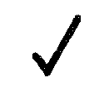 \\
\hline $\begin{array}{c}\text { AW } \\
\text { left + right }\end{array}$ & $x$ & $x$ & $x$ & & & & & $x$ & $x$ \\
\hline Max. $\leq 10$ & $\checkmark$ & $\checkmark$ & & & $\checkmark$ & $\checkmark$ & & $\checkmark$ & $\checkmark$ \\
\hline $\begin{array}{l}2 \text { body } \\
\text { in / in }\end{array}$ & $\sqrt{ }$ & & $\sqrt{ }$ & & & & & $\sqrt{ }$ & \\
\hline $\begin{array}{l}2 \text { body } \\
\text { in / out }\end{array}$ & & $\sqrt{ }$ & $\sqrt{ }$ & & & & & & $\checkmark$ \\
\hline$>2$ body & & & $x$ & & $\sqrt{ }$ & $\sqrt{ }$ & & $x$ & $x$ \\
\hline$E_{H C}$ & & & $x$ & & $\sqrt{ }$ & & $\checkmark$ & $x$ & $x$ \\
\hline$E_{\text {TOT }}$ & & & & & $\sqrt{ }$ & & $\sqrt{ }$ & & \\
\hline$E_{L G}$ & & & $\sqrt{ }$ & & & & & $\sqrt{ }$ & $\checkmark$ \\
\hline$A_{0}+A_{\mu}$ & $x$ & $x$ & $x$ & & $x$ & $x$ & $x$ & $x$ & $x$ \\
\hline
\end{tabular}


Once an event is accepted under any of the active triggers, a signal is sent to the Automatic Control Entry system (ACE). $\mathrm{ACE}$ is preprogrammed to read all the information which makes up an event. After ACE is triggered, it scans a prepared list of module addresses, transferring the information from the module to a 64,000 word memory. With a normal event length of 200-300 words, the memory could hold between 200-300 events per spill. The systen is designed to work on the same schedule as the accelerator. During the one second of beam, data are moved into the memory at one word per microsecond. At the end of spill ACE turns over control to the online computer which formats and writes the events off to tape before the next spill arrives about eight seconds later. Any spare computer time was devoted to inspecting a random sample of events. The emphasis was on hardware performance of both the equiptnent and data-acquisition system. No physics oriented monitor was attempted online. In addition, the computer handled the special monitoring tasks which included pedestal stability, lead glass gain tracking, and run summaries . 


\section{DATA ANALYSIS}

Charm spectroscópy demands the measurement of a large and diverse number of quantities. The characteristics and interplay of the devices measuring each of these quantities needs to be understood in detail before a charm search is attempted. This need is punctuated by the large data base with which one is confronted. Processing the 1600 raw tapes (representing 30 million events) taken during this experiment constitutes a considerable investment of both computer and physicist's time. An error in any of the many aspects of a charm search can result in a devastating loss of time. To meet this challenge a carefully designed sequence of programs was developed to handle those elements common to all analysis efforts. The sequence was broken into several sections guided by the philosophy of only doing those computations at the earliest stages of the analysis in which one had great confidence. This allows for an efficient use time, interleaving production running and program development time. These programs attempt to remain flexible in the face of seemingly mutually exclusive demands. (One state may demand a high efficiency in $\mathrm{K}_{\mathrm{S}}^{0}$ detection, while another wants only a very clean sample.) Once the data sample is reduced to more manageable proportions, each final state could be examined with programs individually tailored to its particular problems.

The data reduction process is divided into four phases:

1. Reconstruction

2. Higher Level General Analysis

3. Specific Final State Event Selection

4. Specific Final State Event Examination. 


\section{A. Reconstruction}

Aside from stripping ADC's not over their pedestal values and reformatting the data into a more usable form, this program is exclusively concerned with organizing the wire information into charge particle trajectories. Because of this, the program needs to know only the central wire positions and relative spacing of the 15 proportional chamber planes, both of which are easily found using data from special runs taken with the magnets off. Once these constants are determined, the reconstruction can be run in parallel with the data taking.

The trajectories which are found can be broken down by topology into three styles:

$$
\begin{aligned}
& \text { 1. Tracks } \\
& \text { 2. Stubs } \\
& \text { 3. VO's. }
\end{aligned}
$$

Tracks are formally defined as any charged particle trajectory which have proportional chamber strikes in chambers on both sides of M2. Tracks can be further subclassified into three categories characterized by the first and last chamber which have strikes: $\mathrm{P0}-\mathrm{P} 4, \mathrm{P} 0-\mathrm{P} 3, \mathrm{P} 1-\mathrm{P} 4$. The first, those having hits in al1 five chambers $\mathrm{PO}-\mathrm{P} 4$; are the most common and the best measured. P0-P3 tracks are possible because P3 covers a slightly larger aperture than P4. P1-P4 tracks are usually the result of a P0 inefficiency which, although small (1\%), appears to be correlated, i.e., when one plane fails, all fail. Stubs are charged trajectories which pass only as far as P2. After all tracks and stubs are found, the program attempts to collect any remaining hits into pairs of tracks consistent with a neutral $V$ decay 
originating between $\mathrm{P} 0$ and $\mathrm{P} 2$. Tracks emerging from neutral V's are required to pass through at least three consecutive chambers with the sole exception of a V0 composed of a track $\mathrm{P} 1-\mathrm{P} 4$ and a P1-P2 segment.

The pattern recognition always starts at the projection level $(x, v, u)$. The alternative method of organizing the $x, v, u$ triplets into points is less efficient. Projections are subsequently matched up using the $\mathrm{x}, \mathrm{v}, \mathrm{u}$ constraint. The number of shared or missing hits allowed depends on the style of track, becoming tighter as the redundancy is decreased.

After each track is found it is fit to an appropriate geometrical description. Tracks are fit to the five parameter description of two lines intersecting at the bend point of M2. The parameters are

1. $x_{0}:$ The $x$ coordinate of the track at the bend point of M2.

2. $\alpha_{M}:$ The $x$ slope of the track on the upstream side of

3. $y_{0}$ : The $y$ coordinate of the track at the bend point of M2.

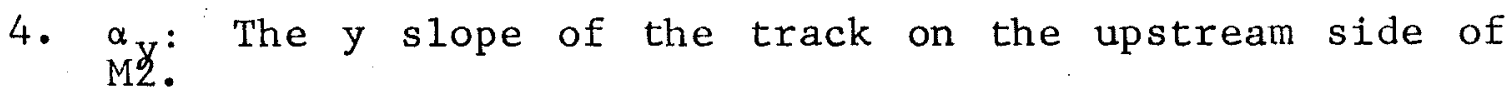

5. $\delta:$ The bend angle between the front and back $y$ line segments.

Stubs and Vo's are fit using a subset of these five parameters. The bend angle is unnecessary for stubs. VO's are fit subject to the constraint of a common vertex, thus removing one degree of freedom. Because the magnet has a finite length and fields in all three directions, corrections must be made to this simple description, allowing for a small bend in the $\mathrm{xz} p$ lane and a 
slight offset of the front and back halves of the track at the bend point. The parameterization is sufficient, and all these effects can be adequately handled using first-order corrections applied at the fitting stage. The fitting routine also scans the hits comprising the track removing, replacing, or adding hits from the available list whenever the resulting track is a better fit as measured by the $x^{2}$ per degree of freedom.

The pattern recognition and initial fitting proceeds independent of drift-time measurements. Only after all the charged particle trajectories have been found and fits performed is the drift-time information employed. Because the cell width is only $2-\mathrm{mm}$, the resolution of the left-right side ambiguity problem is somewhat more difficult and is handled differently than in a conventional drift-chamber system. The fit is performed iteratively, assigning drift distances only to those hits for which the track passes a sufficiently large distance from the struck wire. As more drift times are incorporated into the fit, the cutoff distance is allowed to slide closer to the wire reflecting the increase in resolution gained from the previous fit. The improvement in spatial resolution achieved by this technique was about a factor of two.

The output of the reconstruction program is written to 6250 BPI tapes allowing a condensation of the original $1600800 \mathrm{BPI}$ tapes to 200. This, coupled with the intrinsically greater reliability of the 6250 BPI tapes, eased the tape-handling problem considerably. 
B. Higher Level Analysis

The output of the reconstruction program is then passed to another major program. The program has a wide variety of duties with the ultimate goal of producing what can loosely be described as a four-vector tape. The list of tasks is as follows:

1. Assign momenta to all tracks.

2. Find a vertex if possible.

3. Assign momenta to all stubs assigned to the vertex.

4. Find VO's.

5. Perform the charged particle identification (Cherenkov analysis).

6. Match tracks to muon counters.

7. Find showers in lead glass.

The first four steps do not proceed linearly for reasons which will become apparent. For the final states considered here only the first five need to be examined in detail.

\section{Momenta and Vertex Determination}

The momentum of a track is a simple function of the bend angle and the amount of field experienced in passing through the magnet. The only complication is removing the $3-5 \%$ variation which occurs across the transverse directions of the magnet. Once the track's momentum is known, it can be traced through M1 to the center of the target.

All tracks and stubs contained within the target's transverse dimensions at its midplane are considered candidates eligible for the determination of a production vertex. After the distance of closest approach is computed, the list is then scanned for the track contributing the most to the $x^{2}$ of the fit. If any track is further away than 0.15 inch, it is 
rejected. The list of rejected tracks is scanned for possible additions and the process iterates itself until a stable solution is found. Multiple vertices are found by examining the list of leftover tracks. The two usual causes of a second vertex are an $\mathrm{e}^{+} \mathrm{e}^{-}$pair overlapping the event or the decay of a $\mathrm{V}$ close to the target volume. Figure 12 shows the $\mathrm{x}, \mathrm{y}, \mathrm{z}$ target distributions.

Stub momenta can be found once the vertex is established by picking the momentum which correctly swims the stub back to the $y$ of the vertex. Notice the stub momentum determination is subject not only to how much field the particle experiences, but also the distribution of the field. The intrinsic mathematical difficulties of inverting this problem were further compounded by the fact that M1 was far from an ideal magnet for two reasons. The magnet itself had no symmetry along the beam axis because of a field plate needed to shield the tau counter phototubes from the fringe field and an assymmetric coil configuration which was not centered in the iron. Early in the run one of M1's four coils shorted, resulting in an $x$ assymetry and non-negligible off-field components. Understanding of the $z$ distribution of the field is critical to recognizing $V$ decays in the magnet (Fig. 13). The $x$ assymetry and off-field components reduce the spatial and momentum resolution, ultimately causing poorer mass resolution, unless appropriate corrections for these effects are made.

This problem can be studied using the data. Figure 14 shows the distance a track misses the vertex in both the bend ( $y$ ) and non-bend $(x)$ views as a function of momentum. At high momentum $(>15 \mathrm{GeV} / \mathrm{c})$ the width of this distribution asymptotically 

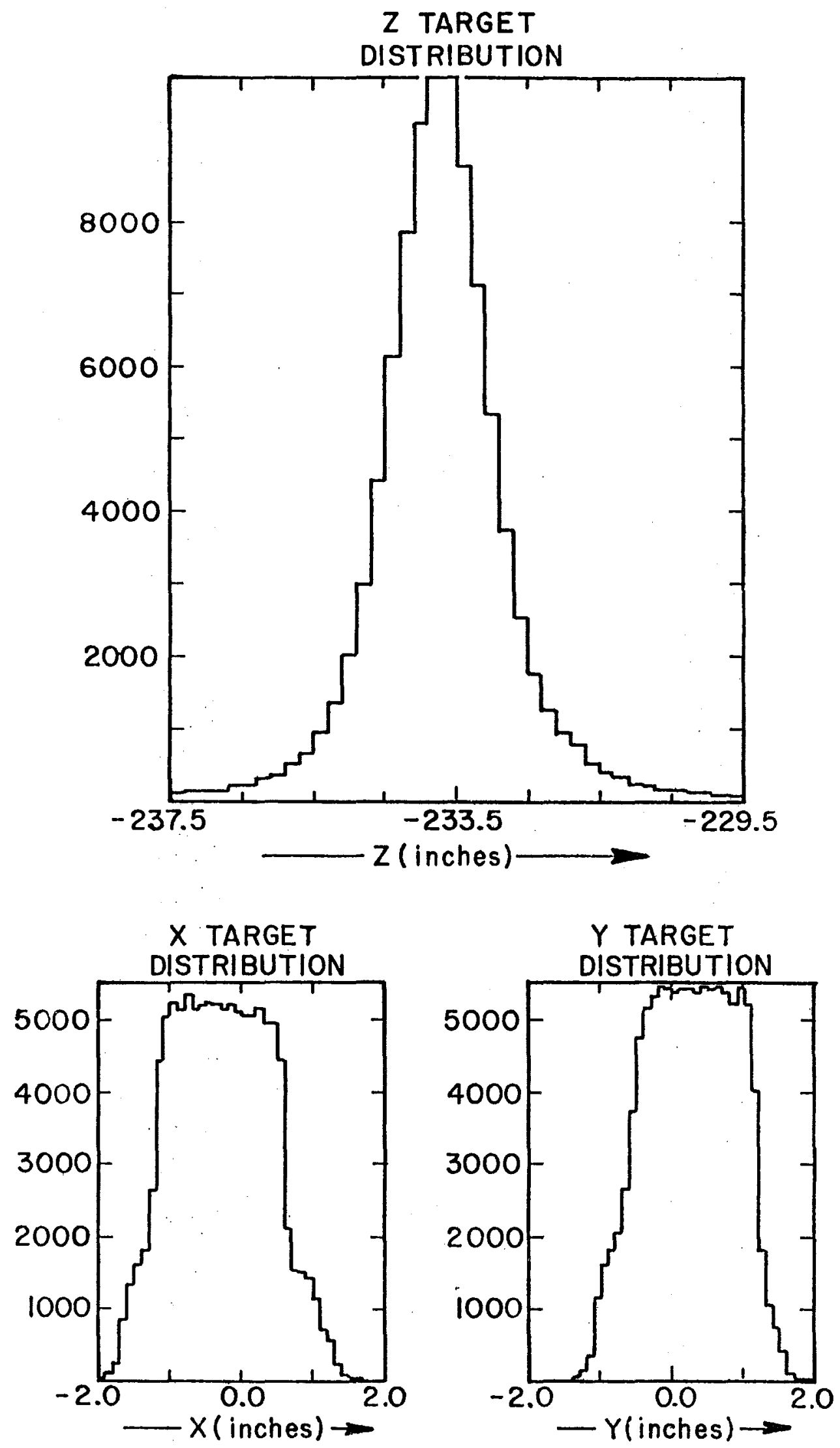

Fig. 12. Vertex Distributions. 


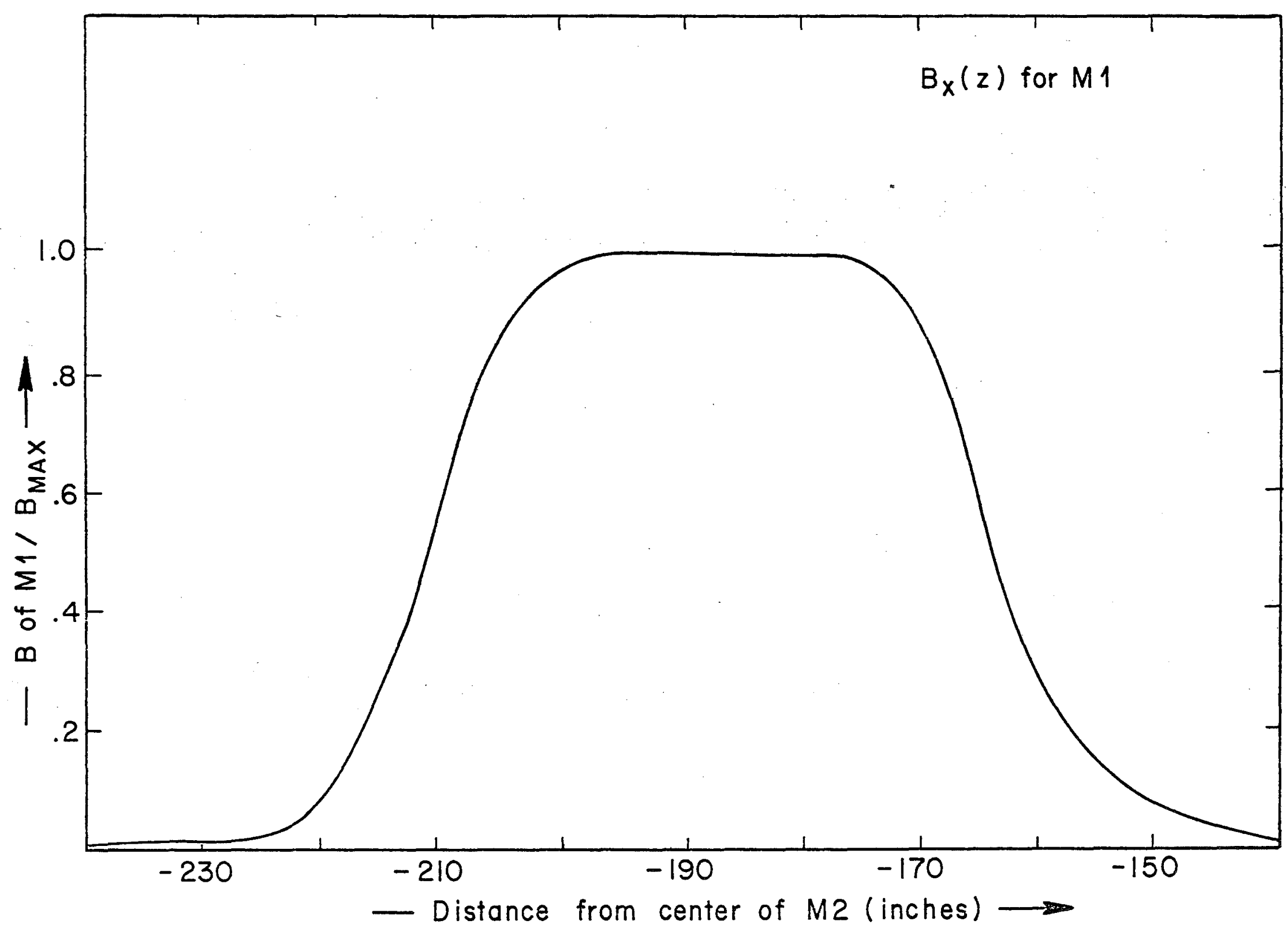

Fig. 13. Shape of Magnetic Field of M1. 

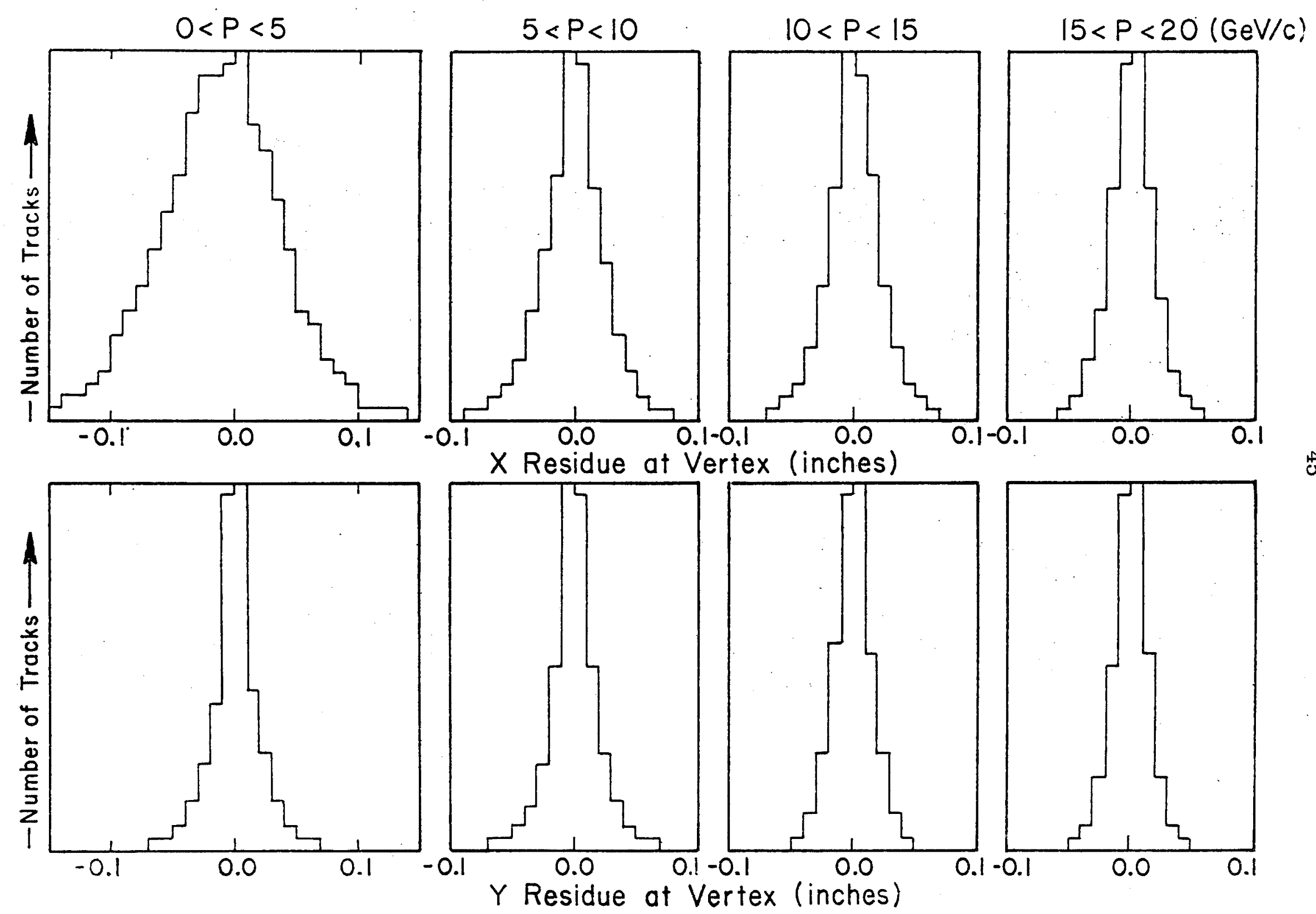

空

Fig. 14. Vertex Residue Distributions as a Function of Momentum. 

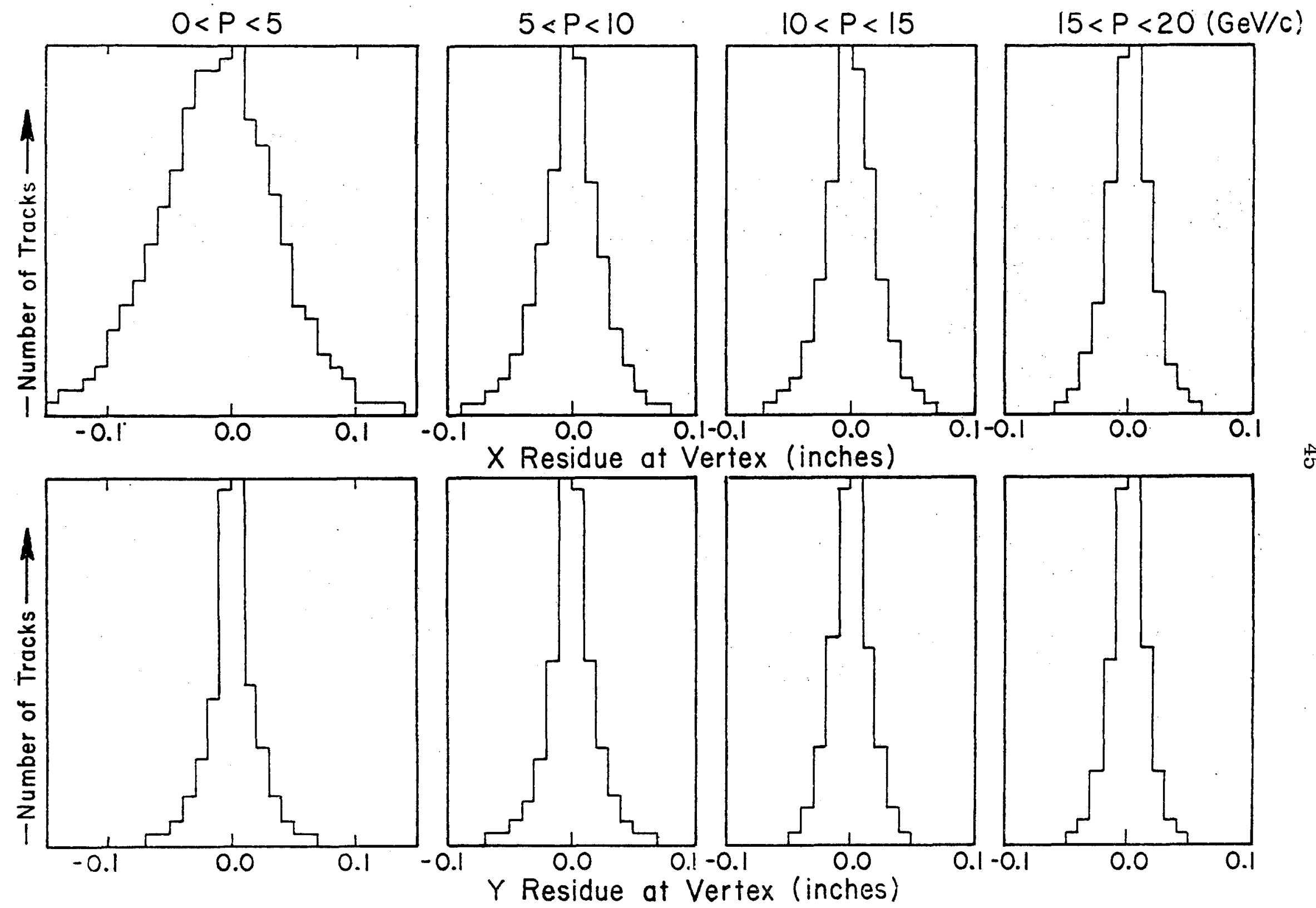

Fig. 14. Vertex Residue Distributions as a Function of Momentum. 
approaches the expected resolution of about 0.02 inch. At lower momentum, though, the width broadens in the non-bend view indicating there are fields of the order of $3-5 \mathrm{MeV} / \mathrm{c}$ effective kick which have not been compensated for. Multiple scattering cannot be the cause since the bend view shows no such broadening.

The momentum resolution of the inner and outer spectrometers can be roughly characterized by the functional form

$$
\begin{aligned}
\Delta \mathrm{p} / \mathrm{p}^{2} & =2.5 \% \text { at } 100 \mathrm{GeV} / \mathrm{c} \text { (inner) } \\
& =7.5 \% \text { at } 100 \mathrm{GeV} / \mathrm{c} \text { (outer) }
\end{aligned}
$$

Because the average momentum of particles in the outer detector is lower (Fig. 15), the effect of including stubs in mass calculations is not as bad as it might appear. The resolution for the inner tracks is determined by looking at the width of the $\psi$. The outer spectrometer resolution is found by comparing the momentum determined using $M 1$ with the result of the inner spectrometer calculation for tracks common to both. Unfortunately this number cannot be checked in the low-momentum region (below $7 \mathrm{GeV} / \mathrm{c}$ ), because these particles rarely pass through the inner detector. This is the momentum region where the lack of knowledge about M1's field can cause problems. Figure 16 shows the quantity $\left(\mathrm{P}_{\mathrm{STUB}}-\mathrm{P}_{\mathrm{TRACK}}\right) / \mathrm{P}_{\mathrm{TRACK}}$ (where $\mathrm{P}_{\mathrm{STUB}}$ means computing the momentum of a track as if it were a stub) as a function of track momentum. If all systematic effects have been removed, the width of this distribution should be constant as a function of momentum.

2. VO Identification

The Vo program attempts to find as many pairs of oppositely charged tracks which intersect a separable distance from the target. Cleanliness of the sample is not a major consideration. 
. 

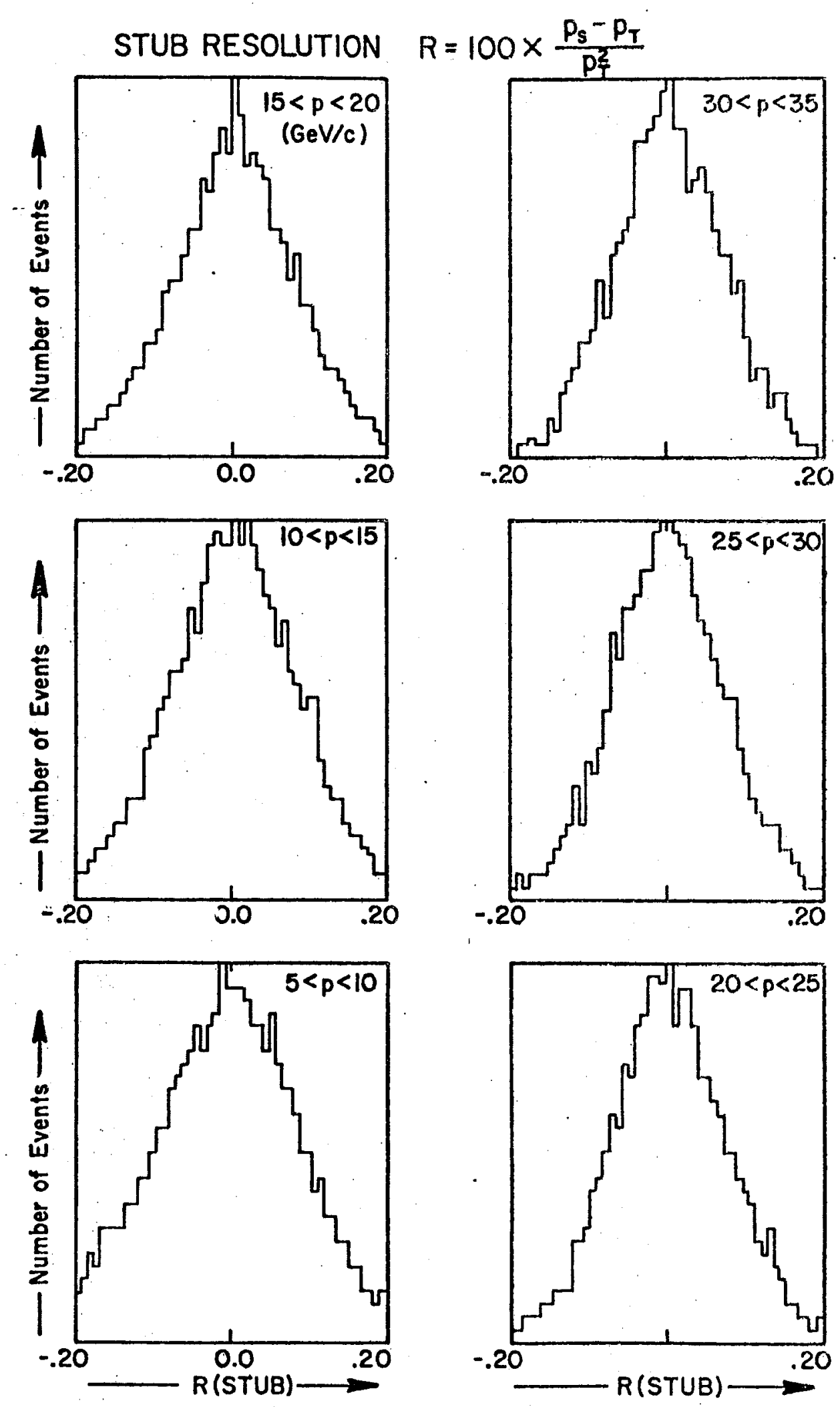
This tack is taken because the character of the V0 sample needed varies depending on the specific use. Although other types of information, most notably the Cherenkov counter pulse heights, are potentially useful at this point, it was found to be undesirable to attempt to use all information relevant at this point in the analysis.

The Vo's are organized into topologically distinct categories:

1. Downstream. These are the Vo's which decay in the volume between $\mathrm{PO}$ and $\mathrm{P} 2$ found by the reconstruction program. They come in three types.

(i) Clean downstream's pass the most stringent hit requirements with each track passing through at least three consecutive chambers (Case 1 Vo's).

(ii) Track-stub downstream VO's are formed by linking a P1-4 track with a pair of triplet hits in P1 and P2 which form a line consistent with an intersection between PO and P1 (Case $5 \mathrm{VO}^{\prime} \mathrm{s}$ ).

(iii) Dirty downstream's are of the same topology as the clean category, but the hit restriction and sharing requirements are considerably relaxed. These are the last thing looked for by the reconstruction program (Case $4 \mathrm{VO}^{\mathrm{S}} \mathrm{s}$ ).

The VO program's only duty here is to compute two masses for each VO candidate, one a $\pi \pi^{-}$mass and the other assigning the faster track the proton mass for a pi mass. In addition, the program checks the vertex assignment and flags the $V O$ as assigned or unassigned to the primary vertex. The mass calculation for track-stub downstream V0's by necessity assumes target assignment so that it can calculate the momentum of the stub by balancing the perpendicular mornentum about the line drawn from the event vertex to the P0 vertex.

2. Track-track VO's include any pair of oppositely charged tracks which decay in the volume between the target and P0. The tracks are required to have a distance of closest approach of less than 0.1 inch. This requirement is actually quite loose, but is consistent with the philosophy that one can always reject it at some later point since the distance of closest approach is saved along with all other pertinent information. 
The resulting VO's are further divided into two classes dedepending on whether both tracks are initially assigned to the event vertex (Case 6 VO's) or not (Case 2 VO's). Naturally those V0's with both tracks initially assigned have considerably worse signal to noise.

3. Track-stub V0's are the most difficult to recognize. The sample of track-stub pairs whose $\mathrm{xz}$ intersection is consistent with the same decay volume as for track-track VO's is selected. Because the momentum resolution for the stub depends on the $z$ resolution of this intersection, a minimum opening angle of $5 \mathrm{mr}$ is demanded in the $\mathrm{xz}$ view. Picking the momentum which swims the stub back to the $y$ of the track at the $\mathrm{xz}$ intersection allows one to calculate the VO's mass and position at the target. Again the V0's are flagged depending on whether both members were previously assigned to the production vertex (Case 7 Vo's) or not (Case $\left.3 \mathrm{VO}^{\prime} \mathrm{s}\right)$.

Figure 17 shows a typical sample of $\Lambda^{0}$ 's and $\bar{\Lambda}^{0}$ 's for cases $1,2,3$.

The V0 program also includes a complicated arbitration scheme to resolve track sharing. It is fairly common, particularly in track-stub Vo's, for one track to be a member of more than one VO. The arbitration is biased towards V0's that have masses consistent with $\mathrm{K}_{\mathrm{S}}^{0}$ 's or $\Lambda^{0}$ 's. If both V0's have masses within $5 \sigma$ of either the $\mathrm{K}_{\mathrm{S}}^{0}$ or $\Lambda^{0}$ they are passed to another level of arbitration. All tests are of a box nature, that is they are checked only for consistency with known experimental resolutions. V0's which are the result of arbitration are flagged with a warning indicating they were not unambiguously identified.

After all V0 candidates are identified, the vertex routine is recalled in an attempt to incorporate the Vo's into the total event topology. If a new vertex results, stub momenta are recomputed for the new vertex and all track assignments are redone. The topology of the charged tracks is now complete. 
CASE $1+4+5 \lambda^{\prime} \mathrm{s}$

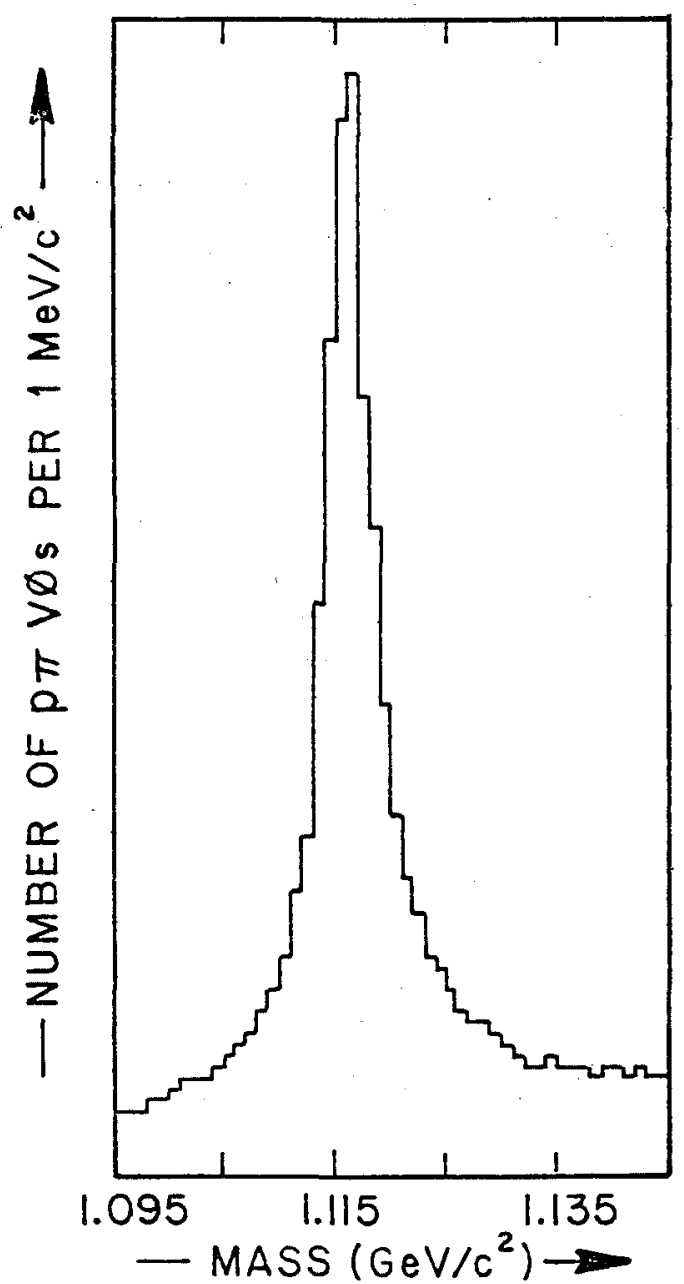

(a)
CASE $2 \lambda^{\prime}$ '

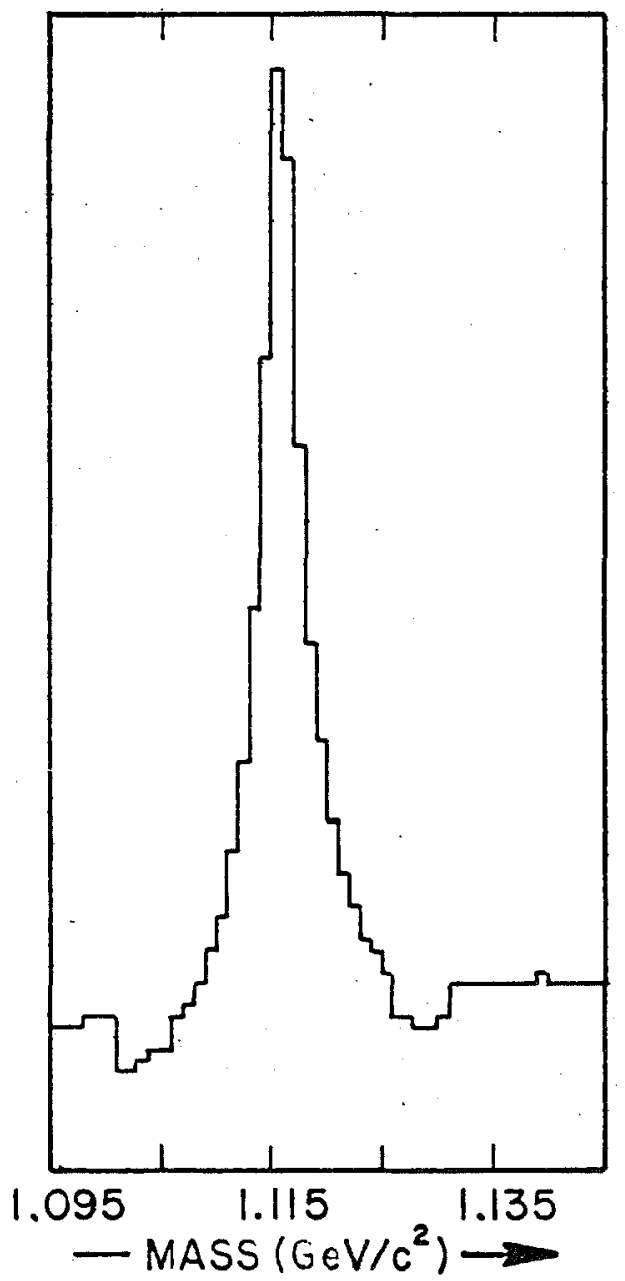

(b)
CASE $3 \lambda^{\prime}$ S

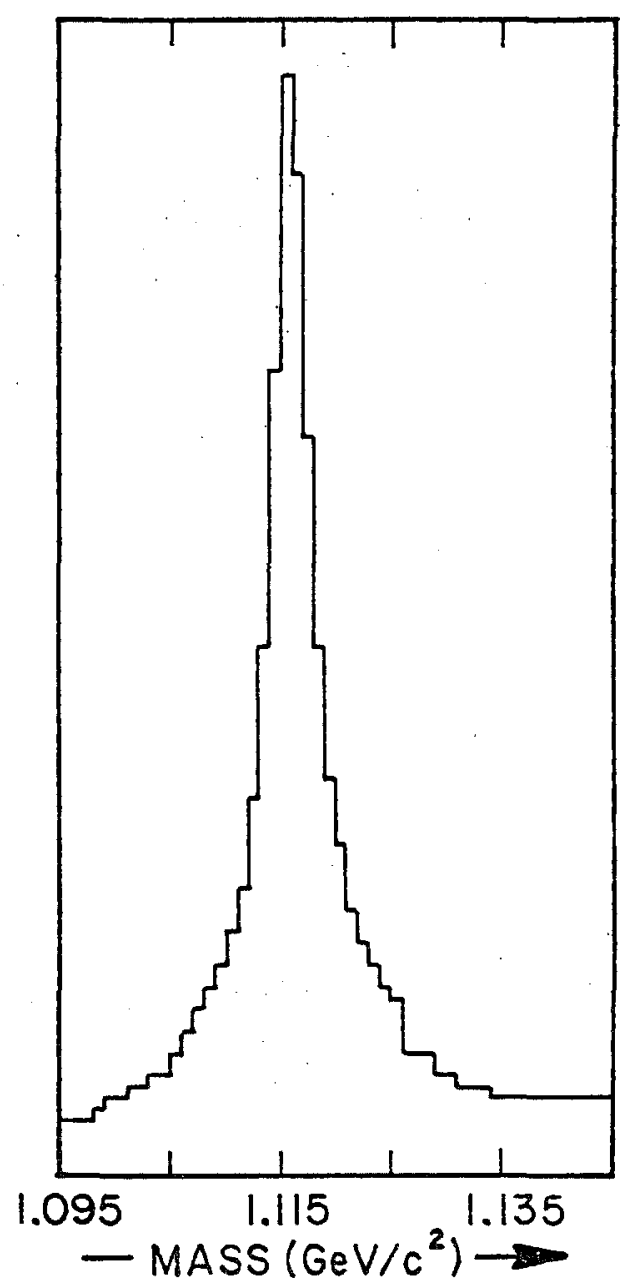

(c)

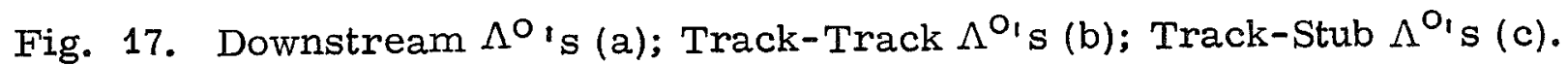




\section{Cherenkov Identification}

The Cherenkov analysis is now done on the charged tracks. The fraction of light from each track which would fall on each mirror is calculated. The initial fractions are based on the assumption that all tracks are electrons. This has the effect of producing the most light and therefore the most confusion possible in the counter. A complicated shifting procedure looks for tracks which have a significant amount of light predicted on a mirror which is off. Expected light yields are then recomputed with this new knowledge. Each track is then tagged with two numbers (one for $\mathrm{C} 1$, one for $\mathrm{C} 2$ ) indicating whether the track produced light (on), did not produce light (off), or was indeterminate (don't know). Table IV shows the translation of these codes in particle types.

The routine is biased towards producing a clean sample of kaons and protons. Because pions outnumber heavy particles more than twenty to one, one cannot tolerate the leakage of pions into the sample of heavy particles and, therefore, must be conservative and accept the loss of a percentage of heavy particles into the pion classes.

The actual performance of the Cherenkov counters is best studied by using the data. Figure 18 shows an unbiased sample of case $3 \Lambda^{0}$ 's (one track and one stub, at least one member not pointing at the production vertex). This sample is then broken down into three categories according to the cherenkov identification of the high momentum particle. Approximately $75 \%$ 
TABLE IV

CHERENKOV IDENTIFICATION TABLE

\begin{tabular}{|c|c|c|c|c|c|c|c|c|c|}
\hline $\mathrm{C} 2$ & \multicolumn{3}{|c|}{ OFF } & \multicolumn{3}{|c|}{ ON } & \multicolumn{3}{|c|}{ DON'T KNOW } \\
\hline$\frac{C 1}{00=}$ & OFF & ON & $\begin{array}{l}\text { DON'T } \\
\text { KNOW } \\
\end{array}$ & OFF & ON & $\begin{array}{l}\text { DON'T } \\
\text { KNOW }\end{array}$ & OFF & ON & \begin{tabular}{|l} 
DON'T \\
KNOW' \\
\end{tabular} \\
\hline & $\pi / \mathrm{K} / \mathrm{p}$ & $?$ & $\pi / \mathrm{K} / \mathrm{p}$ & $?^{*}$ & e & e & $\pi / \mathrm{K} / \mathrm{p}$ & e & $?$ \\
\hline & $K / p$ & $\pi$ & $\pi / \mathrm{K} / \mathrm{p}$ & $?^{*}$ & e & e & $K / p$ & $\pi$ & ? \\
\hline & $K / p$ & $\pi^{*}$ & $K / p$ & $\pi^{*}$ & $\pi$ & $\pi$ & $K / p$ & $\pi$ & ? \\
\hline & p & $K$ & $K / p$ & $\pi^{*}$ & $\pi$ & $\pi$ & $p$ & $\pi / \mathrm{K}$ & $?$ \\
\hline & $p$ & $\mathrm{~K}$ & $K / p$ & $\pi / K^{*}$ & $\pi / K$ & $\pi / K$ & $p$ & $\pi / K$ & $?$ \\
\hline & p & p & P & $\pi / K^{*}$ & $\pi / \mathrm{K}$ & $\pi / K$ & $?$ & $?$ & $?$ \\
\hline
\end{tabular}




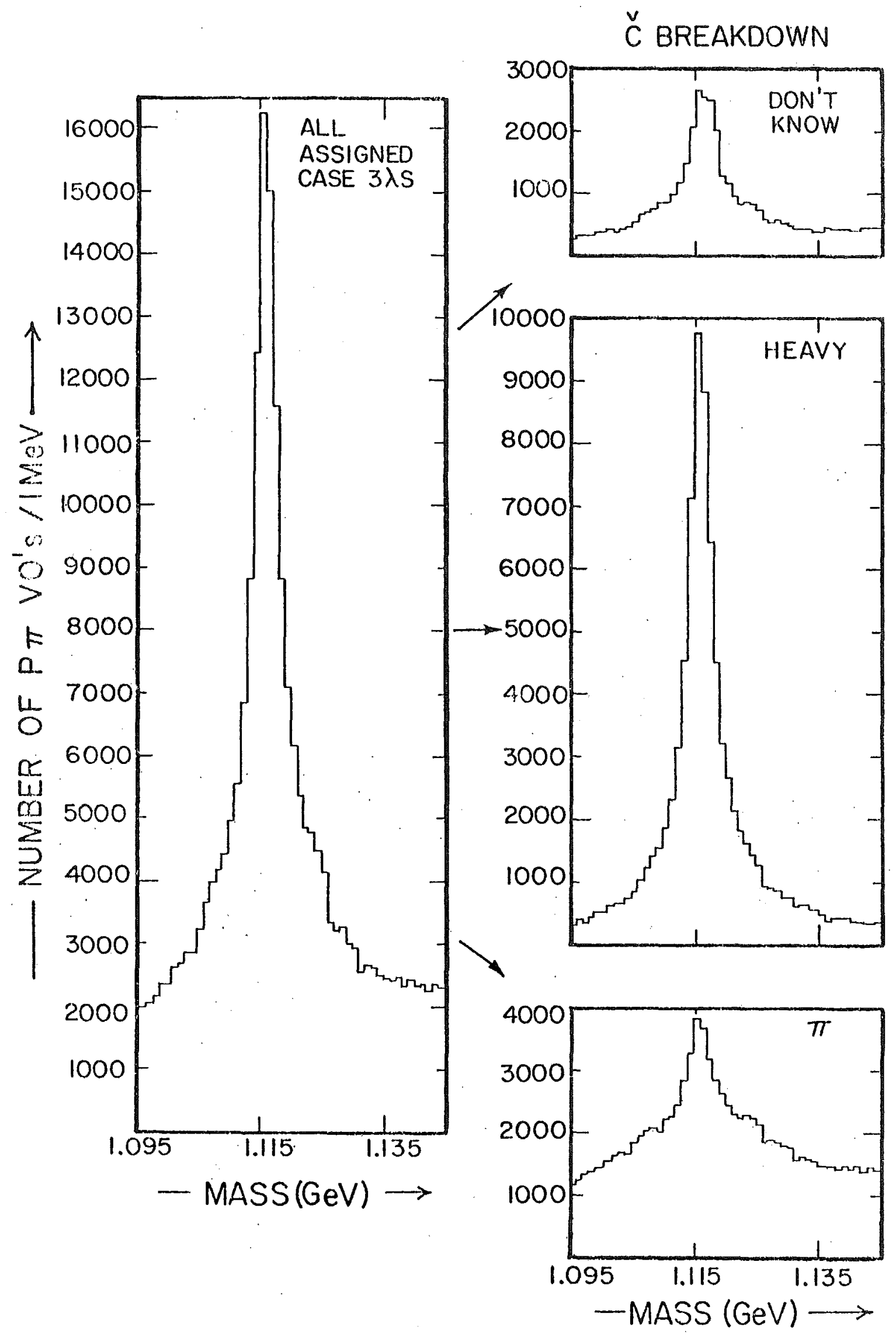

Fig. 18. Cherenkov Breakdown of Case. $3 \Lambda^{\mathrm{O}_{1}} \mathrm{~s}$. 
of the $\Lambda^{0}$ 's correctly have their high momentum track identified as being inconsistent with a pion. The Cherenkov iıformation on $22 \%$ of the $\Lambda^{0}$ 's is confused leading to the "don's know" category. The final plot shows that $2.5 \%$ of the $\Lambda^{0}$ 's have their high-momentum track misidentified as a pion.

The contamination of the heavy-particle sample can likewise be studied using a clean sample of $\mathrm{K}_{\mathrm{S}}^{0} \mathrm{~s}$, where one is guaranteed both particles are $\pi$ 's. Figure 19 shows the same breakdown by Cherenkov counter information for the case $2 \mathrm{~K}_{\mathrm{S}}^{0}$ 's (don't know, heavy, pion). The contamination of the $\pi$ 's that have been misidentified as heavy particles is about 2.5\%. Using the $\mathrm{K}_{S}^{0}$ 's and $\Lambda^{0}$ 's is an effective way of obtaining the desired balance of the signal/noise properties in the Cherenkov counter algorithms. The true power of the Cherenkov counters is best seen by the extraction of a clean case 6 (both tracks initially assigned to the production vertex) $\Lambda^{0}$ signal (Fig. 20). The raw plot shows a very poor signal/noise and the arbitration box characteristic of VO's in which the pattern recognition has been confused. The background and arbitration box are eliminated by demanding the high-momentum track be inconsistent with a $\pi$.

The effectiveness of each counter was reduced by several problems. Although C1's physical environment inside $M 2$ necessitated large amounts of shielding and bucking coils, the real problem occurs at the analysis level. The bending particles made computing the expected fraction of light on each mirror a difficult task. A low-momentum particle will spray its light around the counter as it bends, adding to the confusion and effectively 


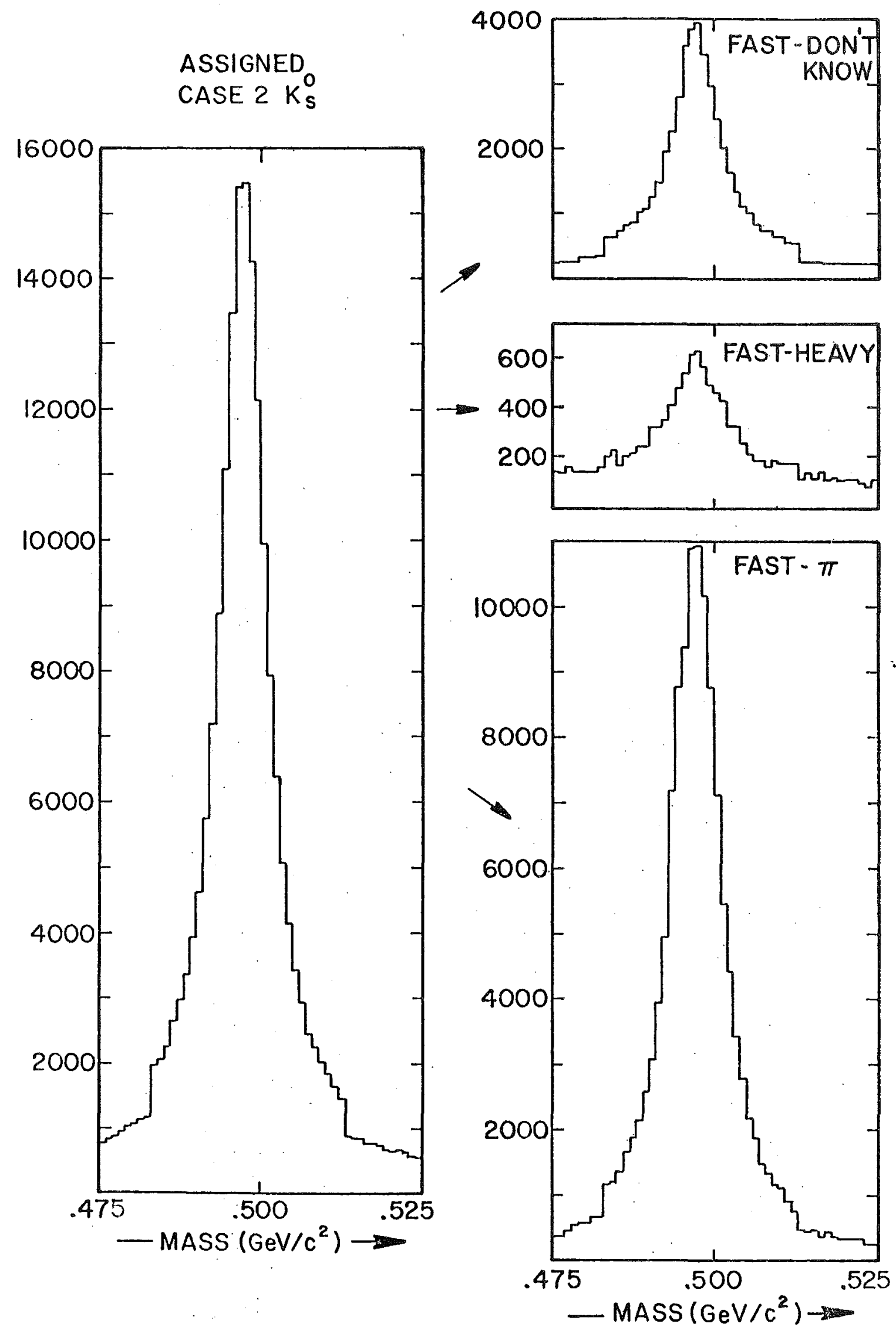

Fig. 19. Cherenkov Breakdown of Case $2 \mathrm{~K}_{\mathrm{S}}{ }^{\mathrm{O}_{1}} \mathrm{~s}$. 


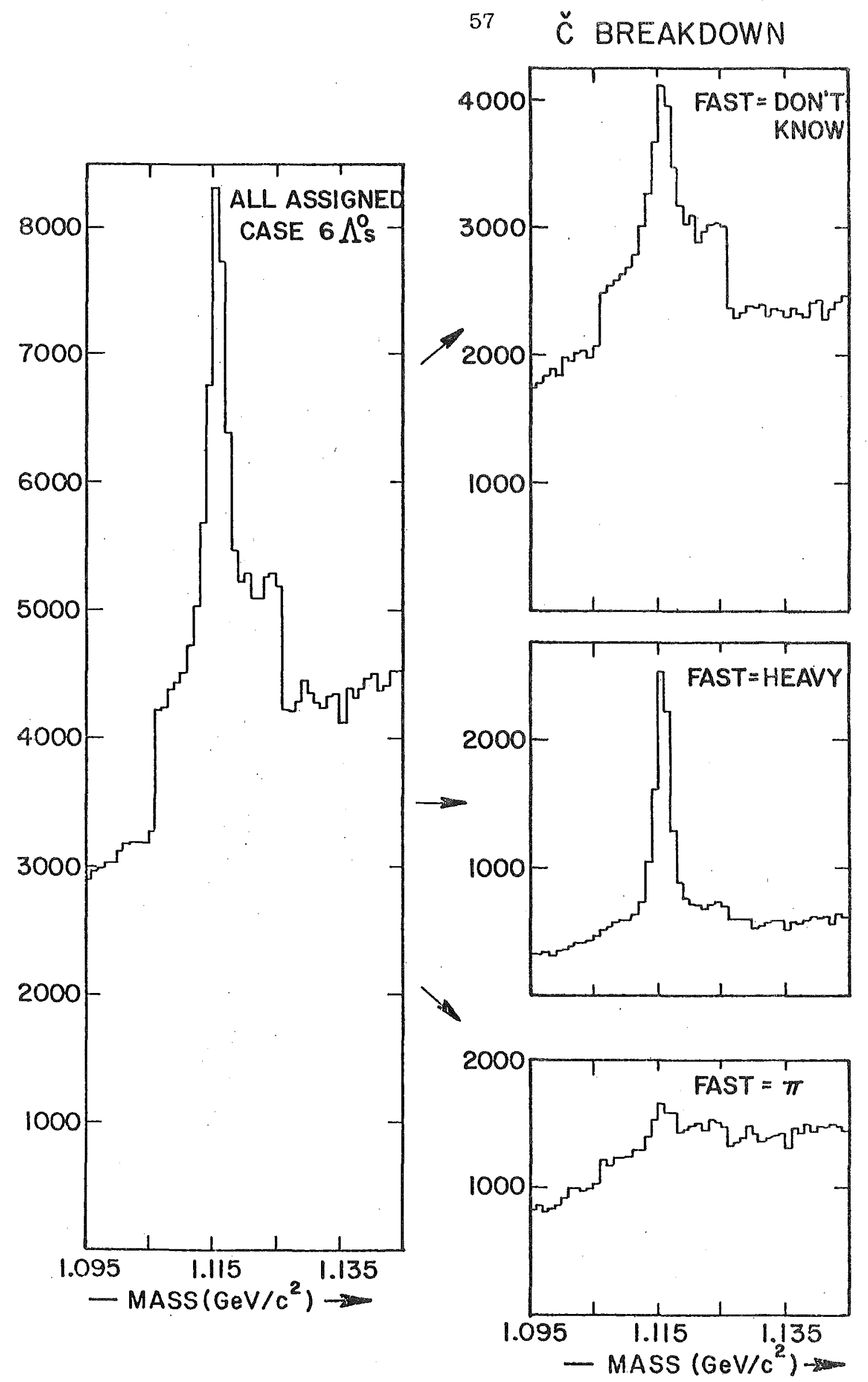

Fig. 20. Cherenkov Breakdown of Case $6 \Lambda^{\mathrm{O}^{\mathrm{l}}} \mathrm{s}$. 
decreasing the granularity of the system. In addition, stubs which terminate their path within $\mathrm{C1}$ cause indeterminate results. C2!'s problem was more hardware-oriented. An $\mathrm{e}^{+} \mathrm{e}^{-}$pair occasionally passed directly through a phototube window of $\mathrm{C} 2$. If the apparatus was triggered before the phototube could recover from this event, bogus light was registered. Of course, if no track points to a mirror that is on, there is no problem. This, however, is not always the case and occasionally the extra light will confuse the pattern recognition process in $\mathrm{C} 2$ resulting in an inefficiency for finding heavy particles. The problem is mainly confined to the central mirrors.

\section{Event Selection}

Finally events are tagged and classified by type to facilitate the editing of a particular final state. Loose definitions flagged events with any $V 0$, a $K_{S}^{0}$, a $\Lambda^{0}$, one heavy particle, two heavy particles, a diffractive event, one or two muons, etc. Selecting a sample of interest is, computerwise, very economical and fast, usually only requiring a bit match.

The final states of interest here are characterized by containing at least one of the following:

1.
2. Proton- $-\mathrm{K}_{\mathrm{S}}^{0}$
3.
$\Lambda^{0}$

The final data selection consisted of three edits on the 200 four-vector tapes. This produced three tapes with a vo within $\pm 30 \mathrm{MeV} / \mathrm{c}^{2}$ of the $\mathrm{K}_{\mathrm{S}}^{0}$ and a cleanly identified proton $(84,000$ events). The requirement of a V0 within $\pm 30 \mathrm{MeV} / \mathrm{c}^{2}$ of the $\Lambda^{0}$ mass yields seven tapes (about 1 million events). Similarly 
there are three tapes (about 360,000 events) with at least one unambiguous proton and a second heavy particle. 


\section{CHARM BARYON SEARCH}

The lowest lying charm baryon is the quark combination cud, alternately called the $\Lambda_{\mathrm{c}}^{+}$or $\mathrm{C}_{0}^{+}$. As stated in Chapter $\mathrm{I}$, this state is stable against strong decays provided its mass is below $2.8 \mathrm{GeV} / \mathrm{c}^{2}$ (the kinematic limit for $\Lambda_{\mathrm{c}}^{+} \rightarrow \mathrm{pD}^{0}$ ). Although a myriad of Cabbibo allowed weak decays are possible, the modes to which this experiment is most sensitive are

$$
\begin{array}{ll}
\text { 1. } & \Lambda^{0} \pi \\
\text { 2. } & \Lambda^{0} \pi \pi \pi \\
\text { 3. } & \mathrm{pK}^{\mathrm{K}} \\
\text { 4. } & \mathrm{pK}_{\mathrm{S}}^{\mathrm{O}}
\end{array}
$$

The ability to observe the $\Lambda_{c}^{+}$decaying into one of these final states depends on several unrelated factors:

1. The branching ratio into the state

2. The experimental detection efficiency

3. The backgrounds encountered.

A description detailing the mechanics involved in analyzing each of these final states is presented in Sections A-C. Section D discusses the Monte Carlo and the model used to extract the detection efficiencies for each of these channels. Finally a summary of the results is given in section E. The cross section times branching ratio for $\mathrm{pK}^{0}$ and upper limits for the remaining three channels are extracted.

A. $\Lambda^{0} \pi$ and $\Lambda^{0} \pi \pi \pi$

A11 events having a VO candidate with a pr mass within \pm 30 $\mathrm{MeV} / \mathrm{c}^{2}$ of the $\Lambda^{0}$ mass are selected. The signal to background obtained from the pattern recognition alone is unacceptable. The wide mass cut used in editing the sample allows study of the background beneath the $\Lambda^{0}$ peak. The clean-up procedure used to 
reduce the noise to an acceptable level is different for each of the seven categories of Vo's found. Three main criteria are used.

1. All $\Lambda^{0}$ 's are required to be assigned to the target (within 0.13 inch). This is a rather generous cut, but, nevertheless, reduces the background for downstream VO's to an acceptable level. As the decay vertex of the vo moves closer to the target, this cut becomes less effective.

2. If the fast particle is identified as either a proton or is ambiguous between proton and kaon, the $\Lambda^{0}$ is accepted. Conversely, if the fast particle is identified as a $\pi$, it is rejected. Figure 21 shows the effect of these requirements on a sample of Case $3 \Lambda^{0}$ 's (track-stub, at least one member not assigned to the primary vertex). This sample is broken into three subsamples depending on the Cherenkov identification of the faster member of the V0. The proton requirement is seen to be very effective in reducing the background under the $\Lambda^{0}$ peak (Fig. 21b). The corresponding $\pi$ cut (Fig. 21c) costs one some of the $\Lambda^{0}$ signal as expected because of the philosophy employed in the Cherenkov counter routine. For VO's with both member tracks assigned (Case 6 and 7), the fast track must be inconsistent with a $\pi$.

3. The remaining $\Lambda^{0}$ candidates (Fig. 21a) have no useful Cherenkov information. Tighter requirements on the topology of the VO are therefore demanded. If the distance of closest approach of the V0 to the event vertex if less than 0.04 inches, it is accepted. For track-track VO's (Cases 2 and 6) the distance of closest approach of the two tracks is required to be less than 0.03 inches if the tighter vertex cut fails. In addition, all $\Lambda^{0}$ candidates with no Cherenkov information are required to have their $\pi^{+} \pi^{-}$mass inconsistent with the $\mathrm{K}_{\mathrm{S}}^{0}$ mass (not within $\pm 15 \mathrm{MeV} / \mathrm{c}^{2}$ ).

Adjustment of the various cut parameters can produce an arbitrarily good signal to noise but at the expense of signal. As will be seen, the background in the mass plots is primarily due to events containing a real $\Lambda^{0}$. Consequently the usefulness of this approach diminishes rapidly as the requirements are tightened. The criteria used here are about 90\% efficient (i.e., $10 \%$ of the geometrically accepted and found $\Lambda^{0}$ 's are rejected). 


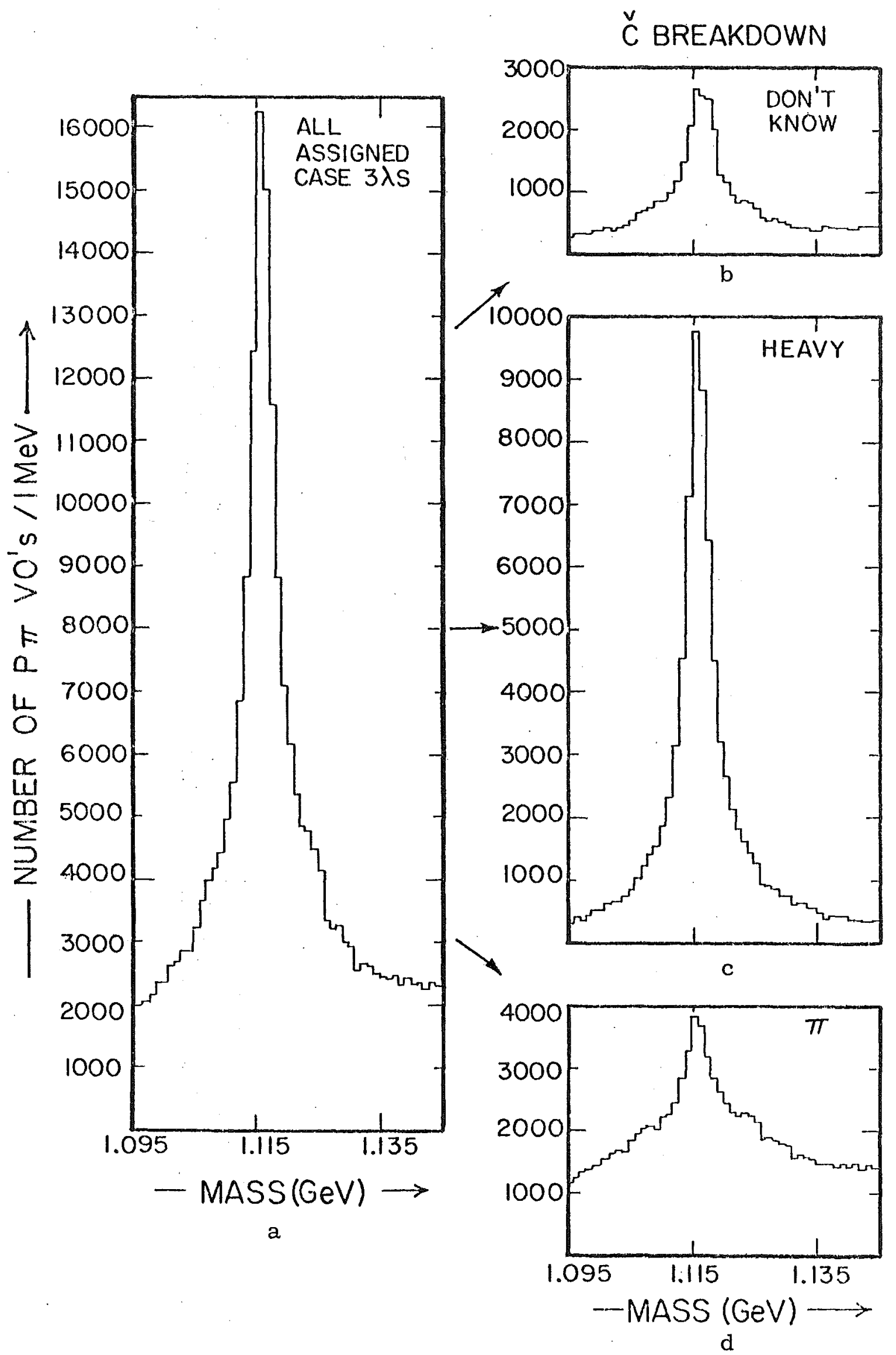

Fig. 21. Cherenkov Breakdown of Case $3 \Lambda^{0}, \mathrm{~s}$. 
Figure $22 \mathrm{a}$ shows all $\Lambda^{0}$ 's candidates identified by pattern recognition. Figure $22 \mathrm{~b}, \mathrm{c}$ shows the accepted and rejected $\Lambda^{0}$ 's respectively. A final mass cut requires that the $\Lambda^{0}$ candidate be consistent with the resolution. This cut varies from \pm 3 to \pm 7 $\mathrm{MeV} / \mathrm{c}^{2}$ around the physical $\Lambda^{0}$ mass $\left(1.1156 \mathrm{GeV} / \mathrm{c}^{2}\right)$, depending on the $z$ of its decay. The member tracks of rejected $\Lambda^{0}$ candidates are treated as any other track in the event.

The $\pi$ 's allowed to enter in the mass combination are required only to be assigned to the primary event vertex and have a Cherenkov identification consistent with a $\pi$. Only events within \pm 4.0 inches of the target's midplane are considered. The $z$ distribution of a typical sample of events was shown in Fig. 12a. The number of tracks allowed opposite the potential $\Lambda_{\mathrm{c}}$ combination is restricted to fall between two and six, commensurate with the multiplicities expected for the decay of another $\Lambda_{c}^{-}$. In searching for the decay $\Lambda_{c}^{+}+\Lambda^{0} \pi \pi_{\pi}^{+}{ }_{\pi}^{-}$the maximum allowed number of recoiling tracks is reduced to five in an attempt to control the rapid increase in combinatorial groupings. The upper limit is chosen by considering the worst case situation of a recoiling $\Lambda_{C}^{-} \rightarrow \bar{\Lambda}_{\pi}^{0}+{ }_{\pi}-$ decay (counted as four tracks) plus two additional $\pi$ 's if the $\Lambda_{c}$ 's are the result of $\Sigma_{c}$ 's cascading to $\Lambda_{c}$ 's.

The mass plot for $\Lambda^{0} \pi \pi \pi$ is subdivided into four categories corresponding to the correct and incorrect strangeness-charge correlation for the baryon (Fig. 23a,b) and anti-baryon (Fig. $24 \mathrm{a}, \mathrm{b})$ channels. To estimate the number of background entries (not from the photons) the same plots are generated for the $\mathrm{K}^{0} \mathrm{~L}$ 
64

$\times 1000$

$\times 1000$
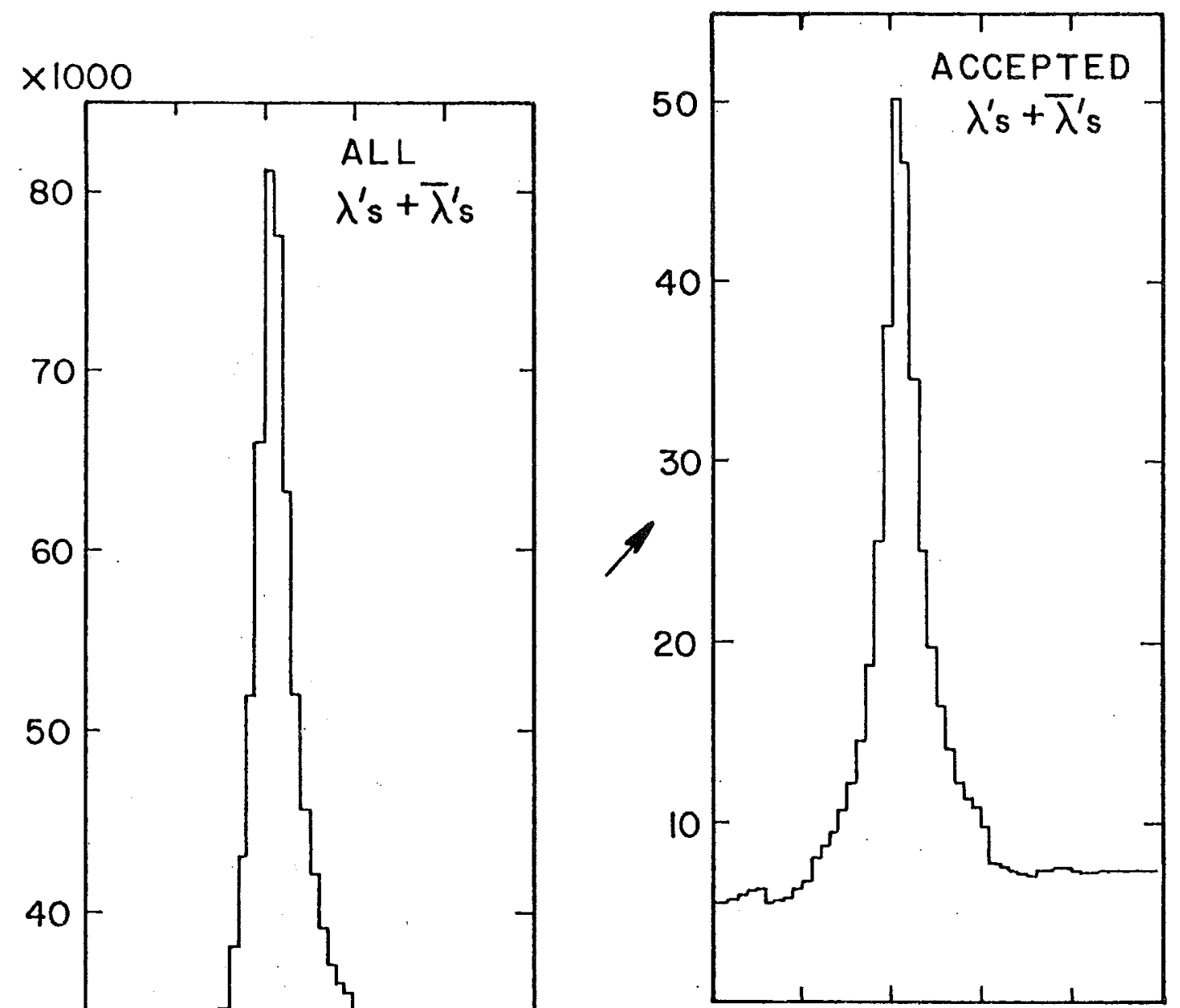

(b)

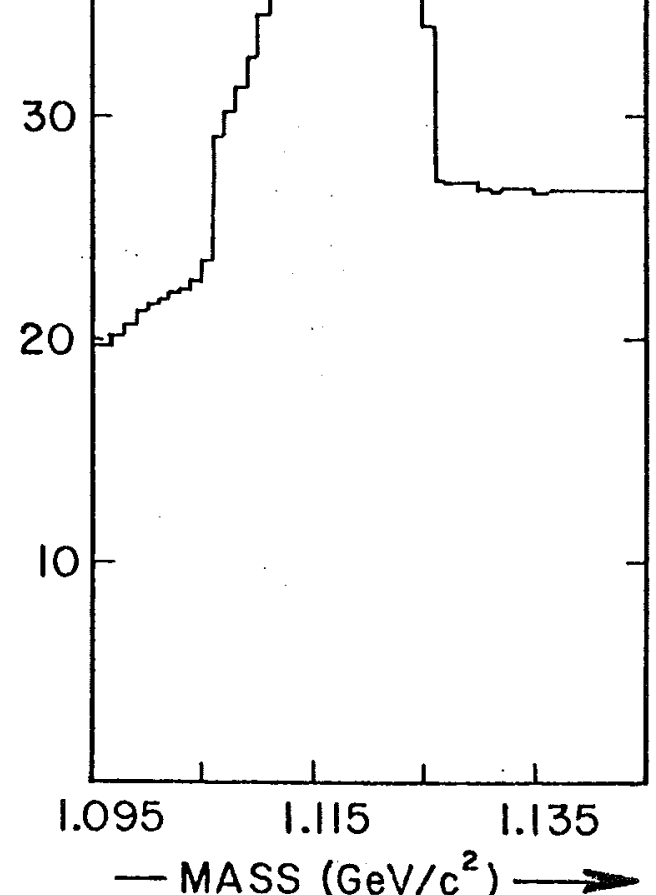

(a)

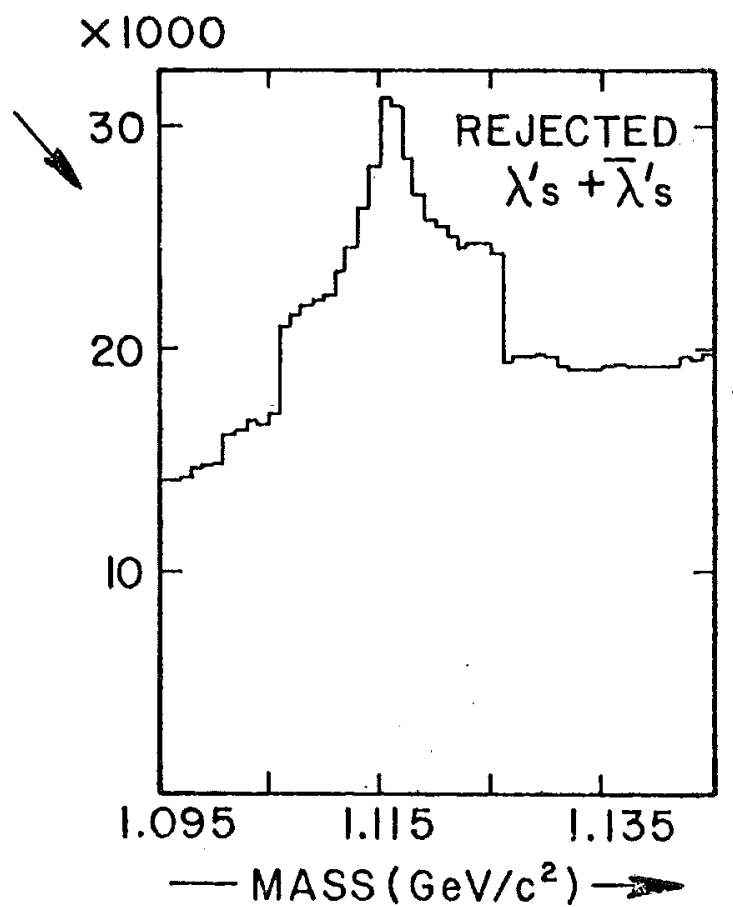

(c)

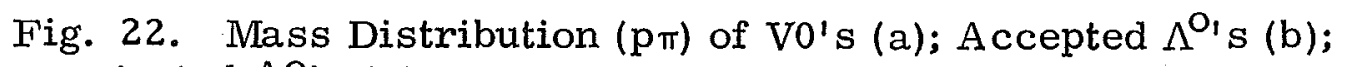
Rejected $\Lambda^{\mathrm{O}_{\mathrm{S}} \mathrm{s}}$ (c). 

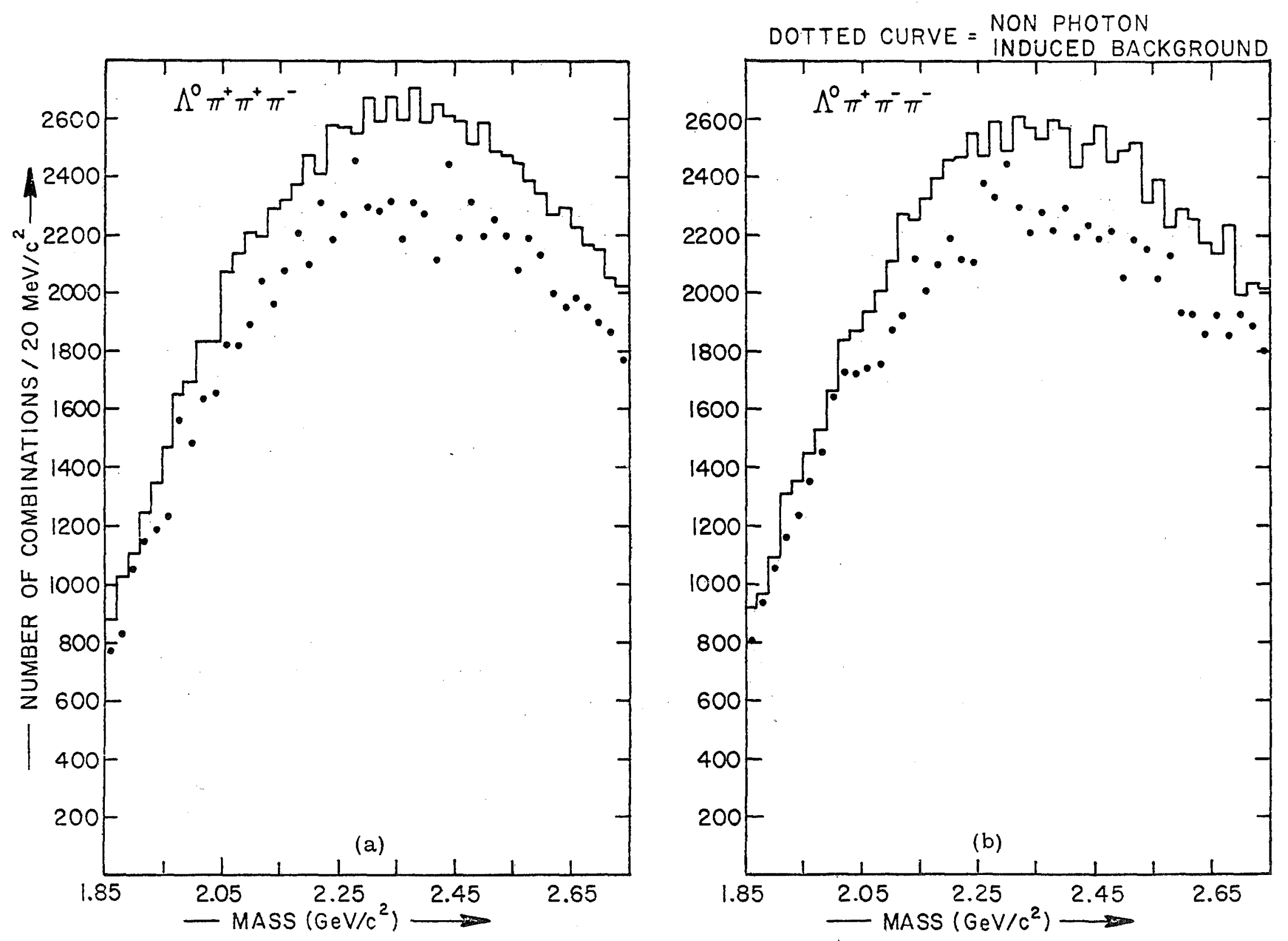

Fig. 23. Mass Distribution of $\Lambda^{\circ} \pi^{+} \pi^{+} \pi^{-}$(a) and $\Lambda^{\circ} \pi^{+} \pi^{-} \pi^{-}$(b). 

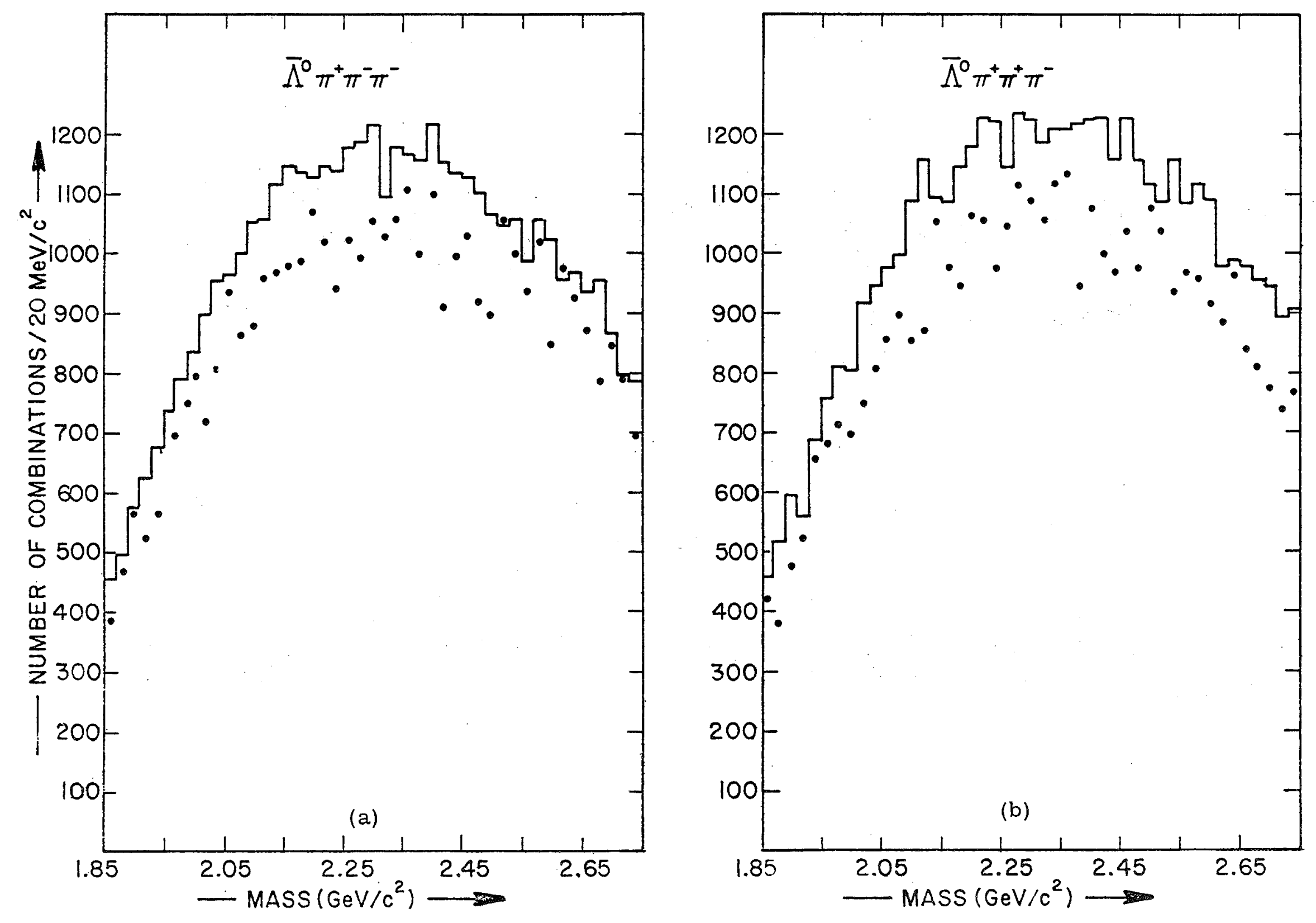

Fig. 24. Mass Distribution of $\Lambda^{\circ} \pi^{+} \pi^{-} \pi^{-}$(a) and $\Lambda^{0} \pi^{+} \pi^{+} \pi^{-}$(b). 
runs, represented by the dotted curves in the same figures. (A scale factor of 2.35 is needed to equalize the relative flux for the two samples.) These curves show that $80-9.0 \%$ of the entries are not photon induced.

The four $\Lambda^{0} \pi$ plots are shown in Figs. $25 \mathrm{a}, \mathrm{b}$ and $26 \mathrm{a}, \mathrm{b}$ together with the four non-photon induced plots (dotted curves). Even though the "photon" data is still 80-90\% nonphoton induced, there is a drastic reduction in the number of entries in the $\Lambda^{0} \pi$ plots compared to the $\Lambda^{0} \pi \pi \pi$ plots, thus increasing the sensitivity of this mode. Comparing the $\Lambda^{0} \pi^{+}$ (Fig. 25a) with the $\Lambda^{0} \pi^{+} \pi^{-}-$plot (Fig. 23a) in the mass region near $2.275 \mathrm{GeV} / \mathrm{c}^{2}$ shows that there are about 500 entries per 20 $\mathrm{MeV} / \mathrm{c}^{2}$ bin for $\Lambda^{0} \pi$ against 2400 for $\Lambda^{0}{ }_{\pi}{ }_{\pi}+_{\pi}{ }^{-}$. The reason for this large difference is twofold. The peak of the $\Lambda^{0} \pi \pi_{\pi}+^{-}$mass distribution is near the expected $\Lambda_{c}^{+}$mass, whereas the two-body $\Lambda^{-}+$distribution peaks at $1.4 \mathrm{GeV} / \mathrm{c}^{2}$ and falls of nearly exponentially with increasing mass. The combinatorial background is also much larger for the $\Lambda^{0} \pi_{\pi}{ }^{+}{ }_{\pi}^{-}$. A typical eight-track event will result in three $\Lambda^{0} \pi^{+}$combinations (assuming one $\Lambda^{0}$ per event counting as two tracks leaving six $\pi$ candidates, only half of which give the correct strangeness-charge correlation). The same eight-track event taken as $\Lambda^{0} \pi^{+} \pi^{+} \pi^{-}$enters an average of nine times $(3(3 ! / 2 !))$.

There is no evidence for the $\Lambda_{c}^{+}$decaying through either of these two channels. The limits on $\sigma B$ for both of these channels are given in Section E. 

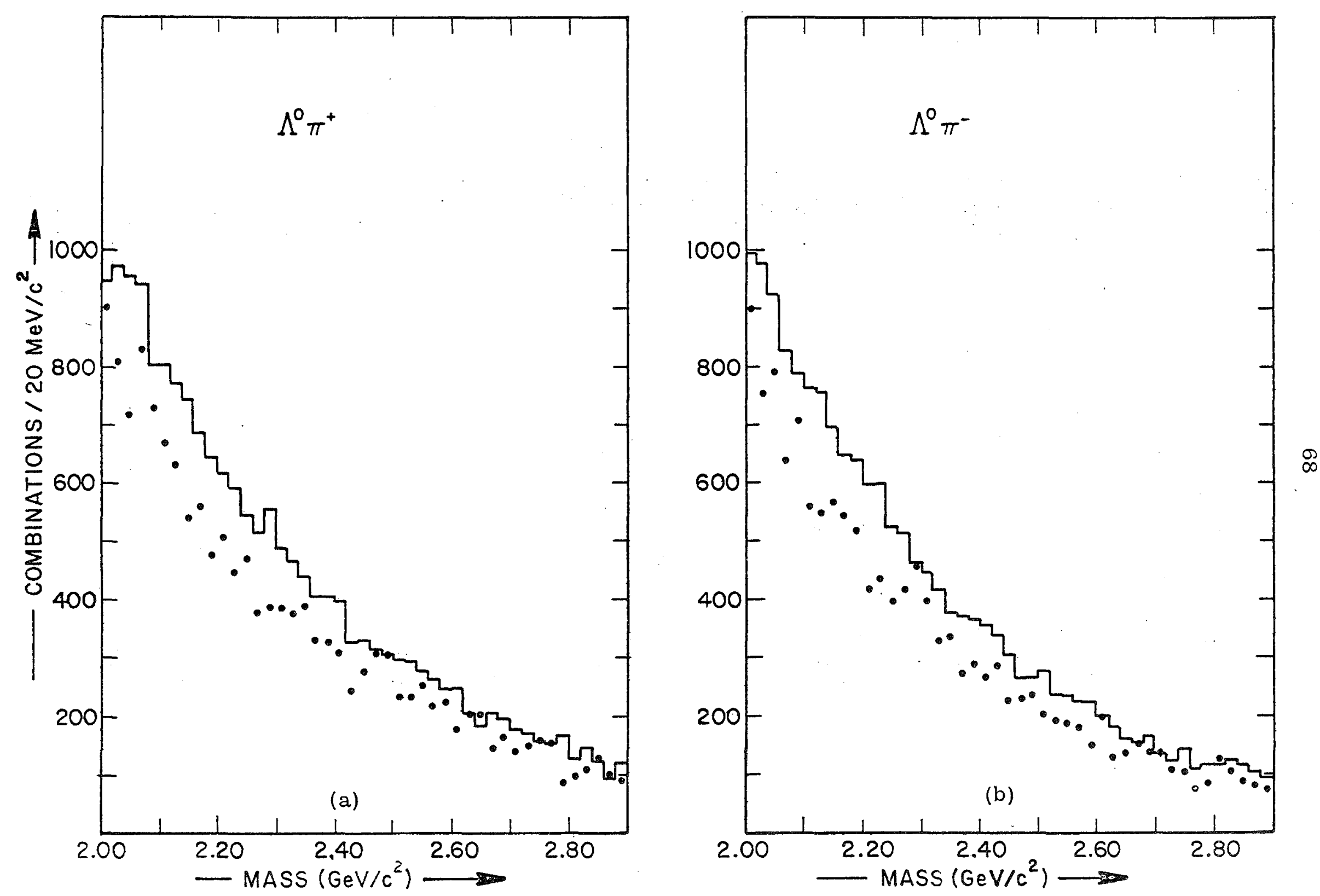

Fig. 25. Mass Distribution of $\Lambda^{\circ}{ }^{+}(a)$ and $\Lambda^{\circ} \pi^{-}(b)$. 

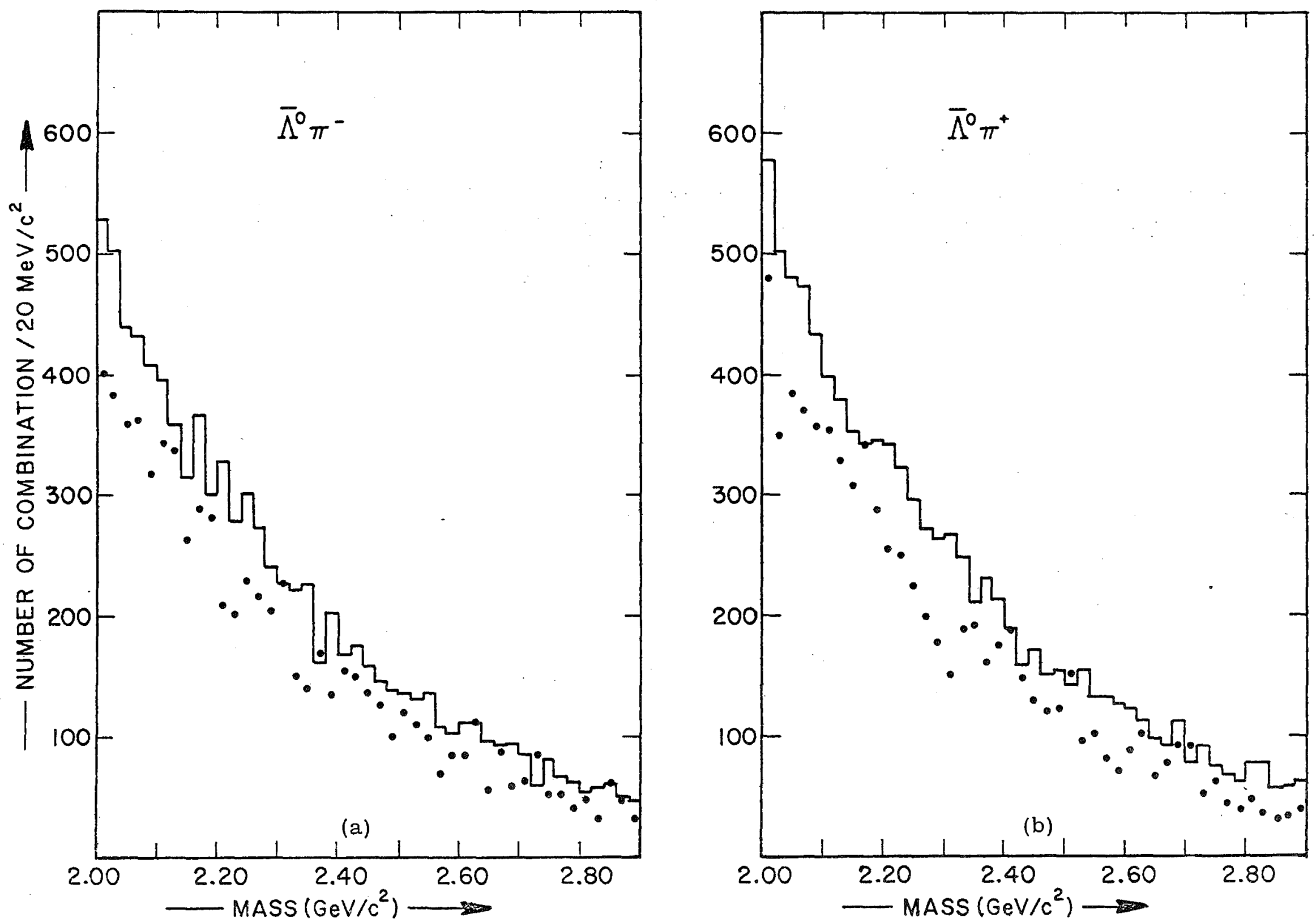

8

Fig. 26. Mass Distribution of $\bar{\Lambda}^{\circ} \pi^{-}(a)$ and $\bar{\Lambda}^{\circ} \pi^{+}(b)$. 
B. $\mathrm{pK} \pi$

All events with a cleanly identified proton and another charged particle identified as a kaon or kaon/proton ambiguous are selected. The vertex, multiplicity, and $\pi$ requirements are as in the $\Lambda^{0}$ channels. Limiting the kaon candidates to only positively identified cases cuts the acceptance to an unacceptable level. This occurs because a clean kaon identification requires not only the kaon to be in the proper momentum range (20-40 GeV/c) but also information from both Cherenkov counters ( an "on" in C1 and an "off" in C2, see Table IV).

The high multiplicities of these events often confuses at least one of the counters. The data indicate that this is not a serious source of backgrounds, where the kaon-to-proton ratio is seen to be about five to one. (A word of caution is necessary though. This particle ratio is determined for the events in general and may not be true when applied to a highly specialized sample such as this, particularly when there is already one proton in the event.) The same series of four plots (baryon, anti-baryon for right and wrong strangeness-charge correlation) for the photon together with the non-photon induced background are presented in Figs. 27a,b and 28a,b. No signal is observed in either baryon or anti-baryon channel. The upper limit on oB for this channel is derived in section E.

C. $\quad \mathrm{pK}_{\mathrm{S}}^{0}$

The event selection criteria chooses any event which has a cleanly identified proton and a vo candidate with a $\pi^{+}{ }^{-}$mass within $\pm 30 \mathrm{MeV} / \mathrm{c}^{2}$ of the $\mathrm{K}_{\mathrm{s}}{ }^{0}$ mass. The cleanup procedure for the 

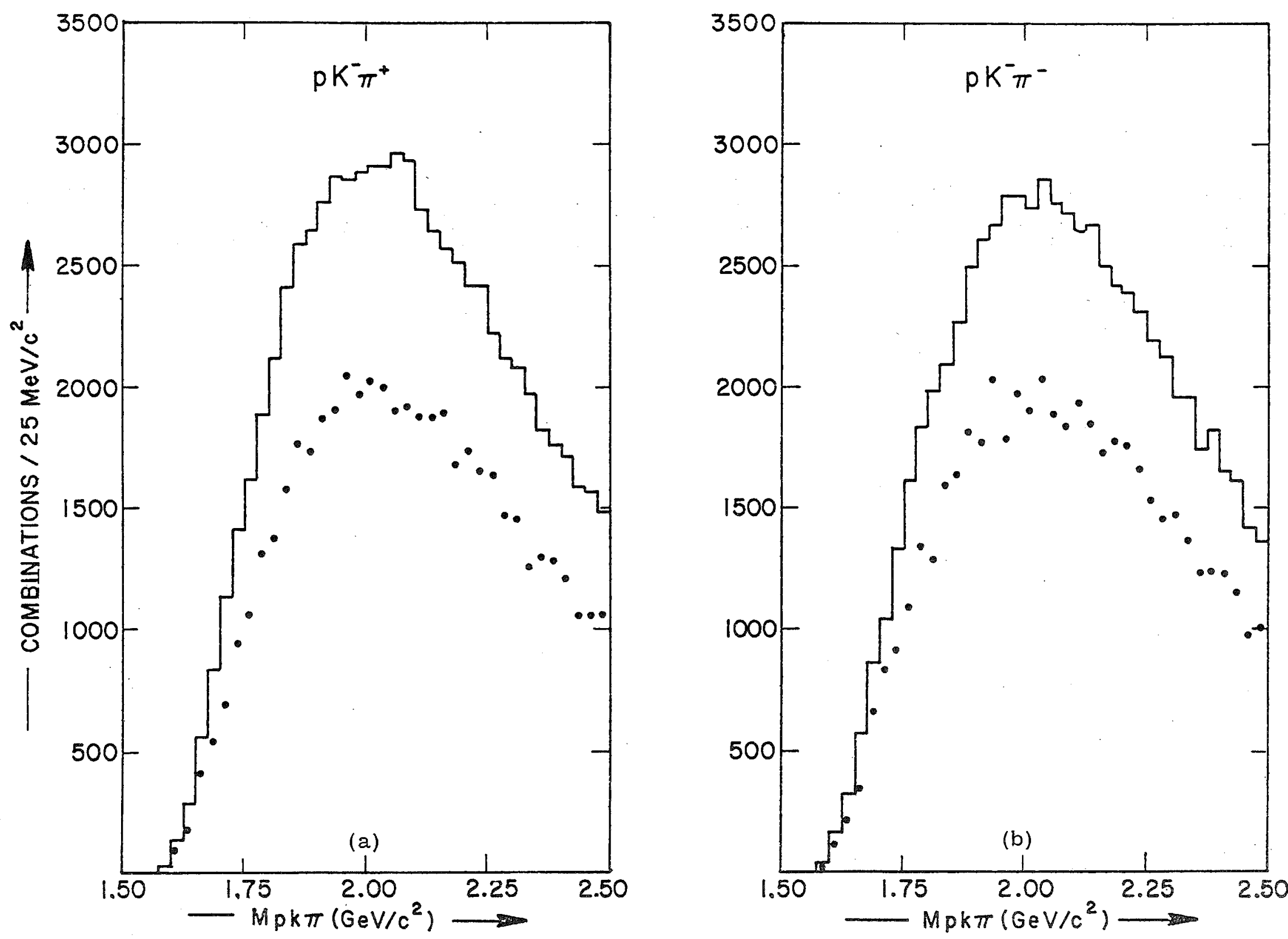

Fig. 27. Mass Distribution of $\mathrm{pK}^{-} \pi^{+}$(a) and $\mathrm{pK}^{+} \pi^{-}$(b). 


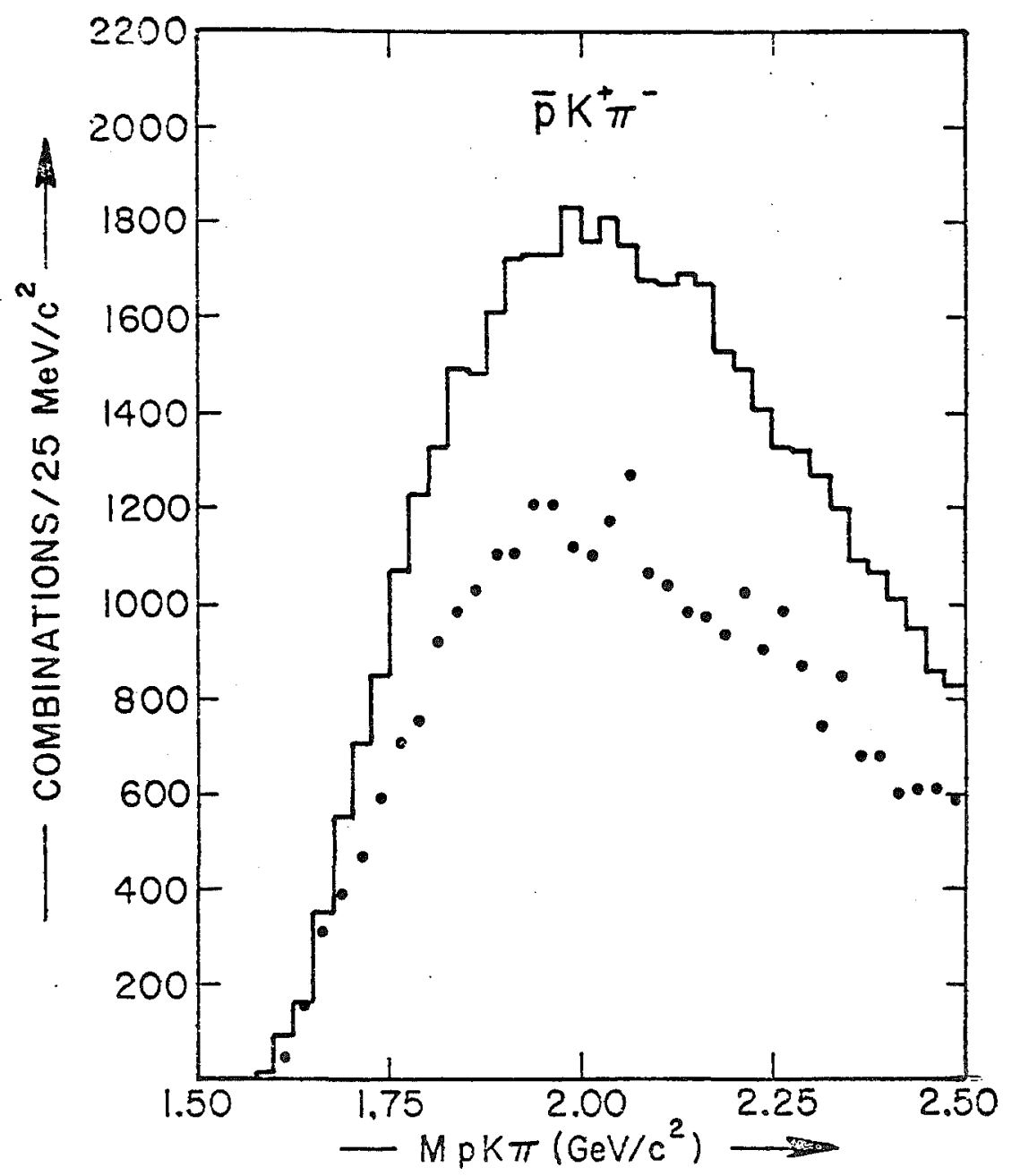

a

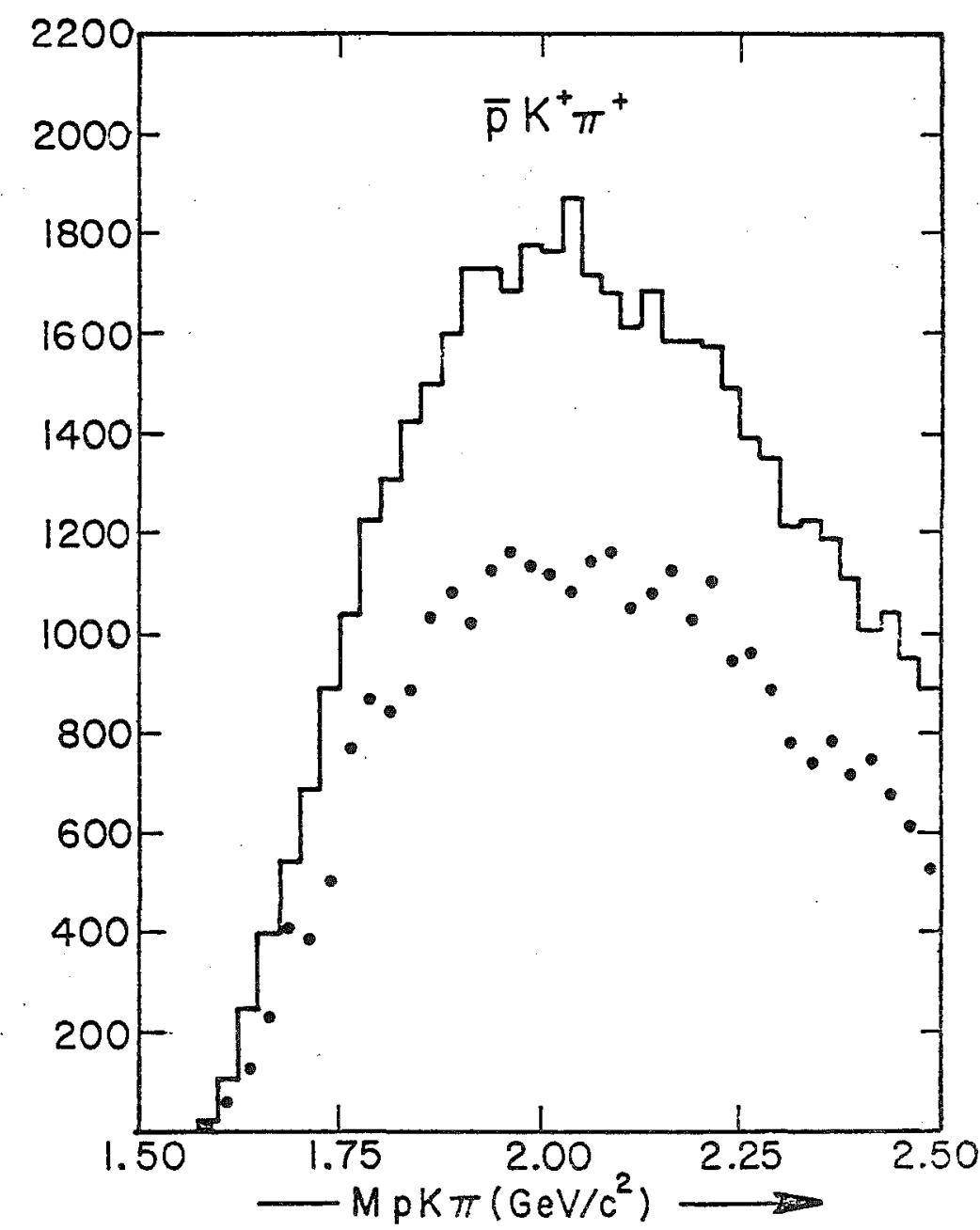

b

Fig. 28. Mass Distribution of $\overline{\mathrm{pK}}^{+} \pi^{-}$(a) and $\overline{\mathrm{pK}}^{-} \pi^{+}$(b). 
$\mathrm{K}_{\mathrm{S}}^{0}$ follows that of the $\Lambda^{0}$ 's with the obvious change of the proton requirement. The corresponding hard $\pi$ requirement is not nearly as strong and one is forced to reject $\mathrm{K}_{\mathrm{s}}^{0}$ 's with both member tracks pointing at the vertex as unacceptably noisy. After the $\mathrm{K}_{\mathrm{S}}^{0}$ 's have been properly laundered, a final mass cut of $\pm 15 \mathrm{MeV} / \mathrm{c}^{2}$ is applied. Figure 29 shows the subsample of $\mathrm{K}_{\mathrm{S}}{ }^{0}$ 's from the originally selected 84,000 events which survive these requirements. The member tracks of the rejected $\mathrm{K}_{\mathrm{s}}^{0}$ candidates become available for use as $\pi$ 's.

The requirement of a clean identification of the proton does not involve the same high cost in detection efficiency as did demanding the same of the kaon. Only one counter is required and the allowable momentum region is wider, from $\mathrm{C1}$ 's kaon threshold to C2's proton threshold $(20-80 \mathrm{GeV} / \mathrm{c}$, see Table IV) compared to 20-40 GeV/c for hard kaon. Since, as pointed out previously, the proton-to-kaon ratio in the ambiguous category is one to five, including these events will result in a similar rise in the background.

Using the criteria of high experimental detection efficiency, good signal-to-background ratio, and minimal combinatorial background, the $\Lambda_{\mathrm{c}}^{+}+\mathrm{p} \overline{\mathrm{K}^{0}}$ decay mode is this experiment's most sensitive mode provided that the $\Lambda_{c}^{+}$has a branching ratio to $\mathrm{p}^{0}$ roughly equal or greater than that of the other modes considered. The two-body, $\mathrm{pK}_{\mathrm{S}}^{0}$ phase space distribution peaks at 1.5 $\mathrm{GeV} / \mathrm{c}^{2}$ and falls quickly with mass as did the $\Lambda^{0} \pi$. The combinatorial problem is almost non-existent in this state. A typical event in, this sample has only one proton and one $\mathrm{K}_{\mathrm{S}}{ }^{0}$ resulting in 


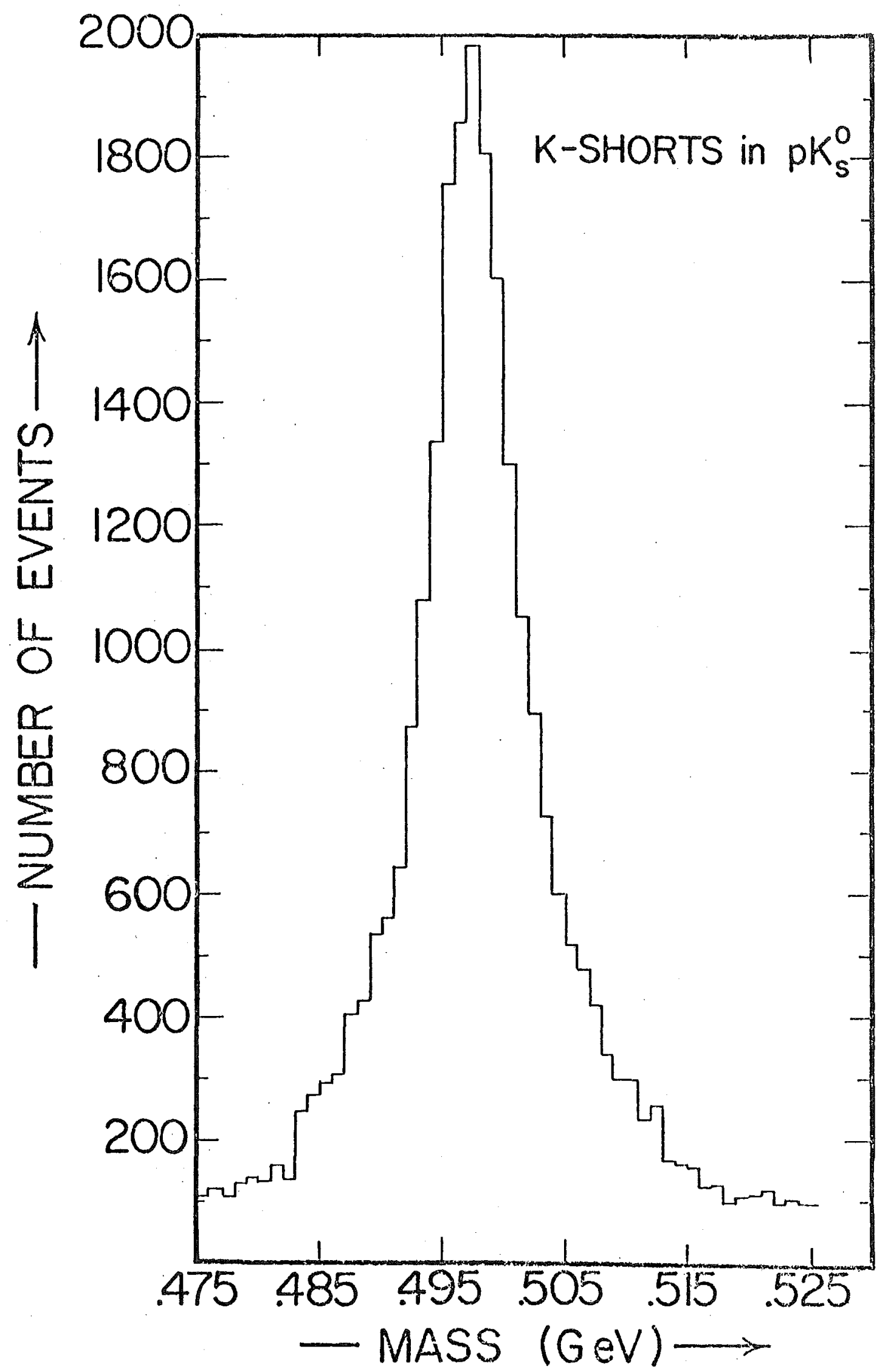

Fig. 29. $\mathrm{K}_{\mathrm{S}}{ }^{\mathrm{O}}$ in $\mathrm{pK}_{\mathrm{S}}^{\mathrm{o}}$ Sample. 
only one $\mathrm{pk}_{\mathrm{S}}{ }^{0}$ entry per event. The state does have two undesirable features. The strangeness-charge correlation is lost since the strangeness of the $\mathrm{K}_{\mathrm{S}}{ }^{\prime} \mathrm{s}$ is indeterminate. In addition, only one out of every three produced $\mathrm{K}^{0}$ 's eventually becomes detectable in the $\pi^{+} \pi^{-}$decay mode of the $\mathrm{K}_{S}{ }^{0}$, effectively lowering the yields into this channel by the same amount. The mass plot for the sum of $\mathrm{pK}_{\mathrm{S}}^{0}$ and its charge conjugate $\left(\overline{\mathrm{p}}_{\mathrm{S}}^{0}\right)$ is shown in Fig. 30. A prominent peak of 55 events is seen at 2.285 $\mathrm{GeV} / \mathrm{c}^{2}$. As anticipated the background is small and the 55 events represent a $6-7 \sigma$ effect. To study the signal in more detail, the plot is broken into baryon and anti-baryon channels (Fig. 31). Approximately equal numbers of events are present in both channels (31 for the baryon versus 24 for the antibaryon). The original plot is also rebinned into $4 \mathrm{MeV} / \mathrm{c}^{2}$ bins (Fig. 32) to extract the mass, width, and number of events. A fit to a gaussian over a locally quadratic background is performed. All parameters are left free. The results of the fit are presented on the same figure. In addition to the statistical error of \pm 1 $\mathrm{MeV} / \mathrm{c}^{2}$ given by the fit a systematic error of $\pm 5 \mathrm{MeV} / \mathrm{c}^{2}$ is included resulting from an uncertainty in the magnet calibrations.

The non-photon induced background in the $\mathrm{pK}_{\mathrm{s}}^{0}$ channel, shown in Fig. 33 (dashed curve) reveals that, excepting the region near $2.285 \mathrm{GeV} / \mathrm{c}^{2}$, the entries are nearly all non-photon in nature. A fit of the $\mathrm{K}_{\mathrm{L}}^{0}$ background, fixing the mass and width at 2.284 $\mathrm{GeV} / \mathrm{c}^{2}$ and $7.2 \mathrm{MeV} / \mathrm{c}^{2}$, respectively, indicates that less than 5 events are not photon induced. The product of the branching 


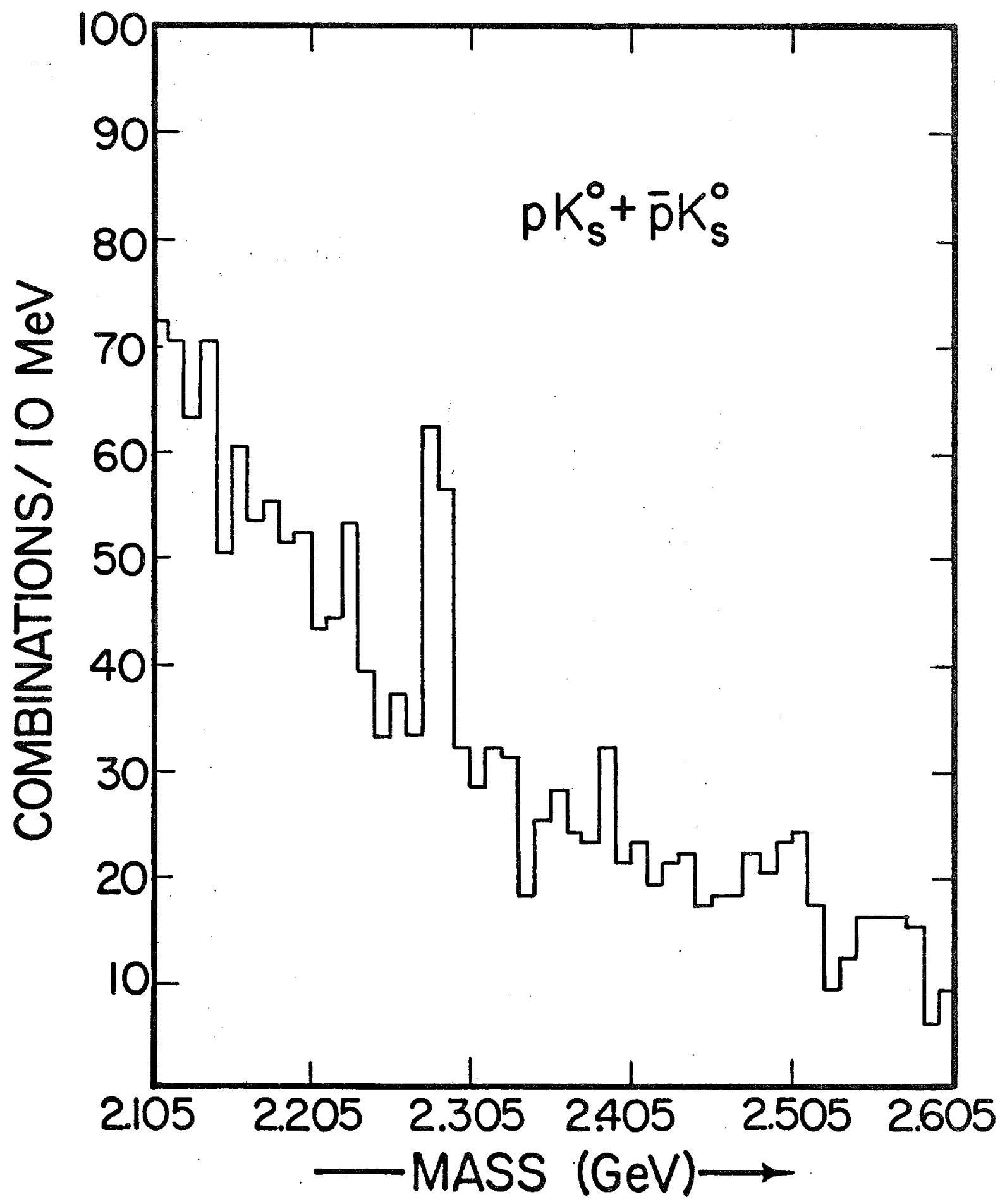

Fig. 30. Mass Distribution of $\mathrm{pK}_{\mathrm{s}}^{\circ}+\overline{\mathrm{pK}}_{\mathrm{s}}^{\circ}$ 


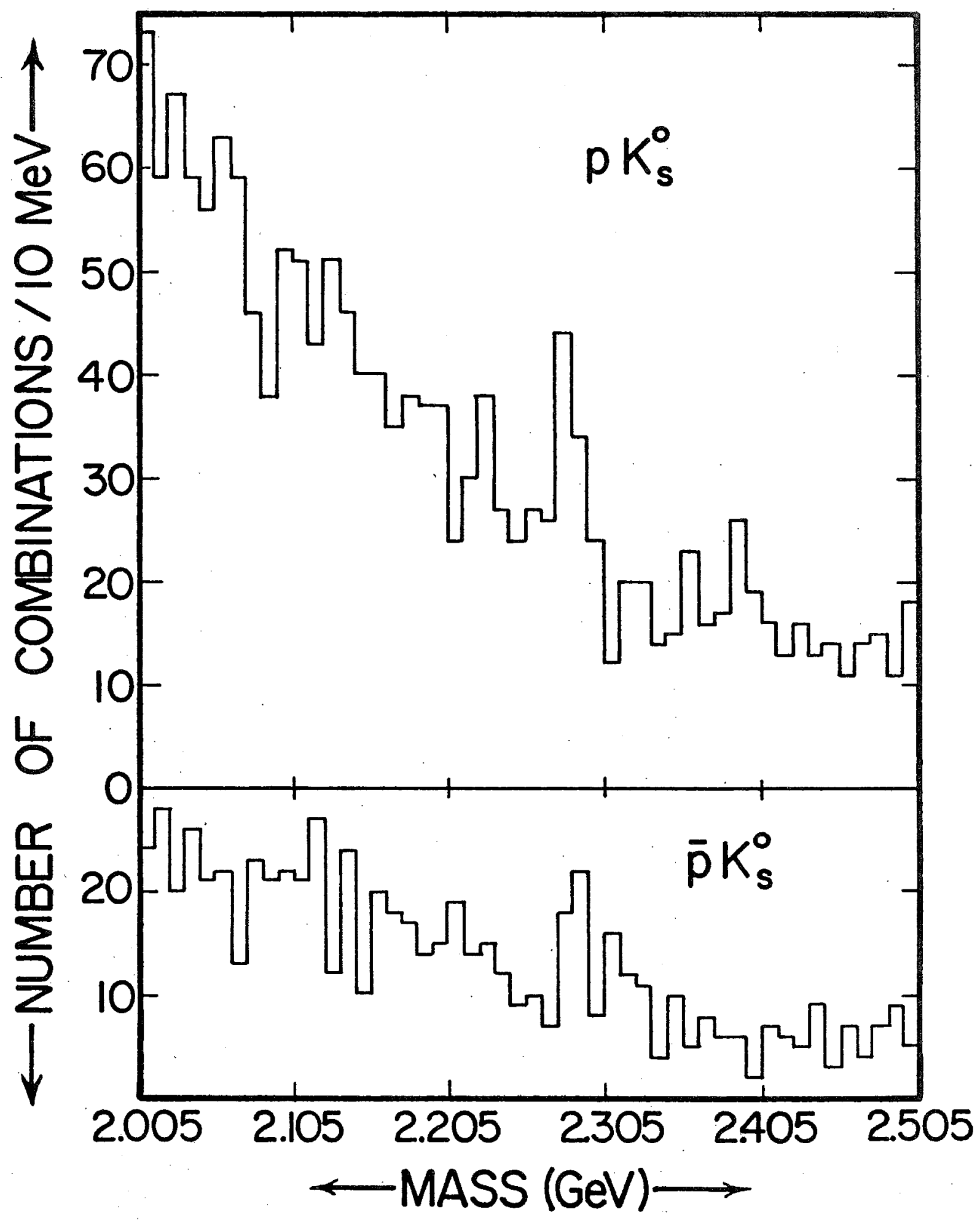

Fig. 31. Mass Distribution of $\mathrm{pK}_{\mathrm{S}}^{\circ}$ (top); $\overline{\mathrm{pK}} \mathrm{s}_{\mathrm{S}}^{\circ}$ (bottom) 


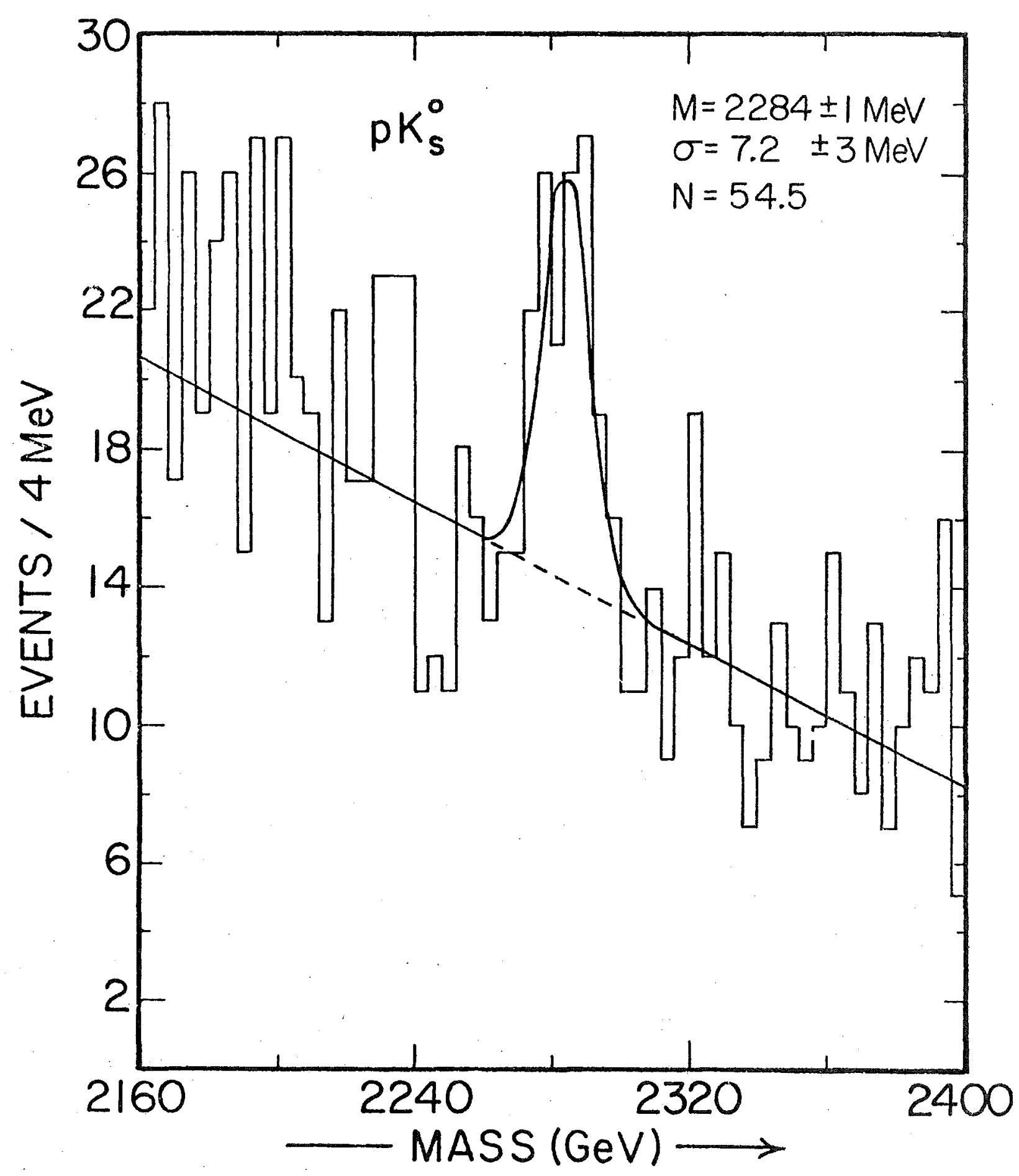

Fig. 32. Fit of $\mathrm{pK}_{\mathrm{S}}{ }^{\circ}$ Mass Distribution. 


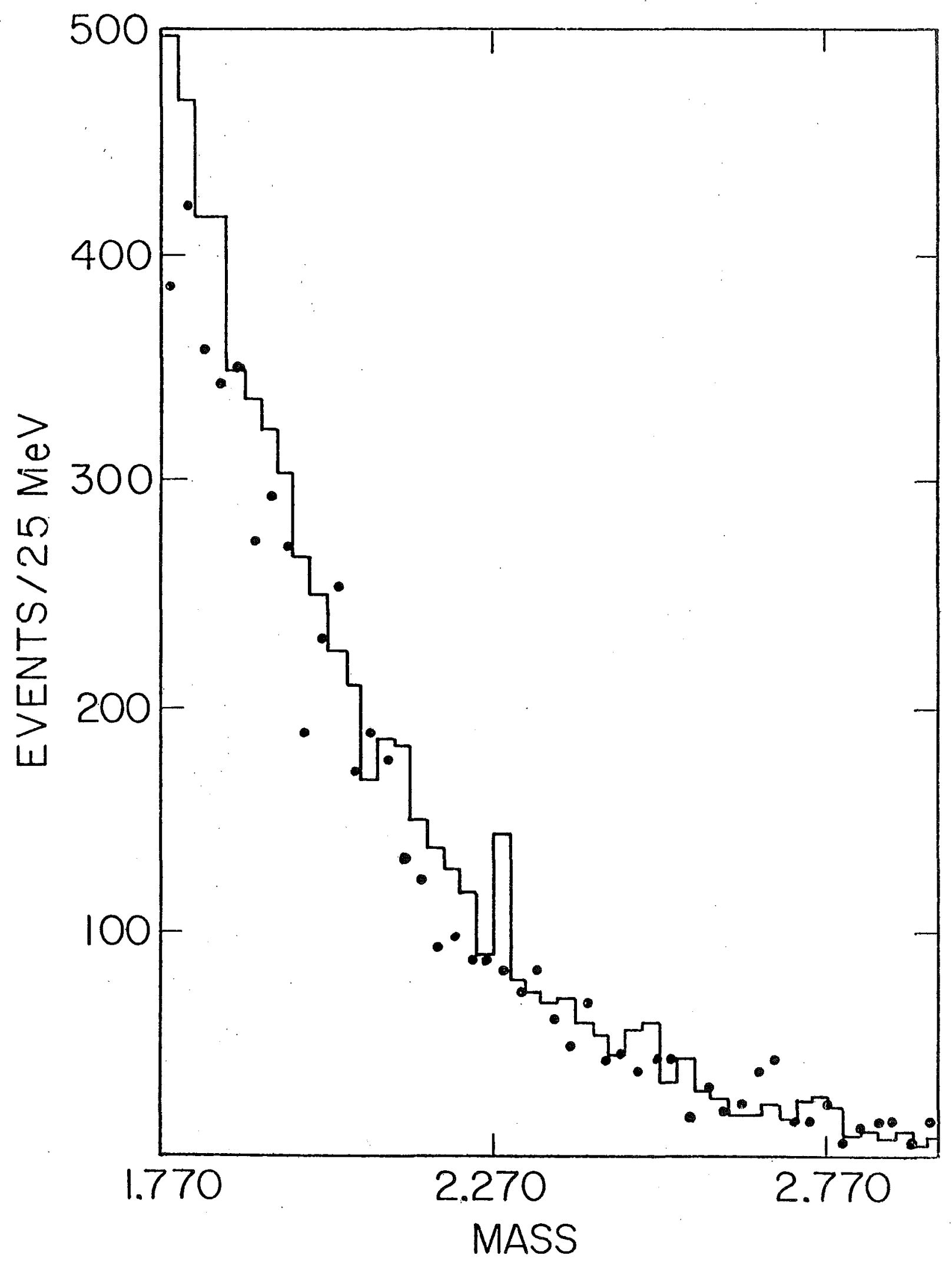

Fig. 33. Mass Distribution of $\mathrm{pK}_{\mathrm{S}}{ }^{\mathrm{o}}$ for All Data (solid); for $\mathrm{K}_{\mathrm{L}}{ }^{\mathrm{O}}$ Data (dots). 
ratio times cross section ( $\sigma B$ ) for this channel based on the observed signal is given in Section $\mathrm{E}$.

A search for the cascade process $\Sigma_{c} \rightarrow \Lambda_{c}{ }^{+} \pi$ was attempted. Looking at the level diagram for the baryons, Fig. A1 shows that there are two $\Sigma_{c}{ }^{\prime} s$, a $J^{P}=1 / 2^{+}$and a $J^{P}=3 / 2^{+}$. One also notes they are a displaced isotriplet (i.e., charges $\pm 2, \pm 1,0$ ). The mass plot for all $\mathrm{pK}_{\mathrm{s}}^{0} \pi$ combinations is shown in Fig. 34. The smaller insert shows the same plot when the $\mathrm{pK}_{\mathrm{S}}^{0}$ is within \pm 10 $\mathrm{MeV} / \mathrm{c}$ of $2.284 \mathrm{GeV} / \mathrm{c}^{2}$. While there is a suggestion of two peaks (one at $2.50 \mathrm{GeV} / \mathrm{c}^{2}$ and the other $2.60 \mathrm{GeV} / \mathrm{c}^{2}$ ), their levels of significance are less than three standard deviations. Different extrapolations of the background through the region between the peaks could completely eliminate these peaks. An alternate view is that the dip between the "peaks" is the nost significant feature of the plot.

Another approach is to plot the mass difference $\Delta M$ $=\mathrm{M}\left(\mathrm{pK}_{\mathrm{S}}{ }^{0} \pi\right)-\mathrm{M}\left(\mathrm{pK}_{\mathrm{S}}{ }^{0}\right)(\mathrm{Fig} \cdot 35)$. Using the mass difference allows for a comparison of the distributions just above and below the $\Lambda_{\mathrm{c}}$ mass with the signal bin, since its shape should be insensitive to the $\mathrm{pK}_{\mathrm{s}}{ }^{0}$ mass. The resolution in this variable may also be better since the error in measuring the $\mathrm{pK}_{\mathbf{S}}{ }^{0}$ mass cancels. (One must remember that the pion cascade of the $\Sigma_{c}$ to the $\Lambda_{c}$ is a strong process and therefore the $\Sigma_{c}$ will likely have a natural width greater than the experimental resolution leaving little to be gained.) The peak associated with the $2.5 \mathrm{GeV} / \mathrm{c}^{2}$ peak (which should appear at $225 \mathrm{MeV} / \mathrm{c}^{2}$ ) is washed out, altering the shape of the background, and thus significantly reducing any significance 


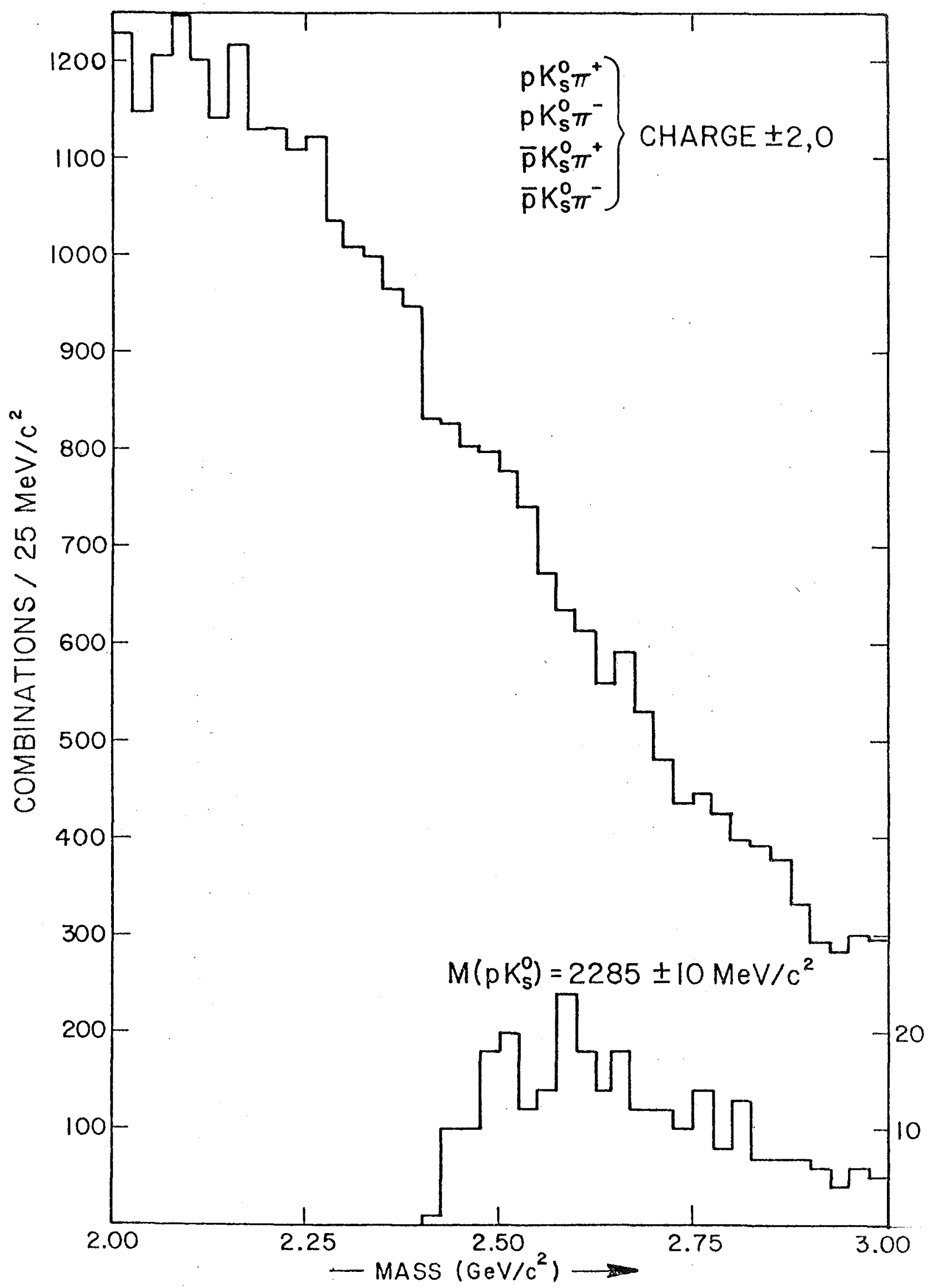

Fig. 34. Mass Distribution of $\mathrm{pK}_{\mathrm{S}}{ }_{\mathrm{S}} \pi$. 


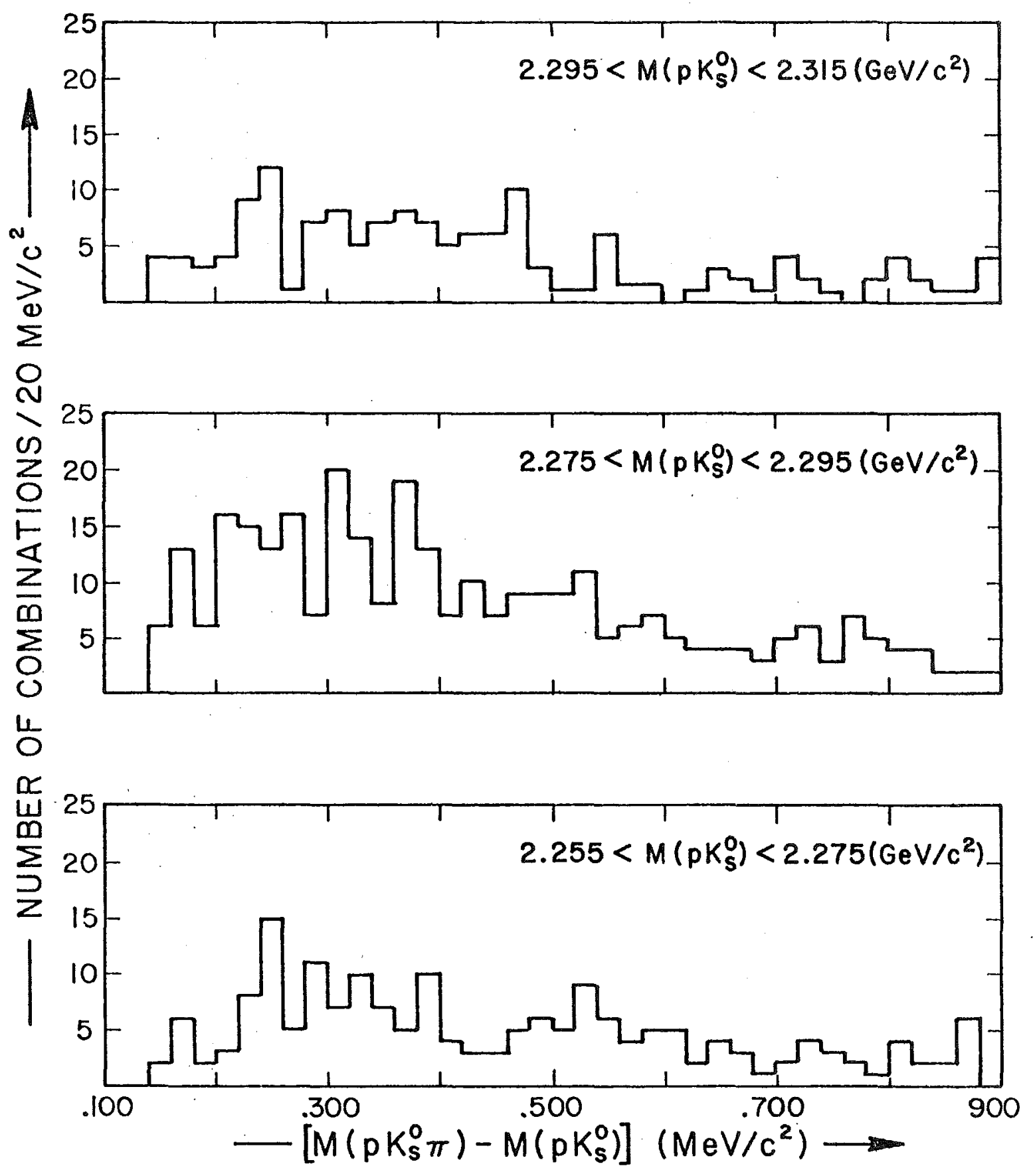

Fig. 35. Cascade Mass Difference for Bins of $\mathrm{pK}_{\mathrm{S}}^{\mathrm{O}}$ Mass. 
one may have been tempted to attach to the second higher mass peak (at $315 \mathrm{MeV} / \mathrm{c}^{2}$. One is forced to conclude that there is no convincing evidence for the cascade process $\Sigma_{c}+\Lambda_{c} \pi \cdot$

D. Monte Carlo

To extract the cross section for the $\mathrm{p} \overline{\mathrm{K}^{0}}$, and the upper limits for the other three states, a Monte Carlo program was developed to simulate the physics, experimental apparatus, and analysis efficiency. The model chosen consists of the photon diffracting off the nucleon target to a c $\overline{\mathrm{c}}$ state of mass $M$ which immediately falls apart to a charmed baryon anti-baryon pair.

In doing the analysis every effort was made to employ only those cuts which could not only be accurately simulated, but which were also model independent. This goal cannot be completely realized since one starts with a built in bias through the apparatus. With only 55 events and a signal to noise of $1: 1$, not much guidance on specific details to incorporate into the model can be found from the data. The model's free parameters which will effect the results are the momentum transferred to the $c \bar{c}$ state along with its mass (M). The precise manner in which the recoiling $\Lambda_{c}^{-}$decays can also effect the result. While the data cannot provide precise details on these quantities, it can place limits on them. The sensitivity of the result can then be explored by varying the model within the confines of these limits while holding the others fixed at their most probable value.

The $t$ distribution of the intermediate $c \bar{c}$ state was taken as $e^{-b t}$. Looking at the observed $t$ distribution for those $\mathrm{pk}_{\mathrm{s}}{ }^{0}$ events within $\pm 10 \mathrm{MeV} / \mathrm{c}$ of $2.284 \mathrm{GeV} / \mathrm{c}^{2} \mathrm{pK}_{\mathrm{s}}{ }^{0}$ peak (Fig. 36a) 
MONTE CARLO COMPARISON

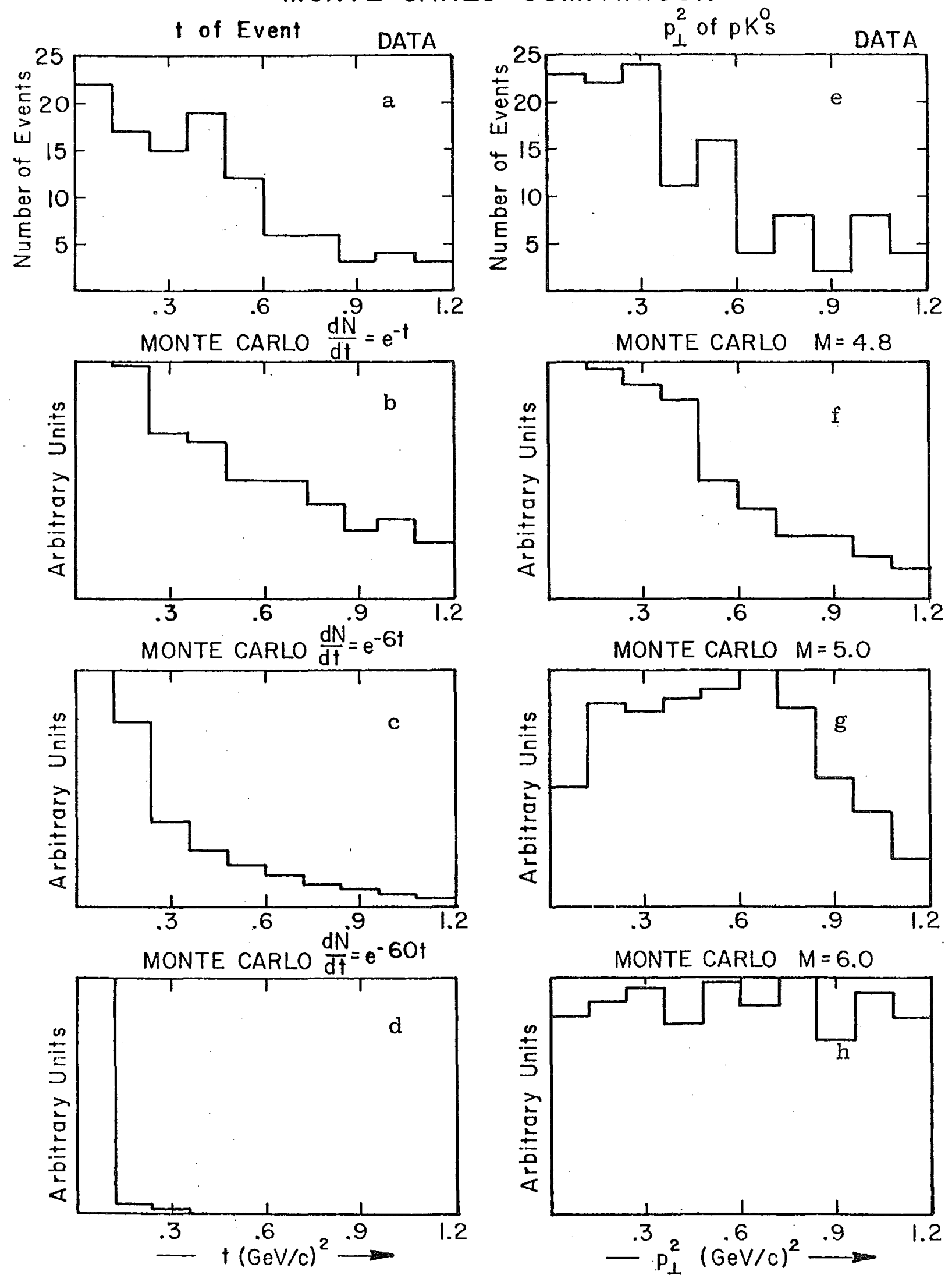

Fig. 36. Monte Carlo Comparison with Data. 
suggests that $b$ is about $1.0(\mathrm{GeV} / \mathrm{c})^{-2}$. Changing the value of $b$ from 1 to 60 produces only a 10\% variation in the acceptance.

Larger variations in the acceptance are produced by altering the mass given the $\bar{c} \overline{\boldsymbol{c}}$ system and the total charged multiplicity generated. The variation of the acceptance on the charged multiplicity enters mainly through the confusion generated in the Cherenkov counters. For the $\Lambda^{0}$ channels this is a small effect (2\%) since they depend on the Cherenkov counters only for the $\Lambda^{0}$ clean-up. The pK $\pi$ state shows a $20 \%$ effect when changing the multiplicity from 5-8 tracks.

The $\mathrm{p}_{\perp}^{2}$ distribution of the $\mathrm{pK}_{\mathrm{S}}^{0}$ signal (Fig. 36a) limits the mass of the $c \bar{c}$ object. The lack of a sharp peak near zero suggests that the mass is not too near threshold. [This peak will be slightly washed out when convoluted with the $p_{\perp}$ received by the parent object, but still cannot generate average value of $0.5(\mathrm{GeV} / \mathrm{C})^{2}$ seen in the data.] The upper limit on the mass is constrained by the same distribution. Any kinetic energy that the $\Lambda_{c^{\Lambda}}^{+}{ }_{c}^{-}$pair may receive when the $\bar{c} \bar{c}$ system disintegrates will be translated to much larger values. Masses larger than 6.0 $\mathrm{GeV} / \mathrm{C}^{2}$ are ruled out unless extra particles are generated to reduce the available energy. For comparison, the $\mathrm{p}_{1}$ distributions of the $\mathrm{pK}_{\mathrm{S}}^{0}$ are shown for $\mathrm{M}=4.8, \mathrm{M}=5.0, \mathrm{M}=6.0 \mathrm{GeV} / \mathrm{c}^{2}$ in Fig. 36f-h. The acceptance is found to vary by $30 \%$ when generating the $c \bar{c}$ system at fixed masses of 4.8 and $6.0 \mathrm{GeV} / \mathrm{c}^{2}$.

The Monte Carlo is written to produce a fake data tape complete with wire hits in the chambers, simulated ADC's, and trigger information. Various detector maladies are included, 
such as chamber inefficiencies, when they can be reliably modeled. The output is then passed through the same sequence of programs as the data. This method has the feature of correctly simulating the geometrical, reconstruction, and analysis efficiencies. The expected experimental width of a generated zero width state is also derived. Table $\mathrm{V}$ summarizes the results.

Various other corrections are included in an ad hoc manner. These are either corrections so simple (target absorption of $2 \%$ per track) that including them in the Monte Carlo is pointless, or so complicated (Cherenkov overefficiency of the central cells) that no reliable model has been found, so that making a reasonable guess based on experience is the only recourse. Table $\mathrm{V}$ includes a compilation of these corrections.

Although other production mechanisms are possible, the fact that roughly equal numbers of baryon and anti-baryons are observed strongly suggests some type of pair production. The charmed mesons also observed in this experiment further support this view, with equal amounts of particle and anti-particle seen. An associated production model would preferentially produce mesons with $\mathrm{C}=-1\left(\overline{\mathrm{D}^{0}}, \mathrm{D}^{-}\right)$and baryons with $\mathrm{C}=+1$ $\left(\Lambda_{\mathrm{c}}{ }^{+}\right)$. The kinematics of this model are such that the meson carries off a large fraction of the photon's energy, while the baryon is produced with a small Feynman $X\left[X=E\left(\Lambda_{c}\right) / E_{\gamma}\right]$. The signal observed has an average $X$ value of 0.55 again supporting a pair production model. 
TABLE V

\section{ACCEPTANCES AND CORRECTIONS}

\begin{tabular}{|c|c|c|c|c|c|c|c|c|}
\hline STATE & $\begin{array}{l}\text { MONTO } \\
\text { CARLO } \\
\text { EFFICIENCY }\end{array}$ & $\begin{array}{r}\text { TARGET } \\
\text { ABSORPT } \\
\end{array}$ & $\begin{array}{l}V^{\circ} \\
\text { BRANCHINC } \\
\text { RATIO }\end{array}$ & $\begin{array}{c}K^{ \pm} \\
\text {DECAY }\end{array}$ & $\begin{array}{c}\check{C} \\
\text { OVER- } \\
\text { EFFICIENCY }\end{array}$ & $\begin{array}{l}\text { JNANALYz } \\
\text { EVENTS }\end{array}$ & $\begin{array}{l}\text { BLE } \\
\prod(\epsilon)\end{array}$ & $\begin{array}{l}\text { RESOLUTION } \\
\text { (FWHRI) }\end{array}$ \\
\hline$\Lambda_{L p \pi}^{0} \pi$ & $13 \%$ & .97 & .64 & 1.0 & .99 & .88 & $7.0 \%$ & $25 \mathrm{MeV} / \mathrm{c}^{2}$ \\
\hline$\Lambda_{L_{p} \pi \pi}^{0} \pi \pi$ & $8.4 \%$ & .94 & .64 & 1.0 & .99 & .88 & $4.4 \%$ & $20 \mathrm{MeV} / \mathrm{c}^{2}$ \\
\hline $\mathrm{pK} \pi$ & $4.5 \%$ & .97 & 1.0 & .95 & .87 & .88 & $3.2 \%$ & $20 \mathrm{MeV} / \mathrm{c}^{2}$ \\
\hline $\begin{array}{l}p \bar{K}_{0}^{0} \\
L_{K}^{0} \\
L_{-\pi^{+}} \pi^{-}\end{array}$ & $5.0 \%$ & .97 & .34 & 1.0 & .93 & .88 & $1.4 \%$ & $20 \mathrm{MeV} / \mathrm{c}^{2}$ \\
\hline
\end{tabular}


E. Cross Sections and Conclusions

The luminosity of the experiment is calculated in Appendix $C$ and found to be $619 \mathrm{nb}^{-1}$. The luminosity, Monte Carlo efficiency, and the corrections listed in Table $V$ can be used to calculate an upper limit on oB for $p K \pi, \Lambda^{0} \pi$, and $\Lambda^{0} \pi \pi \pi$ based on a 30 fluctuation. For the $\mathrm{pK}_{\mathrm{s}}^{0}$ channel where a signal, inconsistent with a fluctuation is observed, the results, of course, represent a determination of the product of the cross section and branching ratio. The $\sigma B$ for the process $\gamma+c \rightarrow \Lambda_{c}^{+}+X$ includes a factor of $1 / 2$, since in a pair production model there are two potentially detectable charmed baryons.

$\begin{array}{cc}\text { State } & \frac{\sigma B}{\Lambda^{0} \pi} \\ \Lambda^{0} \pi \pi \pi & \leqslant 0.9 \mathrm{nb} / \text { nucleon }(90 \% \mathrm{c.1.}) \\ \mathrm{p} \frac{\mathrm{K} \pi}{\mathrm{pK}} & \leqslant 4.0 \mathrm{nb} / \mathrm{nucleon}(90 \% \mathrm{c.1} .) \\ \mathrm{K}^{0} & 3.2 \pm 0.7 \mathrm{nb} / \text { nucleon }\end{array}$

A systematic error of $-20 \%$ to $+40 \%$ should also be included. The large number of events in the $\mathrm{pK} \pi$ and $\Lambda^{0} \pi \pi \pi$ channels result in decreased sensitivity so that these limits are not enlightening. The comparatively large acceptance (7.0\% with all corrections) and the relatively low background $(750$ events/20 $\left.\mathrm{MeV} / \mathrm{c}^{2}\right)$ indicates the relative branching ratio of ${ }^{+} \Lambda_{\mathrm{c}}^{+}$ $\left.\rightarrow \Lambda^{0} \pi\right) /\left(\Lambda_{\mathrm{c}}^{+} \rightarrow \mathrm{p} \overline{K^{0}}\right)$ is less than $1 / 3$.

Taking a $2 \%$ branching ratio for $\Lambda_{\mathrm{C}}^{+} \rightarrow \mathrm{pK}_{\mathrm{S}}^{0}$, as suggested by the MARK II results,24 yields a cross section of $150 \pm 50$ $\mathrm{nb} / \mathrm{nucleon}$. 


\section{CONCLUSIONS}

This final chapter will survey data from other experiments and place the results reported here in proper perspective. The mass of the charmed baryon and its production cross section is reviewed. Finally, the future of charm spectroscopy and the direction which new charm experiments should take is discussed.

\section{A. Comments on the Mass of the $\Lambda_{c}^{+}$}

The number of reported observations of the $\Lambda_{c}^{+}$has been increasing in the last year. There is little doubt as to its existence, but less well settled are its mass and cross section. Two masses appear in the literature, one at $2260 \mathrm{MeV} / \mathrm{c}^{2}$ and one between 2280 and $2290 \mathrm{MeV} / \mathrm{c}^{2}$. The earliest observations tended to favor the lower mass. Those recently reporting masses around $2260 \mathrm{MeV} / \mathrm{c}^{2}$ are the Lampshade Magnet group (LSM) ${ }^{30}$ and the Split Field Magnet group (SFM) ${ }^{31}$ both at CERN. All but one of the groups. (LSM) reporting a mass at $2260 \mathrm{MeV} / \mathrm{c}^{2}$ also quote errors of $20 \mathrm{MeV} / \mathrm{c}^{2}$. The LSM group reports a mass of $2255 \pm 5$ $\mathrm{MeV} / \mathrm{c}^{2}$ where the small error results from restricting the events to be contained within a well-understood portion of their apparatus. (In recent months this number has been adjusted to $2262 \mathrm{MeV} / \mathrm{c}^{2}$.) The Mark II collaboration at $\operatorname{SLAC}^{24}$ along with the UCLA-Saclay group 2 at the ISR favor the higher mass. The UCLASaclay group observes the $\Lambda_{c}^{+}$in two decay modes $\left(\Lambda^{0} \pi \pi^{+} \pi^{-}\right.$and $\left.\mathrm{pK}^{-}{ }^{+}\right)$with slightly different masses $\left(2280 \pm 10 \mathrm{MeV} / \mathrm{c}^{2}\right.$ for $\Lambda^{0} \pi^{+} \pi^{+} \pi^{-}$and $2290 \pm 10 \mathrm{MeV} / \mathrm{c}^{2}$ for $\left.\mathrm{pK}^{-} \pi^{+}\right)$. Mark II has taken great care in understanding their mass scale and appears well entrenched at $2285 \pm 6 \mathrm{MeV} / \mathrm{c}^{2}$. 
The results reported here are in excellent agreement with the $2285 \mathrm{MeV}^{2}$ mass. It should be pointed out that the $D^{0}$ signal observed in this experiment using the same magnet calibrations is within $1 \sigma$ of the reported mass $\left(1863 \mathrm{MeV} / \mathrm{c}^{2}\right)$ with $1861 \pm 2 \mathrm{MeV} / \mathrm{c}^{2}$, where the error is statistical only.

\section{B. Comments on the Charm Cross Section}

The hadronic cross sections reported by the CERN groups have rather large errors due to model uncertainties. The branching ratio times cross section $(\sigma B)$ for the $\Lambda^{0}{ }_{\pi}{ }_{\pi}+{ }^{-}$decay is reported to be between 0.3 and $3.0 \mu \mathrm{b}$. The $\sigma B$ for the $\mathrm{pK}^{-} \pi^{+}$mode is similarly given as $1.2-6.5 \mu \mathrm{b}$.

The only other direct measurement of cross sections for the photoproduction of charmed states is from the $\Omega^{33}$ spectrometer at CERN. Their total cross section, as determined from the semileptonic decay, is given as $800 \mathrm{nb}$. A recent muon experiment ${ }^{34}$ has reported $700 \mathrm{nb}$ by extrapolating from virtual photons to real photons $\left(q^{2}=0\right)$.

Three charm states have been directly observed in this experiment. The $\Lambda_{c}^{+}$cross section is $150 \mathrm{nb}$. The meson cross section is found to be about $450 \mathrm{nb}$, giving a total observed charm cross section of about $600 \mathrm{nb}$. Two factors will push this number higher. Many charmed particles have yet to be seen, most notably those containing strange quarks. The $\Omega$ group has claimed that the photoproduction cross section for the $F$ is about $300 \mathrm{nb}$, although the data presented thus far is not compelling. The second factor likely to drive the total charm cross section higher is the possibility of other production mechanisms. As the 
energy is increased, new thresholds are likely to be crossed through the opening of more inelastic channels. A final number which could be significantly larger than $0.7-1.0 \mu \mathrm{b}$ is not only probable, but likely.

It is interesting to look back at the initial predictions of $1.0-2.0 \mu \mathrm{b}$ and see they were not that far off. Why was the experimental observation of charm so difficult given the excellent starting point? One not only knew the approximate masses, the decay modes, but also the right cross section. The answer to this question is, of course, different for each experiment. One could sight the low branching ratios to detectable states and larger than anticipated backgrounds as prime offenders; however, the real culprit is the increased complexity of charm experiments, both from a hardware and physics viewpoint. As the experimenters become more comfortable with this new complexity, the results may come as easily as it initially looked.

\section{Comments of the Future of Charm Experiments}

While much has been accomplished in charm spectroscopy, much remains to be done. Certainly high on the experimental shopping list is the convincing observation of a charm particle containing a strange quark. However, the emphasis in future charm experiments is likely to swing from spectroscopy to dynamics. Two different measurements are needed. The measurement of the Cabbibo suppressed decay branching ratios provides a valuable check on the theory. The richest experimental field is in production of dynamics. The next round of charm experiments will 
need to observe charm particles in much greater numbers and with excellent signal to noise in order to be able to distinguish between the various production models currently on the market. 
LIST OF REFERENCES

${ }^{1}$ E. Fermi and C. N. Yang, Phys. Rev. 76, 1739 (1949).

${ }^{2}$ S. Sakata, Prog. Theor. Phys. 16, 686 (1956).

${ }^{3}$ M. Gell-Mann and Y. Ne'eman, The Eightfold Way (Benjamin and Sons, New York, 1964).

4M. Gell-Mann, Phys. Lett. 8, 214 (1964).

${ }^{5}$ V. E. Barnes et al., Phys. Rev。 Lett. 12, 204 (1964).

${ }^{6}$ A. Zichichi, Lepton and Hadron Structure (Academic Press, New York, 1975), Vol. 12 .

${ }^{7}$ S. L. Glashow, J. Iliopoulous, and L. Maiani, Phys. Rev. D2, $1285(1970)$.

8B. Bjorken and S. L. Glashow, Phys. Lett. 11, 255 (1964).

${ }^{9}$ S. Weinberg, Phys. Rev. Lett. 19, 1264 (1967); Phys. Rev. D5, 1412 (1972); Phys. Rev. D5, 1962 (1972).

10 A. S. Ebers and B. Lee, Phys. Reports 9C (1973).

11 J. J. Aubert et al., Phys. Rev. Lett. 33, 1404 (1974).

$12 \mathrm{~J}$. E. Augustin et al., Phys. Rev. Lett. 33, 1406 (1974).

$13 \mathrm{~J}$. E. Augustin et al., Phys. Rev. Lett. 33, 1453 (1974).

${ }^{14}$ C. Zweig, CERN Report No. Th401 (1964), unpublished; CERN Report No. Th412 (1964), unpublished.

${ }^{15}$ S. Okubo, Phys. Lett. 5, 165 (1963).

16 J. Iizuka, Supp. Prog. Theor. Phys. 37-38, 21 (1966).

${ }^{17}$ G. Goldhaber et al., Phys. Rev. Lett. 37, 255 (1976); Phys. Rev. Lett. 37, 569 (1976).

${ }^{18}$ P. Alibran et al., Phys. Lett. 74B, 134 (1978).

19 T. Hansl et al., Phys. Lett. 74B, 139 (1978).

20 P. C. Bosetti et a1., Phys. Lett. 74B, 143 (1978). 
21 E. G. Cazzoli et al., Phys. Rev. Lett. 34, 1125 (1975).

22 B. Knapp et a1., Phys. Rev. Lett. 37, 882 (1976).

23 P. Avery et al., Phys. Rev. Lett. 44, 1309 (1980).

$24 \mathrm{~J}$. Kirkby, Proceedings of the International symposium on Lepton and Photon Interactions at High Energies, Fermi National Accelerator Laboratory, Batavia, Illinois, 1979, p. 107.

25 B. Knapp et al., Phys. Rev. Lett. 34, 1040 (1975).

26 T. Nash et al., Phys. Rev. Lett.36, 1233 (1976).

27 C. Quigg, Lectures on Charmed Particles, Fermi National Accelerator Laboratory Report FERMILAB-Conf-78/37-THY.

28 D. Caldwell et al., Phys. Rev。 Lett.40,1222 (1978)。

${ }^{29} \mathrm{M}$. B. Einhorn, Introduction to $\mathrm{SU}(4)$ and the Properties of Charmed Hadrons, Fermi National Accelerator Laboratory Report FERMILAB-Lecture-75/1-THY/EXP.

30 K. L. Giboni et al., Phys. Lett. 85B, 437 (1979)。

${ }^{31}$ D. Druard et al., Phys. Lett. 85B, 452 (1979).

32 W. Lockman et al., Phys. Lett. 85B, 443 (1979).

${ }^{33} \mathrm{~F}$. Kichard, proceedings of the International symposium on Lepton and Photon Interactions at High Energies, Fermi National Accelerator Laboratory, Batavia, I1linois, 1979, p. 469 .

34 M. Mugge, private communication. 


\section{APPENDIX A}

SU (4) AND HADRON SPECTROSCOPY

In the quark model the hadrons are thought of as combinations of the fundamental representation

$$
[4]=q=\left[\begin{array}{l}
u \\
d \\
s \\
c
\end{array}\right]
$$

of $\mathrm{SU}(4)$. The mesons are a bound state of a quark-antiquark combination and the baryons of three quarks. These combinations, however, are not irreducible. This means there are subsets of these representations which transform only among themselves. In group, theory notation (see Ref. 29) one can decompose the mesons as follows

$$
\bar{q} q=[\overline{4}] \times[4]=[1]+[15] .
$$

A more enlightening procedure is to decompose the SU(4) representation into an $\mathrm{SU}(3)$ triplet and $\mathrm{SU}(3)$ singlet. While any of the four quarks can be taken to be the singlet, clearly the most physically meaningful choice is to take the charm quark. The mesons are then decomposed into irreducible sU(3) multiplets which are tagged by their total charm content. This proceeds as follows. (The subscripts indicate the charm content.)

$$
\begin{aligned}
\mathrm{qq}=[\overline{4}] \times[4] & =\left\{[\bar{T}]_{-1}+[\overline{3}]_{0}\right\} \times\left\{[1]_{1}+[3]_{0}\right\} \\
& =[1]_{0}+[\overline{3}]_{1}+[3]_{-1}+\{[\overline{3}] \times[3]\} 0_{0} .
\end{aligned}
$$

Using the relation

$$
[\overline{3}] \times[3]=[1]+[8] \text {, }
$$


one has

$$
\overline{\mathrm{q} q}=\left\{[1]_{0}+\frac{\left.[8]_{0}\right\}}{\overline{q q}}+\frac{[1]}{\bar{c} c}+\frac{[3]}{\bar{c}_{0}}+\frac{[\overline{3}]}{\bar{q} c} \cdot\right.
$$

The properties of these multiplets are best illustrated in a weight diagram (Fig. A1b).

The same procedure can be followed for the baryons, but because the baryons are composed of three identical fermions the overall wave function must be antisymmetric under quark interchange. This complexity can be handled by resorting to a more complete theory of the strong interactions which postulates a hidden quantum number called color. Each of the four flavors of quarks $(u, d, s, c)$ now is also tagged by one of three colors (red, blue, and green). One now has a theory in which the flavors transform according to $\mathrm{SU}(4)$ and the colors according to SU(3). To keep the color hidden the theory demands all physical particles to be color singlets, implying the color portion of the wave function is antisymmetric.

To understand this more completely it is useful to first consider the case of only three flavors of quarks $(u, d, s)$. The decomposition of the baryons in SU(3) proceeds as follows. (The subscripts here refer to the symmetry under quark interchange: s, symmetric; a, antisymmetric; m, mixed symmetry.)

$$
\begin{aligned}
\mathrm{qqq}=[3] \times[3] \times[3] & =[3] \times\{[\overline{3}]+[6]\} \\
& =[1]_{\mathrm{a}}+[8]_{\mathrm{m}}+[8]_{\mathrm{m}}+[10]_{\mathrm{s}},
\end{aligned}
$$

where the relations 


$$
\begin{aligned}
& {[3] \times[3]=[\overline{3}]+[6]} \\
& {[3] \times[6]=[8]+[10]}
\end{aligned}
$$

have been used.

The singlet is totally antisymmetric and the decuplet is totally symmetric. The two octet representations, however, are of mixed symmetry. Given the fact that the wave function must be symmetric in flavor ( $i t$ is antisymmetric in color) and the fact that the lowest lying baryons should have their quarks in an $\mathrm{L}$ $=0$ state (no relative angular momentum), one is forced to ignore the singlet and use only the symmetric combination of the two mixed symmetry octets. [Formally this is done by incorporating the two spin degrees of freedom at the outset, thus expanding the group from SU(3) to SU(6). The [56] representation of SU(6) breaks into two octets of spin $1 / 2$ baryons and four decuplets of $\operatorname{spin} 3 / 2$.

Armed with this knowledge, one can now decompose the baryons according to SU(4).

$$
\begin{aligned}
\mathrm{qqq} & =[4] \times[4] \times[4] \\
& =[4]_{\mathrm{a}}+[20]_{\mathrm{m}}+[20]_{\mathrm{m}}+[20]_{\mathrm{s}}
\end{aligned}
$$

The situation here is similar to the $\mathrm{SU}(3)$ case. There is one totally antisymmetric, one totally symmetric, and two mixedsymmetry representations. The lowest lying baryons come from the totally symmetric [20] representation and the symmetric combination of the two mixed-symmetry [20] representations. [Again to be formally correct one must incorporate spin from the start, expanding $\mathrm{SU}(4)$ to $\mathrm{SU}(8) \cdot$ ] 
As in the case of the mesons it is useful to break these SU(4) representations down by charm content.

$$
\begin{aligned}
& {[20](\operatorname{spin} 1 / 2)=\quad[3]_{2}+[\overline{3}]_{1}+[6]_{1}+[8]_{0}} \\
& {[20](\operatorname{spin} 3 / 2)=[1]_{3}+[3]_{2}+[6]_{1}+[10]_{0} .}
\end{aligned}
$$

The weight diagrams for these two representations are shown in Fig. A1c,d. 
CHARM SPECTROSCOPY

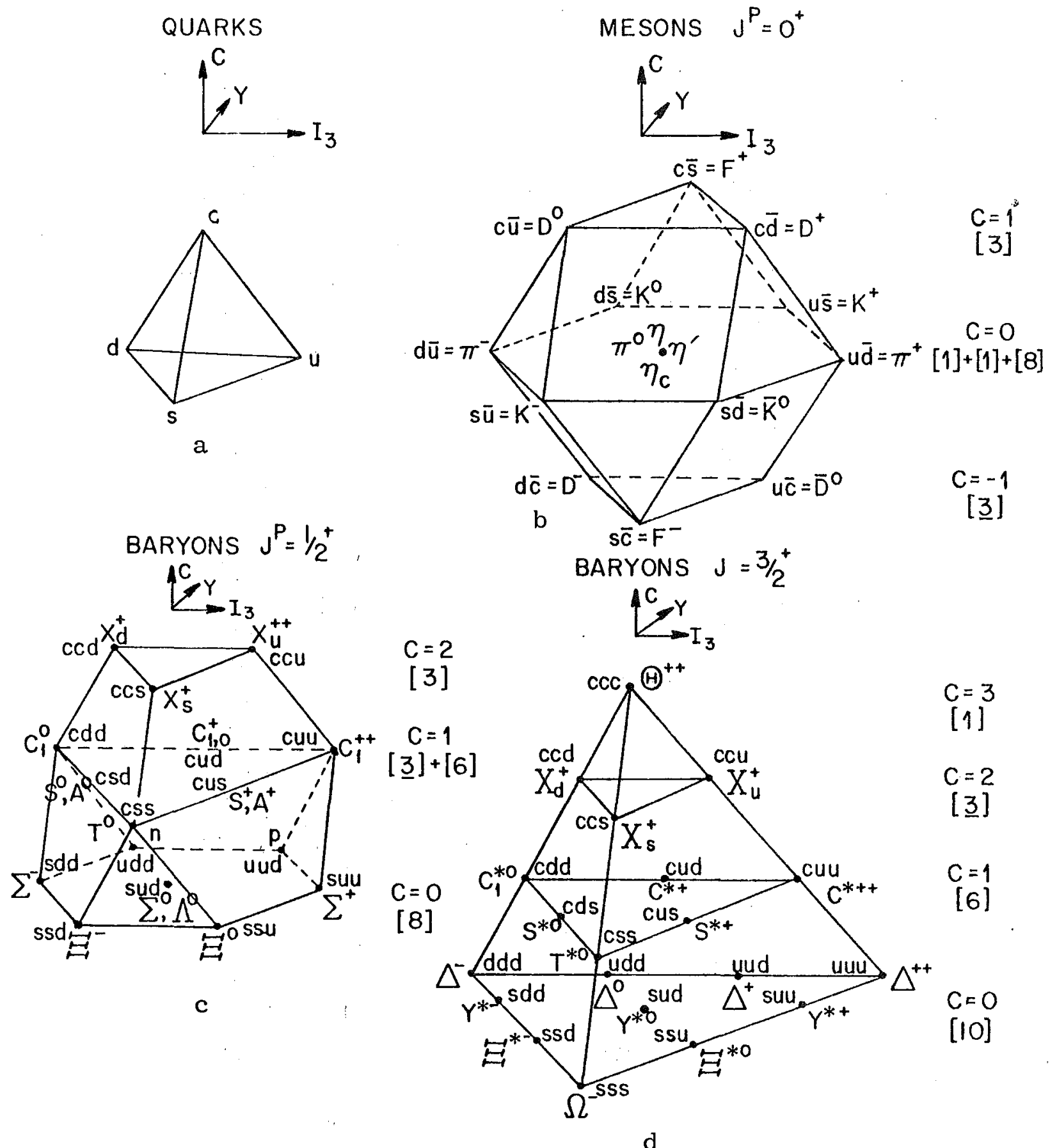

Fig. A 1. Weight Diagrams of Quarks (a); Mesons (b); Baryons (c,d). 
APPENDIX B

NEUTRAL STRANGENESS CHANGING WEAK CURRENTS

In this appendix we show, by proceeding in direct analogy with the weak leptonic current, how one is naturally led to the charm model. The charged weak hadronic current is experimentally observed to have the form

$$
\mathrm{J}_{\mathrm{W}}^{\mathrm{c}}=\overline{\mathrm{u}} \cos \theta \mathrm{d}+\overline{\mathrm{u}} \sin \theta \mathrm{s}+\text { hermtian conjugate. }
$$

If one identifies

$$
d_{\theta}=\cos \theta d+\sin \theta s,
$$

where $\theta$ is the Cabbibo angle, the charged hadron weak current has the same flavor structure as its leptonic counterpart, namely

$$
\mathrm{J}_{\mathrm{W}}=\overline{\mathrm{q}}{ }^{\mathrm{q}} \mathrm{q}+\overline{\mathrm{q} \tau}{ }^{-} \mathrm{q} \text {, }
$$

where $\tau^{ \pm}$is the charge raising (lowering) operator. The $q^{\prime} s$ represent

$$
\frac{\text { Lepton }}{\left[\begin{array}{l}
\mathrm{e} \\
v \\
\mathrm{e}
\end{array}\right]} \quad \frac{\text { Hadron (quark) }}{\left[\begin{array}{l}
\mathrm{u} \\
\mathrm{d}_{\theta}
\end{array}\right]}
$$

The weak neutral hadronic current is given by

$$
\mathrm{J}_{\mathrm{W}}^{0}=1 / 2\left[\mathrm{~J}_{\mathrm{W}}^{+}, \mathrm{J}_{\mathrm{W}}^{-}\right]=1 / 2 \overline{\mathrm{q}}\left[\tau^{+}, \tau^{-}\right] \mathrm{q}=\overline{\mathrm{q} \tau}{ }_{3} \mathrm{q} \cdot
$$

Writing this out explicitly

$$
\begin{aligned}
\mathrm{J}_{\mathrm{W}}^{0}=\left[\overline{\mathrm{u}}, \overline{\mathrm{d}}_{\theta}\right]\left[\begin{array}{rr}
1 & 0 \\
0 & -1
\end{array}\right]\left[\begin{array}{l}
\mathrm{u} \\
\mathrm{d}_{\theta}
\end{array}\right] & =\left(\overline{\mathrm{u}} \mathrm{u}-\overline{\mathrm{d}}_{\theta} \mathrm{d}_{\theta}\right) \\
& =\left(\overline{\mathrm{u}} \mathrm{u}-\overline{\mathrm{d} d} \cos ^{2} \theta-\overline{\mathrm{s} s} \sin ^{2} \theta\right) \\
& -(\overline{\mathrm{s}} \mathrm{d}+\overline{\mathrm{d}} \mathrm{s}) \cos \theta \sin \theta
\end{aligned}
$$


The first piece corresponds to a strangeness conserving portion. The second piece results in a strangeness changing neutral current which is not experimentally observed. One can continue the analogy with the leptons and introduce a second set of quarks corresponding to the muon doublet.

$\frac{\text { Lepton }}{\left[\begin{array}{l}\mu \\ \nu_{\mu}\end{array}\right]} \frac{\text { Hadron (quark) }}{\left[\begin{array}{l}c \\ s_{\theta}\end{array}\right],}$

where $s_{\theta}=s \cos \theta-d \sin \theta$. Writing out the neutral current for this set

$$
\begin{aligned}
\mathrm{J}_{W^{\prime}}^{0}=\left[\begin{array}{c}
\bar{c}, \bar{s} \\
{ }_{\theta}
\end{array}\right]\left[\begin{array}{rr}
1 & 0 \\
0 & -1
\end{array}\right]\left[\begin{array}{l}
c \\
s_{\theta}
\end{array}\right] & =\left(\bar{c} c-\bar{s}_{\theta} s_{\theta}\right) \\
& =\left(\overline{c c}-\bar{s} s \cos ^{2} \theta-\overline{d d} \sin ^{2} \theta\right) \\
& +(\overline{s d}+\bar{d} s) \cos \theta \sin \theta .
\end{aligned}
$$

Adding $J_{W}^{0}+J_{W}^{0}$, to get the total neutral weak hadronic current eliminates the objectionable strangeness changing current, giving

$$
J_{W}^{0}=(\bar{c} c+\bar{u} u-\bar{d} d-\bar{s} s) .
$$

\section{CHARM PARTICLE DECAY}

Since charm is conserved by the strong interactions, the decay of charm particles proceeds by the weak force. From the above one sees that the neutral weak current does not play a role since there are no couplings between quarks of different flavors (i.e., the weak neutral hadronic current is flavor conserving). The decay of charm particles, therefore, is completely governed 
by the charged weak current in direct analogy with muon decay. The weak Hamiltonian (ignoring coupling constants and spatial dependence) for the leptons is

$$
\begin{aligned}
H & =J^{+} J^{-}+\text {hermtian conjugate } \\
& =\left(\bar{\nu}_{e} e+\bar{\nu}_{\mu} \mu\right)\left(\overline{\mathrm{e} \nu} \mathrm{e}+\bar{\mu} \nu_{\mu}\right) \\
& =\bar{\nu}_{\mathrm{e}} \mathrm{e}^{\overline{\mathrm{e} \nu}} \mathrm{e}+\bar{\nu}_{\mu} \mu \overline{\mathrm{e} \nu} \mathrm{e}+\bar{\nu}_{\mathrm{e}} \mathrm{e}^{\bar{\mu} \nu_{\mu}}+\bar{\nu}_{\mu} \mu \bar{\mu} \nu_{\mu} .
\end{aligned}
$$

The first and last terms describe elastic neutrino scattering from electrons and muons. The middle terms are responsible for the decay

$$
\mu^{-} \rightarrow \mathrm{e}+\bar{\nu}_{\mathrm{e}}+\nu_{\mu}
$$

The weak decay of the charm quark is described by the same term once the following substitutions are made.

$$
\left[\begin{array}{l}
\mathrm{e} \\
\nu_{\mathrm{e}}
\end{array}\right]+\left[\begin{array}{l}
\mathrm{u} \\
d_{\theta}
\end{array}\right] \cdot\left[\begin{array}{l}
\mu \\
\nu_{\mu}
\end{array}\right]+\left[\begin{array}{l}
\mathrm{c} \\
\mathrm{s}_{\theta}
\end{array}\right] \cdot
$$

The term then becomes

$$
\begin{aligned}
& \bar{\nu}_{e} e^{\bar{\mu} \nu_{\mu}}+\bar{d}_{\theta} u \bar{c} s_{\theta} \\
& =(\bar{d} \cos \theta+\bar{s} \sin \theta) u \bar{c}(s \sin \theta-d \sin \theta) \\
& =\overline{\mathrm{d}} \mathrm{u} \overline{\mathrm{c}} \mathrm{s} \cos ^{2} \theta \\
& \text { - } \bar{d} \text { u } \bar{c} d \sin \theta \cos \theta \quad(c+u d \bar{d}) \\
& +\frac{\bar{s}}{\mathrm{u}} \overline{\mathrm{c}} \mathrm{s} \sin \theta \cos \theta \quad(c+\mathrm{u} s \overline{\mathrm{s}}) \\
& -\bar{s} u \bar{c} d \sin ^{2} \theta \quad(c+u d s) \text {. }
\end{aligned}
$$

The terms are arranged in decreasing order of strength since sin $\theta$ $=0.25, \cos \theta=0.98$. The Cabbibo favored decay of the charm quark is to the strange quark and thus obeys the selection rule

$$
\Delta Q=\Delta S=\Delta C=1 \text {. }
$$


Taking the $\Lambda_{c}^{+}$as an example, one can write down a sampling of Cabbibo favored decays.

$$
\begin{aligned}
\Lambda_{\mathrm{c}} & +\Lambda^{0} \pi^{+} \\
& \rightarrow \mathrm{pK}^{-} \pi^{+} \\
& \rightarrow \mathrm{p}_{0} .
\end{aligned}
$$

One can add arbitrary numbers of $\pi^{\prime} \mathrm{s}$ to these modes (either $\pi^{0}$ 's or $\pi^{+} \pi^{-}$pairs) provided, of course, the decay is kinematically allowed. Thus one can have

$$
\begin{aligned}
\Lambda_{C} & \rightarrow \Lambda^{0} \pi \pi^{+} 0 \\
& \rightarrow \Lambda^{0} \pi{ }^{+}{ }^{+}- \\
& \rightarrow \mathrm{p} \overline{\mathrm{K}}_{0} \pi^{0} \\
& +\mathrm{p} \overline{\mathrm{K}}_{0}^{+} \pi^{+}-
\end{aligned}
$$




\section{APPENDIX C}

\section{CROSS SECTION AND LUMINOSITY DETERMINATION}

The cross section for a process is given by

$$
\sigma=\mathrm{N}_{\mathrm{EVTS}} /\left(\mathrm{N}_{\gamma} \mathrm{N}_{S C}{ }^{\mathrm{B}} \varepsilon\right)
$$

where $\mathrm{N}_{\text {EVTS }}=$ Number of events observed;

$\mathrm{N}_{\gamma} \quad=$ Number of incident photons;

$\mathrm{N}_{\mathrm{SC}}=$ Number of scattering centers in the target;

B = Branching ratio into this final state;

$\varepsilon=$ Experimental detection efficiency.

A useful quantity to define is the luminosity.

$$
\mathcal{L}=\mathrm{N}_{\gamma} \mathrm{N}_{\mathrm{SC}} \text {, }
$$

since it does not depend on a particular process.

The number of photons is determined from the total power collected in the quantometer. To obtain this number one needs to know the spectrum shape.

$$
\mathrm{dN} / \mathrm{dE}=\mathrm{N}_{\gamma} \mathrm{f}(\mathrm{E})
$$

where $\int f\left(E_{\gamma}\right) d E_{\gamma}=1$, so that $N_{\gamma}$ is the total number of photons. The shape of the spectrum was measured by placing a lead glass block in the photon beam. Analysis of the pulse height spectrum gives the curve shown in Fig. 1 .

Since the quantameter measures the total power in the beam, one needs to perform the integral

$$
\begin{aligned}
P=C \int E_{\gamma} d N / d E_{\gamma} d_{\gamma} & =C N_{\gamma} \int f\left(E_{\gamma}\right) d E_{\gamma} \\
& =C N_{\gamma}\left\langle E_{\gamma}\right\rangle .
\end{aligned}
$$


$\mathrm{C}$ is a calibration constant determined by placing the quantameter in a $90 \mathrm{GeV} / \mathrm{c}$ electron beam. $\mathrm{C}$ is found to be $8510 \mathrm{ion} / \mathrm{GeV}$. The number of photons is therefore

$$
N=(P / C) /\left\langle E_{\gamma}\right\rangle
$$

The total power (P) collected was $6.84 \times 10^{13} \mathrm{GeV}$ and the average energy $\left(\left\langle E_{\gamma}\right\rangle\right)$ is $42.54 \mathrm{GeV}$, so this means that the total number of photons is

$$
\mathrm{N}=1.61 \times 10^{12}
$$

A number of corrections must be made to this raw number. Since the Monte Carlo acceptances are computed only for photons with energies greater than $50 \mathrm{GeV}$ and a high energy cut of 200 $\mathrm{GeV}$ is placed on the data, only the photons in that energy range $(50-200 \mathrm{GeV})$ are used.

$$
\begin{aligned}
\mathrm{N}_{\gamma} & =\mathrm{N}_{\gamma} \int_{50}^{200} \mathrm{f}\left(\mathrm{E}_{\gamma}\right) \mathrm{dE}_{\gamma} \\
& =0.304 * \mathrm{~N}_{\gamma} \\
& =4.89 \times 10^{11} .
\end{aligned}
$$

Two deadtime corrections are also required, the master gate deadtime of $13 \%$ and the slow logic deadtime of $11.5 \%$. The total number of "live" photons is

$$
\mathrm{N}=3.77 \times 10^{11}
$$

The number of scattering centers per $\mathrm{cm}^{2}$ is given by

$$
\mathrm{N}_{\mathrm{SC}}=\rho 1 \mathrm{~A},
$$


where $\rho=$ density of target

$1=$ length of target

$A=$ Avogadro's number.

The target is $2.12 \mathrm{~cm}$ of scintillator $\left(\rho=1.05 \mathrm{gm} / \mathrm{cm}^{3}\right)$ and 0.15 $\mathrm{cm}$ of aluminum $\left(\rho=2.7 \mathrm{gm} / \mathrm{cm}^{3}\right)$. The number of scattering centers is

$$
\mathrm{N}_{\mathrm{SC}}=1.58 \times 10^{23} / \mathrm{cm}^{2}
$$

Multiplying the number of photons by the number of scattering centers gives the luminosity

$$
\mathrm{L}=619 \mathrm{nb}^{-1} \text {. }
$$




\section{VITA}

\section{James John Russell}

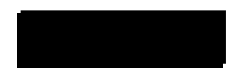

He entered Ohio State University in 1968 and was graduated with a Bachelor of Science degree in 1972. Since 1972 he has held teaching and research assistantships in the Physics Department of the University of Illinois at Urbana-Champaign. In 1973 he received a Master of Science degree from the University of Illinois at Urbana-Champaign. 